University of Tennessee Health Science Center

UTHSC Digital Commons

$12-2006$

\title{
Tyrosine Phosphorylation of Villin: Effects on Actin Dynamics, Cell Morphology and Cell Migration
}

\author{
Alok Tomar \\ University of Tennessee Health Science Center
}

Follow this and additional works at: https://dc.uthsc.edu/dissertations

Part of the Amino Acids, Peptides, and Proteins Commons, and the Medical Cell Biology Commons

\section{Recommended Citation}

Tomar, Alok, "Tyrosine Phosphorylation of Villin: Effects on Actin Dynamics, Cell Morphology and Cell Migration" (2006). Theses and Dissertations (ETD). Paper 271. http://dx.doi.org/10.21007/ etd.cghs.2006.0322.

This Dissertation is brought to you for free and open access by the College of Graduate Health Sciences at UTHSC Digital Commons. It has been accepted for inclusion in Theses and Dissertations (ETD) by an authorized administrator of UTHSC Digital Commons. For more information, please contact jwelch30@uthsc.edu. 


\title{
Tyrosine Phosphorylation of Villin: Effects on Actin Dynamics, Cell Morphology and Cell Migration
}

\author{
Abstract \\ Cell migration is a key aspect of many normal and abnormal biological processes, including embryonic \\ development, defense against infections, wound healing, and tumor cell metastasis. In this study we \\ demonstrate that an epithelial cell actin-binding protein, villin, plays a crucial role in the process of cell \\ migration. Overexpression of villin in doxycyline-regulated HeLa Tet-off and MDCK Tet-off cells enhanced \\ cell migration. We further demonstrate that tyrosine phosphorylation of villin by c-src is required for villin- \\ induced cell migration. Previously, we identified four tyrosine phosphorylation sites in the amino-terminal \\ domain of villin. I further identified six new sites in the carboxylterminal region of the villin core. \\ Collectively we have now documented all phosphorylatable tyrosine residues in villin and mapped them to \\ villin's functions. To further investigate the role of tyrosine phosphorylation sites in cell migration, we \\ used phosphorylation site mutants (tyrosine to phenylalanine or tyrosine to glutamic acid) stably \\ transfected in HeLa and MDCK Tet-off cells. We determined that tyrosine phosphorylation at amino- \\ terminus of villin played an essential role in cell migration as well as in the reorganization of the actin \\ cytoskeleton. The carboxyl-terminal phosphorylation sites were found to be critical for villin's interaction \\ with its ligand PLC-and for its localization to the developing lamellipodia in a motile cell. Collectively, \\ these studies define how biophysical events such as cell migration are actuated by biochemical signaling \\ pathways involving tyrosine phosphorylation of actin binding proteins, in this case villin.

\section{Document Type} \\ Dissertation

\section{Degree Name} \\ Doctor of Philosophy (PhD)

\section{Program} \\ Interdisciplinary Program \\ Research Advisor \\ Seema Khurana, Ph.D.

\section{Keywords} \\ actin, villin, cell migration, tyrosine phosphorylation, wound healing, phosphomimetics, lamellipodia, EGF, \\ PLC, PIP2

\section{Subject Categories} \\ Amino Acids, Peptides, and Proteins | Chemicals and Drugs | Medical Cell Biology | Medical Sciences | \\ Medicine and Health Sciences
}




\title{
Tyrosine phosphorylation of villin: Effects on actin dynamics, cell morphology and cell migration
}

\author{
A Dissertation \\ Presented for \\ The Graduate Studies Council \\ The University of Tennessee \\ Health Science Center
}

\author{
In Partial Fulfillment \\ Of the Requirements for the Degree \\ Doctor of Philosophy \\ In the Interdisciplinary Program \\ Of Graduate Health Science \\ From The University of Tennessee
}

By
Alok Tomar
December, 2006 
Chapter 2 and Appendix A @ 2004 by The American Society for Cell Biology

Chapter 3 and Appendix B (c) 2006 by The American Society for Biochemistry and Molecular Biology 


\section{Dedication}

This dissertation is dedicated to my parents,

N.S. Tomar and Bhagvati Tomar,

my sister Archana, my brother Sandeep

and to my wife, Pratibha

for their love and support 


\section{Acknowledgements}

I would like to thank my advisor, Dr. Seema Khurana for investing a great deal of time and effort on my project and for her continuous and much needed encouragement and guidance. I would also like to express my gratitude to other committee members, Dr. Christopher Waters, Dr. Kafait U Malik, Dr. Radhakrishna Rao and Dr. Jie Zheng for their great advice based on years of experience. I would also like to thank the American society for cell biology and the American society for biochemistry and molecular biology for granting me the permission to use my published articles for this dissertation.

I am also grateful to my colleagues, Dr. Narendra Kumar, Dr. Yaohong Wang, and Dr. Sudeep George, for their critical comments, and helpful discussion of the results and problems I encountered in the lab. I am also thankful to my parents N. S. Tomar and Bhagvati Tomar for motivating me towards my goals. I would specially like to thank my wife for her patience and endless support. 


\section{Abstract}

Cell migration is a key aspect of many normal and abnormal biological processes, including embryonic development, defense against infections, wound healing, and tumor cell metastasis. In this study we demonstrate that an epithelial cell actin-binding protein, villin, plays a crucial role in the process of cell migration. Overexpression of villin in doxycyline-regulated HeLa Tet-off and MDCK Tet-off cells enhanced cell migration. We further demonstrate that tyrosine phosphorylation of villin by c-src is required for villin-induced cell migration. Previously, we identified four tyrosine phosphorylation sites in the amino-terminal domain of villin. I further identified six new sites in the carboxylterminal region of the villin core. Collectively we have now documented all phosphorylatable tyrosine residues in villin and mapped them to villin's functions. To further investigate the role of tyrosine phosphorylation sites in cell migration, we used phosphorylation site mutants (tyrosine to phenylalanine or tyrosine to glutamic acid) stably transfected in HeLa and MDCK Tet-off cells. We determined that tyrosine phosphorylation at amino-terminus of villin played an essential role in cell migration as well as in the reorganization of the actin cytoskeleton. The carboxyl-terminal phosphorylation sites were found to be critical for villin's interaction with its ligand PLC- $\gamma_{1}$ and for its localization to the developing lamellipodia in a motile cell. Collectively, these studies define how biophysical events such as cell migration are actuated by biochemical signaling pathways involving tyrosine phosphorylation of actin binding proteins, in this case villin. 


\section{Table of Contents}

$\begin{array}{ll}\text { Chapter 1. Introduction } & 1\end{array}$

1.1 The actin cytoskeleton 2

1.2 Villin: An actin binding protein 6

1.2.1 $\mathrm{PIP}_{2}$ regulates actin-severing, -capping and -crosslinking activity of villin $\quad 7$

1.2.2 Calcium regulates villin's actin-severing and -capping activity

1.2.3 Tyrosine phosphorylation of villin regulates actin-severing, -nucleation and -bundling activities of villin

1.3 Cell migration

1.3.1 Role of Rho family GTPases in lamellipodia formation

1.3.2 Role of actin in lamellipodia formation

1.3.3 Role of actin binding proteins in lamellipodia formation

Chapter 2. Regulation of cell motility by tyrosine phosphorylated villin

2.1 Introduction

2.2 Materials

2.3.1 Villin cDNA construct

2.3.2 Phosphorylation site mutants of villin

2.3.3 Preparation of recombinant adenovirus expressing dominant negative c-src kinase

2.3.4 Transfection of Tet-Off HeLa cells with full-length and mutant villin cDNA

2.3.5 Cell motility assay 
$\begin{array}{ll}\text { 2.3.6 Phospho-villin antibodies } & 32\end{array}$

2.3.7 Immunofluorescence microscopy 33

2.3.8 F-actin content measurements in HeLa cells expressing wild-type or mutant villin proteins

2.3.9 Cell proliferation measurement 34

2.4 Results $\quad 35$

2.4.1 Cells expressing villin migrate faster than villin-null cells 35

2.4.2 Tyrosine phosphorylation of villin is essential for villin-induced cell migration $\quad 39$

2.4.3 Tyrosine phosphorylation at $\mathrm{Y}-60, \mathrm{Y}-81$, and $\mathrm{Y}-256$

is essential for villin-induced cell migration

2.5 Discussion

Chapter 3. Interaction of phospholipase $\mathrm{C}-\gamma_{1}$ with villin regulates epithelial cell migration

3.1 Introduction

$\begin{array}{ll}3.2 \text { Materials } & 65\end{array}$

$\begin{array}{ll}\text { 3.3 Methods } & 67\end{array}$

3.3.1 Tyrosine phosphorylation of villin in TKX1 cells 67

3.3.2 Amino-terminal truncation mutants of villin 67

3.3.3 Substitution of tyrosine with phenylalanine in villin truncation mutants

3.3.4 Urea denaturation assay

3.3.5 Measurement of actin-polymerization and -depolymerization by phosphorylated wild-type and point mutants of villin

3.3.6 Measurement of actin uncapping by phosphorylated wildtype and point mutants of villin 
3.3.7 Transfection of HeLa and MDCK Tet-Off cells with full-length and mutant villin cDNA

$\begin{array}{ll}\text { 3.3.8 Cell motility assay } & 73\end{array}$

$\begin{array}{ll}\text { 3.3.9 Immunofluorescence microscopy } & 73\end{array}$

3.3.10 Measurement of lamellipodial protrusion rate 74

3.3.11 Immunoprecipitation and western blot analysis $\quad 75$

3.3.12 Separation of Triton-soluble and -insoluble pool 75

3.3.13 Identification of apoptotic cells using HOECHST 33258 $\begin{array}{ll}\text { staining } & 76\end{array}$

$\begin{array}{ll}3.4 \text { Results } & 76\end{array}$

3.4.1 The carboxy-terminus of villin is tyrosine phosphorylated $\quad 76$

3.4.2 Identification of one major and two minor phosphorylation sites in CT2 $\quad 80$

3.4.3 Identification of two major phosphorylation sites in villin truncation mutant CT3

3.4.4 Y461 is the major phosphorylation site in the villin truncation mutant CT4

3.4.5 Functional significance of tyrosine phosphorylation sites identified in the carboxy-terminus of villin

3.4.6 Regulation of villin-induced cell migration by carboxyl-terminal phosphorylation site mutants

3.4.7 Tyrosine phosphorylation of carboxyl-terminal domain of villin is required for its association with PLC- $\gamma_{1}$

4.1 Tyrosine phosphorylation of villin and actin kinetics 115 
4.3 Tyrosine phosphorylation of villin: Leading the way in epithelial cell migration

List of References

Appendix A Supplemental material for chapter 2

Appendix B Supplemental material for chapter 3

Vita 


\section{List of Figures}

1.1 G-actin polymerizes to form F-actin 4

1.2 Schematic representation of villin 8

1.3 Simplified model of cell motility 14

1.4 Role of Rho GTPases in cell migration 17

$\begin{array}{ll}1.5 & \text { Dendritic nucleation model } \\ \end{array}$

2.1 HeLa Tet-Off cells expressing wild-type villin display significantly enhanced migration 36

2.2 Villin-induced cell migration is enhanced by HGF and EGF 40

2.3 Villin-induced cell migration is regulated by c-src kinase 42

2.4 Tyrosine phosphorylation of villin is required for villin-induced increase in cell migration $\quad 46$

2.5 Phosphorylation of villin at $\mathrm{Y}-60, \mathrm{Y}-81$, and $\mathrm{Y}-256$ is required for villin-induced cell migration

3.1 Tyrosine phosphorylation of wild-type and truncation mutants of villin

3.2 Identification of one major and two minor tyrosine phosphorylation sites in villin truncation mutant CT2

3.3 Tyrosine 286 and 324 are the major phosphorylation sites in the villin truncation mutant CT3

3.4 Identification of tyrosine residue 461 as the major phosphorylated residue in villin truncation mutant CT4

3.5 Tyrosine phosphorylation of wild-type and mutant villin proteins

90

3.6 Regulation of actin dynamics by the carboxyl-terminal

tyrosine phosphorylation sites in villin 
3.7 Phosphorylation of villin at Y286, Y324, Y461, Y555, Y604 or Y725 is not required for villin-induced changes in actin reorganization or cell shape change

3.8 Collectively the carboxyl-terminal tyrosine residues regulate villin-induced cell migration

3.9 Collectively the carboxyl-terminal tyrosine phosphorylation sites are required for redistribution of villin to the detergent-soluble fraction and for its association with PLC- $\gamma_{1}$

3.10 Schematic representation of the role of the carboxyl-terminal tyrosine phosphorylation sites in villin-induced cell migration

4.1 Stimulated protrusion model

A.1 There is no change in cell proliferation in HeLa cells expressing wild-type and mutant villin proteins

A.2 There is no change in villin-induced cell migration in HeLa Tet-Off cells infected with recombinant adenovirus vector

A.3 Tyrosine phosphorylation of villin is required for villin-induced increase in cell migration

A.4 Overexpression of villin does not change the F-actin content of the cells

A.5 Phosphorylation of villin at $\mathrm{Y}-60, \mathrm{Y}-81$ and $\mathrm{Y}-256$ is required for villin-induced cell migration

B.1 Individually, the carboxyl-terminal tyrosine phosphorylation sites mutants are not associated with PLC- $\gamma_{1}$

B.2 S1-S3 fragment of villin is pro-apoptotic 
Chapter 1

\section{Introduction}




\subsection{The actin cytoskeleton}

The actin cytoskeleton is a fundamental component of all eukaryotic cells. It provides force and stability and plays an integral role in a diverse array of cellular processes. Actin is mainly located in the cytoplasm, but it is also present in the nucleus where it may or may not have motor-associated functions. Actin filaments are particularly abundant beneath the plasma membrane, where they form a network that provides mechanical support, determines cell shape, and allows movement of the cell surface, thereby enabling cells to migrate. Actin exists as a globular monomer called G-actin and as a filamentous polymer called F-actin, which is a linear chain of G-actin subunits. G-actin has a molecular mass of approximately $43 \mathrm{kDa}$. Although G-actin appears globular in the electron microscope, $\mathrm{x}$-ray crystallographic analysis reveals that it is separated into two lobes by a deep cleft. The deep cleft has a tightly bound nucleotide in the center of G-actin. The nucleotide binds as a complex with either $\mathrm{Mg}^{2+}$ or $\mathrm{Ca}^{2+}$. This nucleotide is usually ATP or ADP-Pi rather than ADP. ADP-Pi binds with $\sim 10$-fold lower affinity to $\mathrm{Mg}^{2+}$-G-actin and $\sim 200$-fold lower affinity with $\mathrm{Ca}^{2+}$-G-actin compared to ATP (ATP- $\mathrm{Mg}^{2+}$ dissociation constant $(\mathrm{kd})$ is 1.2 $\mathrm{nM}$ and ATP-Ca ${ }^{2+}$ kd is $0.12 \mathrm{nM}$ ) (De La Cruz and Pollard, 1995; StrzeleckaGolaszewska, 2001). Hence, $\mathrm{Mg}^{2+}$, the dominant cation in vivo, determines the binding affinity of the nucleotide to G-actin. The nucleotide binding to G-actin affects the conformation of the molecule. In fact, without a bound nucleotide, Gactin denatures very quickly (De La Cruz and Pollard, 1995). 
G-actin has high affinity binding sites that mediate head-to-tail interactions with two other G-actin molecules, thus actin monomers polymerize to form Factin nuclei (figure 1.1). G-actin monomers are then added to the nuclei to assemble filamentous actin (F-actin). Each monomer is rotated by $166^{\circ}$ in the filaments, which gives it the appearance of a double-stranded helix (Holmes et al., 1990). Because all the actin monomers are oriented in the same direction, actin filaments have a distinct polarity, and their ends (called the barbed ends or plus ends and pointed ends or minus ends) are distinguishable from one another (figure 1.1). The addition of ions $-\mathrm{Mg}^{2+}, \mathrm{K}^{+}$, or $\mathrm{Na}^{+}-$to a solution of $\mathrm{G}$-actin will induce the polymerization of G-actin into F-actin filaments. The process is reversible: F-actin depolymerizes into G-actin when the ionic strength of the solution is lowered. The F-actin filaments that form in vitro are indistinguishable from microfilaments isolated from cells. This indicates that other factors such as actin binding proteins are not required for polymerization per se. However the presence of actin-binding proteins in cell results in a 100 times faster turnover of actin filaments. Actin polymerization from monomers proceeds in at least four reversible steps: activation, nucleation, elongation and annealing. Activation is a conformational change in G-actin monomer induced by salts. Nucleation is the association of 2 to 3 monomers to form oligomers from which polymer can grow (figure 1.1). These two steps are much slower than elongation and account for the initial lag phase in the time course of actin polymerization in vitro. Elongation involves association and dissociation of monomers from the filament. These processes can occur at either end of the filament, but association predominantly 


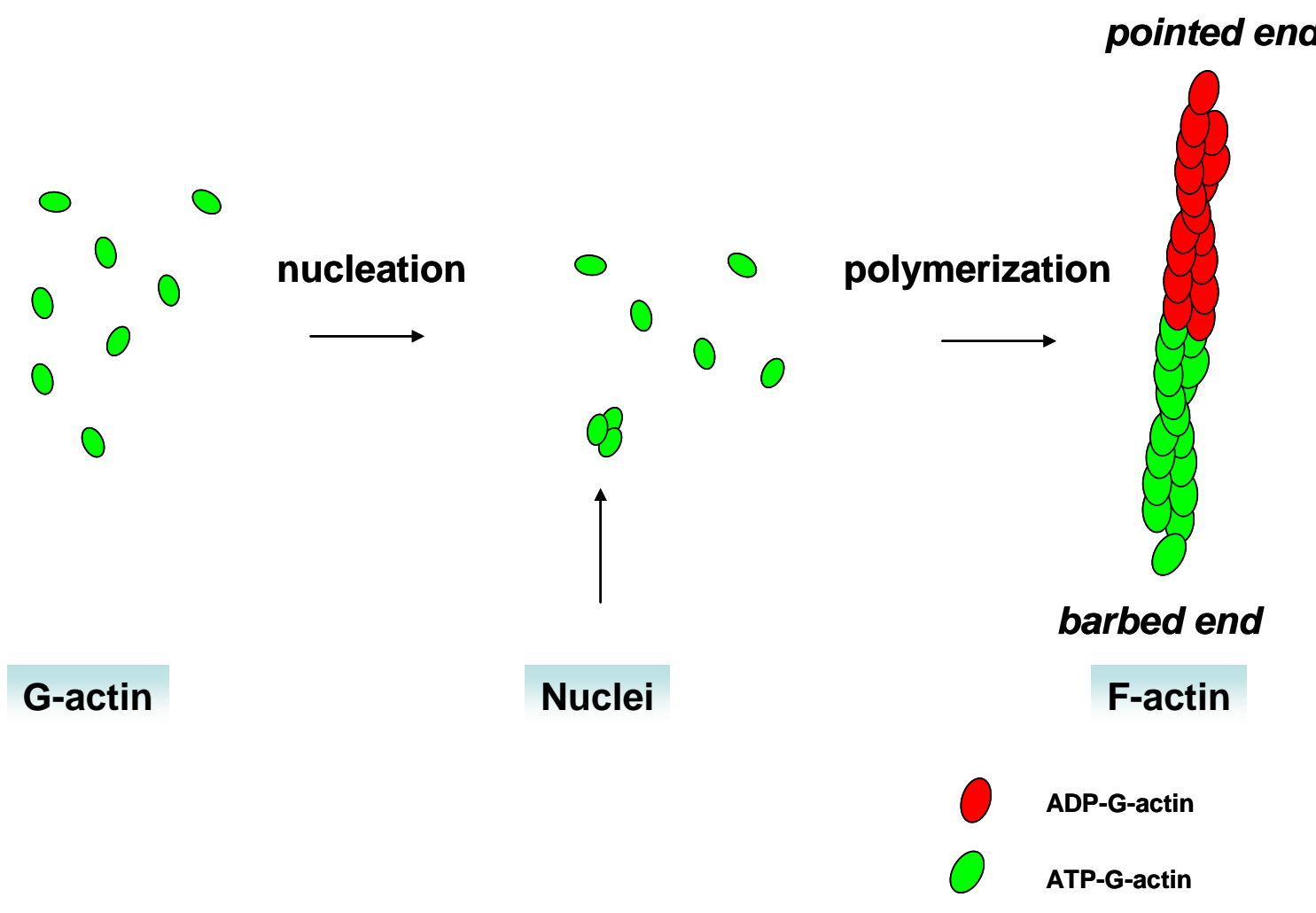

Figure $1.1 \mathrm{G}$-actin polymerizes to form F-actin. Two to three monomers of ATP bound G-actin polymerize to form the nuclei. Addition of ATP-G-actin at the barbed end in a polymerization reaction forms F-actin. Filaments age by hydrolysis of ATP bound to each ATP-G-actin subunit followed by dissociation of the $\gamma$ phosphate, which result in actin filaments with ADP-G-actin. 
occurs at the barbed end and dissociation at the pointed end as discussed below. Short actin filaments can also anneal end to end, leading over time to an increase in filament length (annealing). The nucleotide binding site of monomeric actin is almost exclusively associated with ATP in vivo. While the G-actin bound ATP is readily exchangeable, the ADP of F-actin is essentially nonexchangable (Hegyi et al., 1988). Hydrolysis of bound ATP was originally thought to be tightly coupled to the polymerization process (Wegner, 1976); however subsequent investigation (Carlier et al., 1984; De La Cruz et al., 2000) revealed that a time lag exists between the incorporation of ATP-G-actin onto the filament end and hydrolysis of the bound nucleotide. This results in the formation of an ATP-Gactin cap at the barbed end which facilitates faster polymerization at the barbed end (figure 1.1). Because actin polymerization is reversible, filaments can depolymerize by the dissociation of ADP-G-actin subunits from the pointed end. Thus, an apparent equilibrium exists between actin monomers and filaments, which are dependent on the concentration of free monomers. The rate at which actin monomers are incorporated into filaments is proportional to their concentration, so there is a critical concentration of actin monomers at which the rate of their polymerization into filaments equals the rate of dissociation. At this critical concentration, monomers and filaments are in apparent equilibrium.

As noted earlier, the two ends of an actin filament grow at different rates, with monomers being added to the fast-growing end (barbed or plus) five to ten times faster than to the slow-growing (pointed or minus) end. Because ATP-actin (barbed end) dissociates less readily than ADP-actin (pointed end), 
depolymerization of actin filaments always occurs at the pointed end. This results in a difference in the critical concentration of monomers needed for polymerization at the two ends. The critical concentration for the pointed end is 12 to 15 -fold higher than for the barbed end under physiological conditions (Wegner and Isenberg, 1983). This difference may result in the unidirectional growth of the actin filament due to a continual flux of actin subunits from the pointed to the barbed end of the filament. This reaction is called treadmilling (Wegner, 1976). Treadmilling requires ATP, with ATP-G-actin being added at the barbed end of filaments while ADP-G-actin being removed from the pointed end. The role of treadmilling at the leading edge of a moving cell was clearly demonstrated by use of fluorescent speckle microscopy (Ponti et al., 2004). Actin treadmilling at the leading edge results in the dynamic assembly and disassembly of actin filaments in an efficient unidirectional manner required for cells to change shape and move.

\subsection{Villin: An actin binding protein}

In cells the assembly and disassembly of actin filaments, and also their organization into functional higher-order networks, is regulated by a plethora of actin-binding proteins (ABPs). The turnover of actin filaments is about 100 times faster within the cell than it is in vitro, and this rapid turnover of actin plays a critical role in a variety of cell movements. Villin is an epithelial cell specific actinbinding protein. Villin is a $92.5 \mathrm{kDa}$ protein consisting of two tandem homologous halves (segments 1-3 and 4-6, S1-S6) and a head piece domain 
(segment $7, \mathrm{S7}$ ) (figure 1.2). The segments 1 through 6 are all structural repeats that are conserved amongst villin family of proteins which includes (but not limited to) gelsolin, adseverin (also known as scinderin), CapG, severin (aka fragmin), advillin, and supervillin. All of these proteins contain at least one of the structural repeats. For example gelsolin and adseverin are a six-domain ABP; CapG only has the first three domains, whereas advillin and supervillin have six domains and also share a villin-like carboxyl-terminal headpiece domain. Among all actin-binding proteins, villin is unique in presenting capping, bundling, polymerizing and severing properties in a single protein. An important question is how does villin regulate these different actin modifying activities? The work done in our lab has significantly contributed to our understanding of this important and complex question. Our studies show that the ligand-binding properties and posttranslational modification of villin protein regulate the actin modifying activities of villin (see discussion below).

\subsection{1 $\mathrm{PIP}_{2}$ regulates actin-severing, -capping and -crosslinking activity of}

villin. In vitro interaction of full length villin protein with phosphoinositide 4, 5 bisphosphate $\left(\mathrm{PIP}_{2}\right)$ was first characterized by our lab (Panebra et al., 2001). Using site directed mutagenesis we identified three $\mathrm{PIP}_{2}$ binding sites in human villin, PB1 (a.a. 112-119), PB2 (a.a. 138-146) and PB5 (a.a. 816-824), (figure 1.2). These sites contain the consensus sequence for phosphoinositide binding also found in other cytoskeletal proteins, and consists of basic amino acids with the following motif, $(X) 4(R / K)(X)(R / K R / K)$ (Kumar et al., 2004b). Two of the $\mathrm{PIP}_{2}$ 


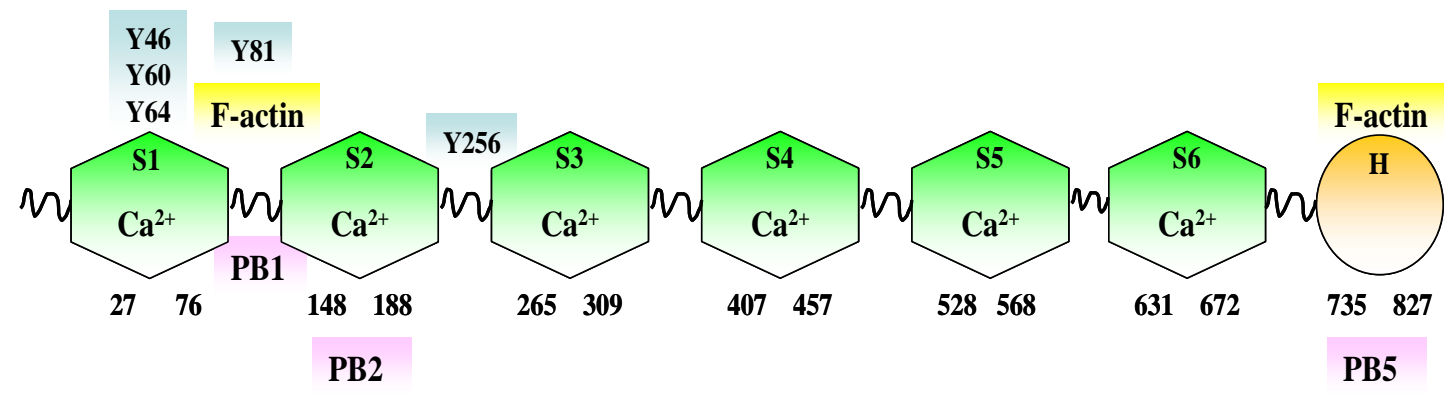

Figure 1.2 Schematic representation of villin. Segmental repeats are represented by $\mathrm{S} 1$ through $\mathrm{S} 6$. The letter $\mathrm{H}$ represents the headpiece. $\mathrm{Ca}^{2+}$ binding sites depicted in segments $\mathrm{S} 1$ represent $\mathrm{Ca}^{2+}$ binding site 1 and the site depicted in segment S2 represents the intradomain $\mathrm{Ca}^{2+}$ binding site 2 . Sites depicted in segment $\mathrm{S} 3$ through $\mathrm{S} 6$ represent the other intradomain $\mathrm{Ca}^{2+}$ binding sites in villin. F-actin binding sites, $\mathrm{PIP}_{2}$ binding sites (PB1, PB2 and PB5) and tyrosine phosphorylation sites $(\mathrm{Y}-46,-60,-64,-81$ and -256$)$ are also schematically represented. 
binding sites identified by us described actin-binding sites in villin (Kumar et al., 2004b). PB2 has been described as the site of F-actin binding to villin prior to severing, and PB5 has been identified as the F-actin-binding site involved in the bundling function of villin. However a more recent high resolution crystal structures of chicken villin head piece, has established that most of the residues in PB5 are dispensable for F-actin binding (Meng et al., 2005). Thus, PB5 is a $\mathrm{PIP}_{2}$ binding site in proximity of the F-actin binding site in villin headpiece. The third lipid-binding site PB1, lies in the vicinity of an actin monomer-binding site (a.a. 72-99) in other proteins of the villin family such as gelsolin and by homology in villin (Bretscher and Weber, 1980). PB1 and PB2 lie in the actin capping and severing domain of villin and are conserved among the actin-capping and -severing proteins, whereas PB5 lies in close vicinity of the actin cross-linking domain in the headpiece of villin and is conserved among proteins that contain a villin-like headpiece (figure 1.2). We further demonstrated that $\operatorname{Arg} 138$ in $\mathrm{PIP}_{2}$ binding domain PB2 determines $80 \%$ of the $\mathrm{PIP}_{2}$ binding and as previously reported $83 \%$ of the actin-severing activity of villin (De Arruda et al., 1992; Kumar et al., 2004b). This suggests that $\mathrm{PIP}_{2}$ binding to villin in the actin severing domain (PB2) can directly inhibit the actin-severing activity of villin by competitive inhibition. Interestingly deletion of PB1 also inhibits actin-severing activity of villin. PB1 has been described as the site that stabilizes the binding of F-actin to PB2, and $\mathrm{PIP}_{2}$ binding to PB1 results in a change in the secondary structure of PB1. This suggests that $\mathrm{PIP}_{2}$-induced structural changes in villin could also influence the actin-severing activity of villin in vivo. Like severing $\mathrm{PIP}_{2}$ also inhibited the 
capping activity of villin (Kumar et al., 2004b). $\mathrm{PIP}_{2}$ enhances the actincrosslinking activity of villin (Kumar et al., 2004b). We have recently demonstrated that $\mathrm{PIP}_{2}$ facilitates dimerization of villin (George et al., 2006). We further demonstrated that the F-actin binding site in the villin headpiece is sufficient for actin-bundling activity of villin, provided villin is dimerized. Hence, $\mathrm{PIP}_{2}$ can regulate the actin-bundling activity of villin by regulating villin selfassociation. The only actin modifying function of villin that is not regulated by $\mathrm{PIP}_{2}$ is actin-nucleation (Kumar et al., 2004b).

\subsubsection{Calcium regulates villin's actin-severing and -capping activity. It has} been shown previously that villin directly binds to calcium and can regulate the actin-capping and -cutting activities of villin (Glenney et al., 1980; Hesterberg and Weber, 1983a; Walsh et al., 1984). It has also been reported before that in the presence of calcium, there is an increase in the Stoke's radius of villin, indicative of a $\mathrm{Ca}^{2+}$-induced conformational change, which was confirmed by circulardichroism studies (Hesterberg and Weber, 1983b). The work done in our lab has demonstrated that two low affinity $\mathrm{Ca}^{2+}$-sensitive sites (aspartic acid 467 and 715 ) interact with a cluster of basic residues in the S2 domain of villin, resulting in an auto-inhibited conformation of villin. $\mathrm{A} \mathrm{Ca}^{2+}$ concentration of $>100 \mu \mathrm{M}$ causes a conformational change in villin. A similar change in villin conformation can be obtained by mutation of aspartic acid 467 and 715 to leucine, allowing villin to sever F-actin at physiological $\mathrm{Ca}^{2+}$ concentration (Kumar and Khurana, 2004). Hence, $\mathrm{Ca}^{2+}$ can regulate the structure and function of villin. With the help of site directed mutagenesis, using the NMR structure of villin 14T (Markus et al., 1994) 
and the gelsolin-actin/ $\mathrm{Ca}^{2+}$ crystal structure (Burtnick et al., 1997) our lab identified six additional sites that result in $\mathrm{Ca}^{2+}$ induced conformational changes in villin. Furthermore, we determined that villin expresses at least two types of $\mathrm{Ca}^{2+}$ sensitive sites that determine separate functional properties; site 1 (Glu-25, Asp-44, and Glu-74) regulates actin-capping, whereas sites 1 and 2 (Asp-86, Ala-93, and Asp-61), together with the intra-domain calcium-sensitive sites in villin, regulate actin-severing by villin ((Kumar et al., 2004a), (figure 1.2)). Hence, $\mathrm{Ca}^{2+}$ binding to villin can functionally dissect the actin-capping and -severing activities of villin. Although half-maximal capping is obtained at $\mathrm{Ca}^{2+}$ concentration of $1 \mu \mathrm{M}$, half-maximal severing by villin requires more than $100 \mu \mathrm{M}$ $\mathrm{Ca}^{2+}$. Our data then suggests that site 1 may be a high affinity $\mathrm{Ca}^{2+}$ binding site which could regulate the actin-capping activity of villin at low $\mathrm{Ca}^{2+}$ concentrations $(2.5 \mu \mathrm{M})$. Whereas site 2 and other intra-domain calcium sensitive sites in villin may be low affinity $\mathrm{Ca}^{2+}$ binding sites, which could regulate actin-severing activity of villin at calcium concentrations greater than $100 \mu \mathrm{M}$ (Kumar et al., 2004a). Hence, the high affinity and low affinity $\mathrm{Ca}^{2+}$ binding sites could result in a conformational change in villin from an auto-inhibited state to an active state, capable of capping $\left(2.5 \mu \mathrm{M} \mathrm{Ca}^{2+}\right)$ and severing actin filaments $\left(>100 \mu \mathrm{M} \mathrm{Ca}^{2+}\right)$ respectively.

\subsubsection{Tyrosine phosphorylation of villin regulates actin-severing,} -nucleation and -bundling activities of villin. The $\mathrm{Ca}^{2+}$ concentrations required for actin-severing by villin are in the micromolar range $(\sim 200 \mu \mathrm{M})$, which suggests that in vivo functional regulation of villin may not be regulated by $\mathrm{Ca}^{2+}$, since 
intracellular $\mathrm{Ca}^{2+}$ levels may never reach $200 \mu \mathrm{M}$ (Villereal and Palfrey, 1989). To resolve this paradox we looked at the role of post-translational modification of villin, in the form of tyrosine $(\mathrm{Y})$ phosphorylation. Our lab was the first to show that villin is tyrosine phosphorylated (Khurana et al., 1997). Five phosphorylation sites were identified by direct mutation of candidate tyrosine's $(Y)$ to phenylalanine $(F)$, namely, $Y 46,-60,-64,-81$, and -256 (figure 1.2). These sites could be phosphorylated by c-src kinase in an in vitro kinase assay, suggesting that c-src may be involved in phosphorylation of villin in cells (Zhai et al., 2002). We determined that tyrosine phosphorylated villin had enhanced actin-severing activity compared to unphosphorylated villin. Tyrosine phosphorylation of villin also resulted in actin-severing at nanomolar $\mathrm{Ca}^{2+}$ concentrations, suggesting that tyrosine phosphorylation and not $\mathrm{Ca}^{2+}$ may be the physiological regulator of villin's actin-severing activity (Zhai et al., 2002; Kumar and Khurana, 2004). Characterization of individual tyrosine phosphorylation sites in the aminoterminus of villin revealed that phosphorylation at $Y 46$ or -60 increased the actinsevering activity of villin (Zhai et al., 2002). Tyrosine phosphorylation of villin also inhibited its actin-nucleating activity (Zhai et al., 2001; Zhai et al., 2002). Since phosphorylation of villin decreases its affinity for F-actin, it provides a mechanism for inhibition of villin's actin-nucleating activity (Zhai et al., 2001; Zhai et al., 2002). Unlike severing no individual tyrosine phoshorylation site of villin regulated actin-nucleation by villin, suggesting that two or more of the identified tyrosine phosphorylation sites may regulate actin-nucleation by villin (Zhai et al., 2001; Zhai et al., 2002). Actin-crosslinking activity of villin is also inhibited by 
tyrosine phosphorylation. We have recently demonstrated that tyrosine phosphorylation of villin prevents villin dimerization, which in turn results in inhibition of actin-crosslinking by villin (George et al., 2006).

\subsection{Cell migration}

Cell migration is a key aspect of many normal and abnormal biological processes, including embryonic development, defense against infections, wound healing, and tumor cell metastasis (Trinkaus, 1988; Martin, 1997; Nodder and Martin, 1997). As shown in figure 1.3 almost universally, crawling motility involves a cycle of four steps: protrusion of the leading edge, adhesion to the substratum, retraction of the rear, and de-adhesion (Abercrombie et al., 1972). In response to various migratory cues, directional movement is initiated by polarization of the cell, as defined by the spatial segregation of molecular machineries that control the four stages of the migratory cycle. At the front of the cell, actin polymerization drives membrane protrusion to form the leading edge. Subsequently, the leading edge is stabilized by attachment to the extracellular matrix (ECM) through integrin-mediated adhesion complexes, which not only link the ECM to the actin cytoskeleton but also function as signal transduction centers that modulate cell migration. Once coupled to adhesion complexes, the actin cytoskeleton can generate the forces necessary to translocate the cell body forward. Finally, adhesive contacts at the rear of the cell must be disassembled to allow detachment of the rear to complete the migratory cycle. 

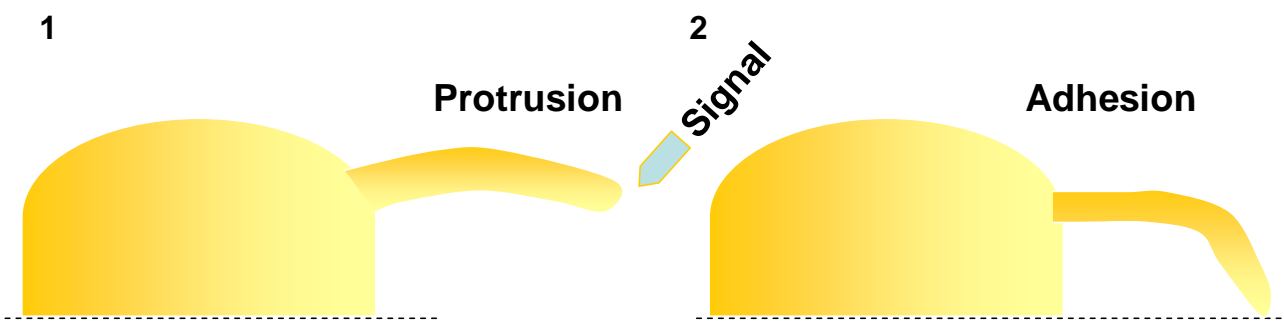

3

4

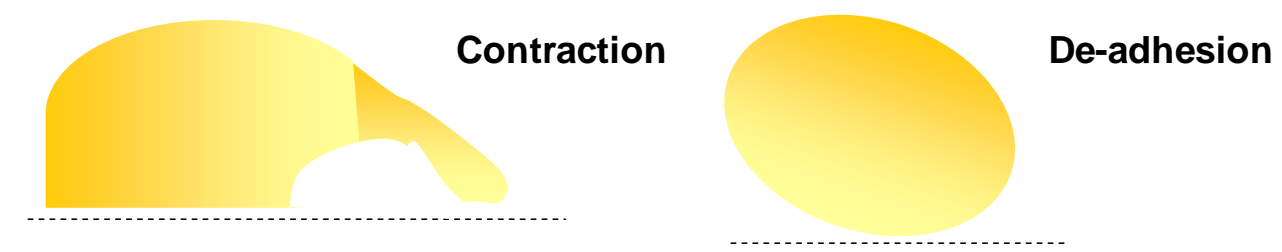

Figure 1.3 Simplified model of cell motility. Almost universally, crawling motility involves a cycle of four steps: (1) protrusion of the leading edge in response to a signal, (2) adhesion to the substratum, (3) retraction of the rear, and (4) deadhesion. 
The first step in locomotion, protrusion of a leading edge, seems to rely only on forces generated by actin polymerization pushing the plasma membrane outward. Motile cells primarily generate two types of protrusive structures, filopodia (also known as microspikes) and lamellipodia. These protrusive structures are filled with a dense core of filamentous actin, which excludes membrane-enclosed organelles. Filopodia and lamellipodia differ primarily in the way in which the actin is organized. Filopodia are essentially made up of long, bundled actin filaments, which are reminiscent of those in microvilli but longer and thinner, as well as more dynamic. Lamellipodia are sheet-like structures, they contain an orthogonally cross-linked mesh of actin filaments, most of which lie in a plane parallel to the solid substratum. (DeMali and Burridge, 2003). Filopodia are postulated to increase directional motility by acting as environmental sensors (Bryant, 1999). Lamellipodia contains all of the machinery that is required for cell motility. Understanding how actin is assembled at the leading edge can provide important insight into the molecular mechanism(s) of cell migration. The next section describes the machinery that is required for the initiation and formation of lamellipodia.

\subsubsection{Role of Rho family GTPases in lamellipodia formation. The signalling} pathways that initiate lamellipodia formation are somewhat obscure; however it is clear that Rho family guanosine tri-phosphatases (GTPases) play a central role. These proteins (Rac, Cdc 42 and Rho) act as molecular switches cycling between an active GTP and inactive GDP-bound forms. Despite being membrane-anchored via their isoprenylated carboxy-termini, Rho GTPases 
rapidly translocate between membrane and cytosolic compartments. The role of Rho family GTPases has been revealed by simple microinjection experiments. Microinjection of activated Rac into fibroblasts induced dramatic lamellar ruffling driven by de novo actin polymerization (Ridley and Hall, 1992; Machesky and Hall, 1997). Dominant negative Rac mutant blocked motility of macrophages and prevented membrane ruffling in stimulated fibroblasts (Ridley and Hall, 1992). Injection of Cdc 42 into fibroblast resulted in the formation of filopodia (Kim et al., 2000). When Rho protein was microinjected into fibroblasts it resulted in the rapid assembly of stress fibers and focal adhesions but did not have any influence on the lamellipodia formation. Hence, in response to various migratory cues Rac and Cdc 42 are activated at the leading edge of a motile cell and are crucial for lamellipodia protrusion. In contrast Rho is activated at the trailing edge of the cell and is responsible for cell contraction at the trailing edge (figure 1.4).

1.3.2 Role of actin in lamellipodia formation. Rapid changes in the levels of Factin and G-actin that occur in a spatially and temporally controlled fashion are involved in the morphological changes in living cells. Observation of actin dynamics in motile cells indicates that not only are actin filaments actively polymerizing beneath the plasma membrane at the leading edge, but also the turnover of actin filaments is faster (for instance during actin treadmilling) in the lamellipodia than in other regions of the cell (Condeelis, 1993). Lamellipodia extension requires assembly of a specialized network of actin filaments at the leading edge of the cell. F-actin in lamella is mainly present in the form of crosslinked network which are several microns long and organized into two nearly 


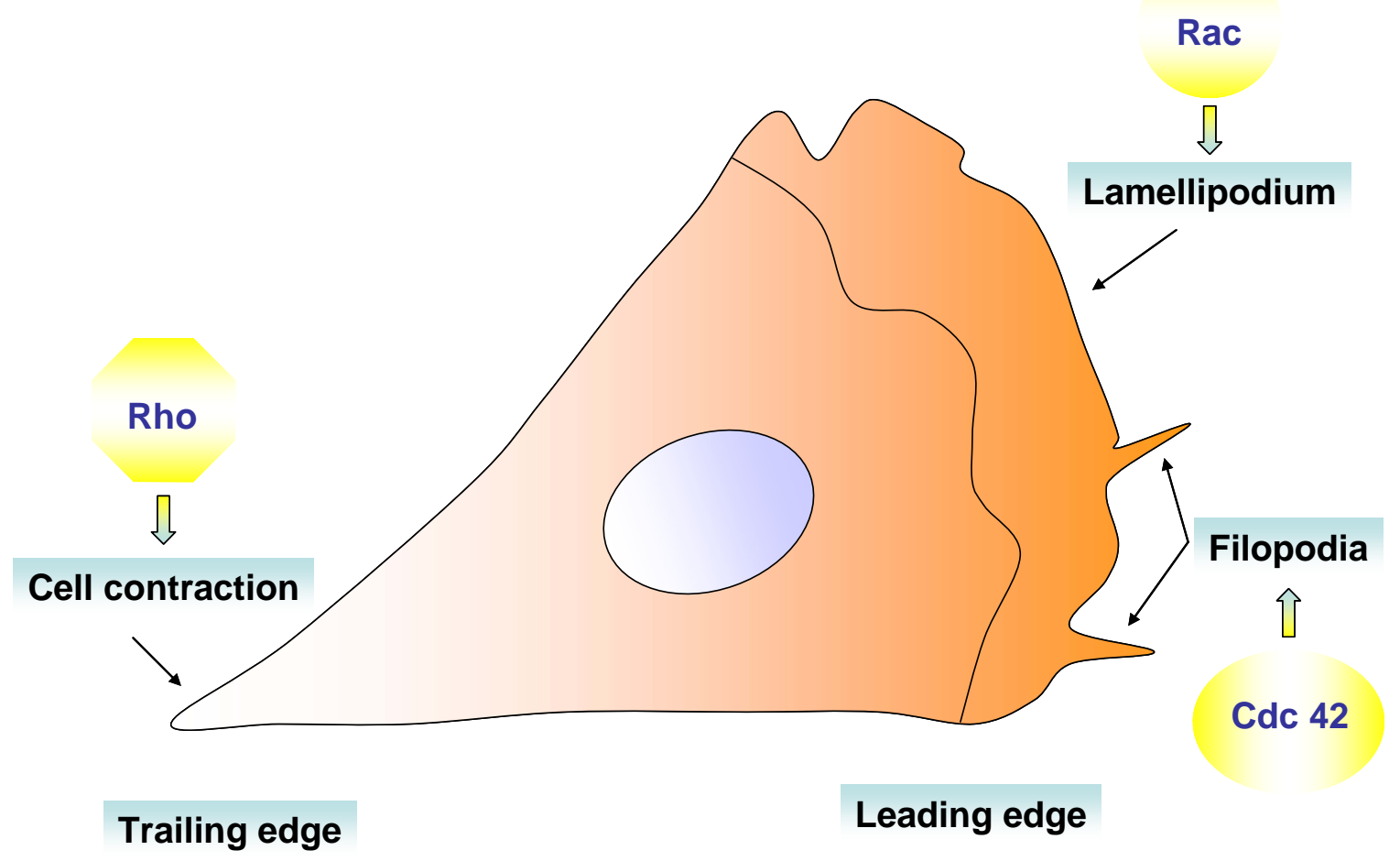

Figure 1.4 Role of Rho GTPases in cell migration. Rac and Cdc 42 are activated at the leading edge of a motile cell. Activation of Rac results in the formation of lamellipodia, whereas activation of Cdc 42 results in the formation of filopodia. Rho is activated at the trailing edge of the cell and is responsible for cell contraction at the trailing edge. 
parallel two-dimensional arrays, approximately orthogonal to each other (Small et al., 1995). Closer to the membrane (within $1 \mu \mathrm{M}$ ) is a population of very short filaments that are organized into an extensive network of Y-branches (Svitkina and Borisy, 1999). This specialized arrangement of F-actin in the lamella is responsible for the formation and growth of lamellipodia in a moving cell.

1.3.3 Role of actin binding proteins in lamellipodia formation. Cells are endowed with a rich variety of actin binding proteins, falling into more than 60 classes. Actin and a limited subset of actin binding proteins can reconstitute motility in a purified system. These include ADF/cofilin, Cap Z, Arp2/3 complex, an activator of Arp2/3 complex and profilin (Loisel et al., 1999). Many other actin binding proteins including villin family of proteins have been shown to play an important role in cell migration and lamellipodia formation, but for purposes of simplicity, I will concentrate on these core proteins.

Profilin binds ATP-actin monomers in a stable 1:1 complex and is essentially a high-affinity monomer binding protein. Rather than sequestering actin monomers (which is also achieved by other monomer binding proteins like thymosin), the main function of profilin is to promote assembly of actin filaments in cells (Lu and Pollard, 2001). Profilin in complex with ATP-G-actin is postulated to assist in addition of monomers exclusively at the barbed end of an actin filament. After the complex binds transiently to the filament, the profilin dissociates from actin. Profilin also binds to proline-rich sequences that are commonly found in membrane-associated signaling proteins such as formins, Vasp and Mena. This interaction, which does not inhibit profilin binding to G- 
actin, localizes profilin-actin complexes to the membrane (Lu and Pollard, 2001). Profilin also promotes assembly of actin filaments by acting as a nucleotideexchange factor. Profilin is the only actin binding protein that allows the exchange of ADP for ATP. Profilin regulates actin treadmilling as it converts the ADP-G-actin released from the pointed end of actin filaments to ATP-G-actin that can be added to the barbed end of actin filaments. (Pollard and Borisy, 2003). Hence, profilin plays an important role in actin polymerization at the leading edge.

At the leading edge the barbed ends of actin filament are always oriented towards the membrane. As a result, actin polymerization at the barbed end generates membrane protrusion. Most filament depolymerization occurs at sites located well behind the leading edge. ADF/Cofilin accelerates the actin depolymerization at the pointed end thus playing an important role in cell migration. Cofilin binds cooperatively and preferentially to pointed end of actin filaments, containing ADP-actin, and not at the barbed end containing ATP-actin, causing enhanced rate of monomer dissociation from the pointed end. The ADPactin released from the pointed end is then converted to ATP-actin by profilin as described above. Hence, ADF/cofilin along with profilin plays an essential role in actin treadmilling, which maintains efficient, unidirectional actin-polymerization in the lamellipodium.

Cap $Z$ a heterodimeric capping protein is a ubiquitous barbed end capping protein which prevents actin assembly from barbed ends. Capping may appear counter-productive in cell motility, since it antagonizes elongation of barbed ends, 
the driving force for motility. However, capping makes two important contributions to actin-driven motility. First, capping proteins limit the length of the growing branches. Short filaments are stiffer than long filaments and therefore more effective at pushing the membrane. Second, capping controls where actin filaments "push". Since only those barbed ends in contact with the lamellipodial surface are effective in generating propulsive force, global capping of barbed ends avoids non-productive consumption of actin subunits elsewhere in the cell and funnels subunits from diverse points of disassembly to a limited number of growing barbed ends (Carlier and Pantaloni, 1997; Cooper and Schafer, 2000; Pollard and Borisy, 2003).

The formation and growth of lamellipodia in a moving cell needs to be fuelled by continuous new F-actin filaments nuclei. Severing of existing filaments, uncapping of existing filaments or de novo nucleation can all result in the formation of new actin nuclei. However de novo creation of new nuclei is now considered to be the dominant mechanism in the leading edge (Zigmond, 1996). Arp2/3 complex has the ability to form de novo actin filaments. Arp $2 / 3$ complex is a stable assembly of two actin-related proteins, Arp2 and Arp3, with five novel subunits called ARPC1, ARPC2, ARPC3, ARPC4 and ARPC5 (Machesky et al., 1994). Biochemical and cell biology experiments established that the Arp $2 / 3$ complex caps pointed ends and initiates growth in the barbed end direction at $70^{\circ}$ branches identical to actin filament branches (Y-branches) seen in growing lamellipodia (Mullins et al., 1998). The Arp2/3 complex sits at the $Y$ branch between the filaments and stabilizes the cross-link both in vitro and in vivo. 
Coordination of these two activities of the Arp2/3 complex is thought to simultaneously generate new filaments and connect them together in approximately orthogonal networks in cells.

Purified Arp2/3 complex possesses little activity on its own, but it can be activated by a class of proteins called nucleation promoting factors (NPFs) that are highly conserved from bacteria to mammals (Mullins et al., 1998). These include the Listeria monocytogenes ActA protein (Welch et al., 1998), and the evolutionary conserved Wiskott-Aldrich syndrome protein (WASP), among others (Rohatgi et al., 1999; Winter et al., 1999). Based on this information a model has been proposed called the "dendritic model" which also explains the quantitative mechanism of lamellipodia formation and growth (figure 1.5). The model proposes that, in the absence of new actin nuclei, cytoskeletal components are held in a metastable state, poised for assembly. Activation of WASP family proteins activates Arp2/3 complex to create new barbed ends on existing filaments, at a constant rate. These filaments grow rapidly and push the membrane forward. After a short time, growth of barbed ends is terminated by capping. Consequently the system requires continuous activation of new Arp2/3 complex, because it is consumed by incorporation into a network that grows for a very limited time. If the rate of nucleation drops to zero, capping stops polymerization automatically. Constitutive ATP hydrolysis within actin filaments and dissociation of phosphate triggers severing and depolymerization at pointed ends of actin filament by ADF/cofilin at a rate that is controlled by some of the same signals that stimulate assembly. Nucleotide exchange catalyzed by profilin 


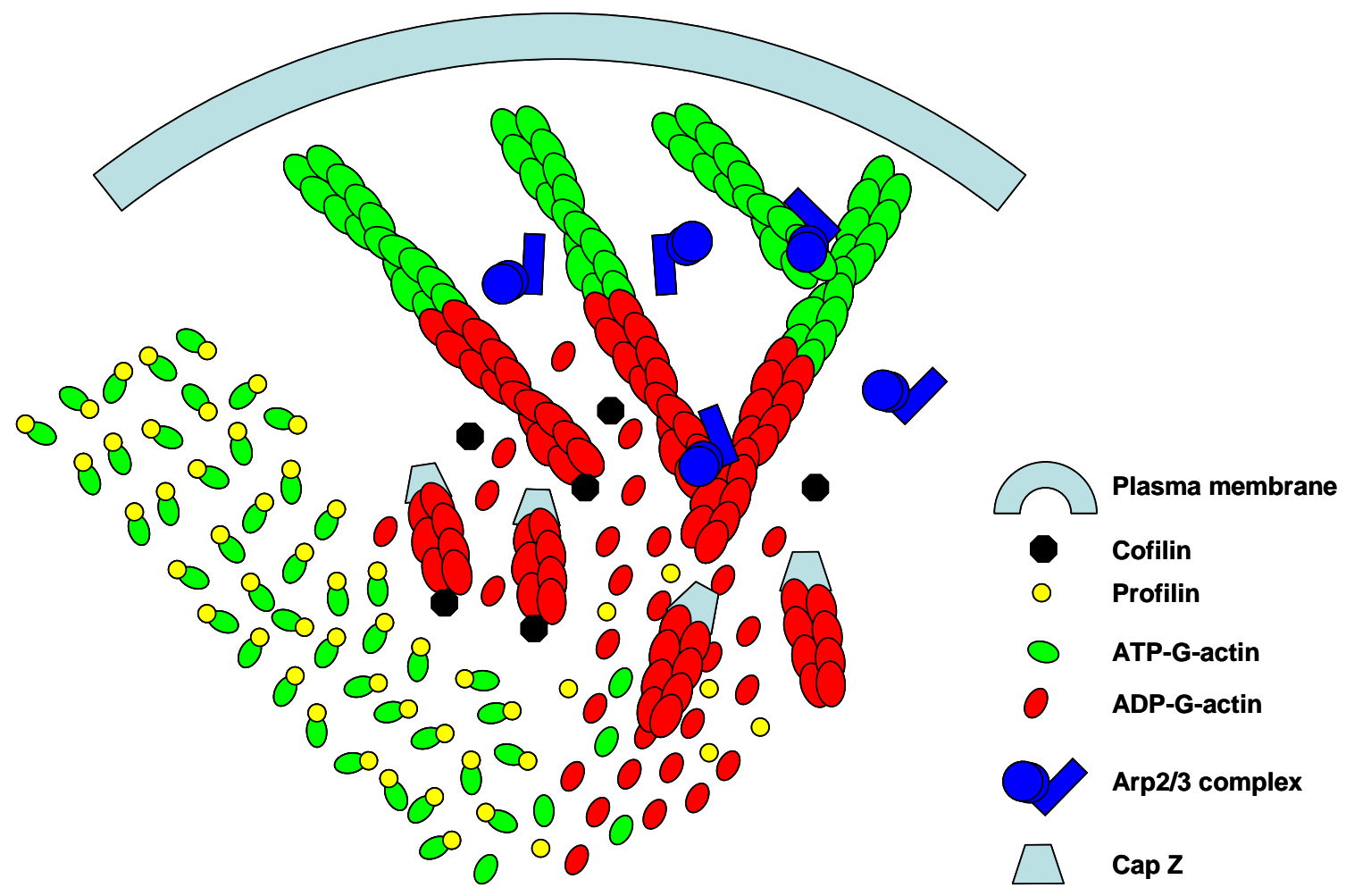

Figure 1.5 Dendritic nucleation model. Activation of Arp2/3 complex generates a new F-actin branch on a pre-existing actin-filament. Rapid growth occurs at the barbed end of actin filaments which pushes the membrane forward. Filaments age by hydrolysis of ATP bound to each ATP-G-actin subunit followed by dissociation of the $\gamma$ phosphate, which results in actin filaments with ADP-G-actin. Profilin catalyzes the exchange of ADP for ATP returning subunits to the pool of ATP-actin bound to profilin, ready to elongate barbed ends as they become available. 
recycles ADP-actin subunits back to the ATP-actin monomer pool, and is rapidly added to the fast growing barbed end of actin filament. Thus, in a continuously moving lamellipodia, assembly and disassembly are balanced (Pollard et al., 2000). If the actin depolymerization at the pointed end is slower compared to actin polymerization at the barbed end, the pool of ATP-G-Actin will diminish thus preventing actin polymerization and inhibiting cell migration. 


\section{Chapter 2}

\section{Regulation of cell motility by tyrosine phosphorylated villin ${ }^{1}$}

1 Permission to reproduce by The American Society for Cell Biology. Tomar, A., Wang, Y., Kumar, N., George, S., Ceacareanu, B., Hassid, A., Chapman, K.E., Aryal, A.M., Waters, C.M., and Khurana, S. (2004). Regulation of cell motility by tyrosine phosphorylated villin. Mol. Biol. Cell 15, 4807-4817. 


\subsection{Introduction}

Villin, an epithelial cell-specific protein belongs to a family of actin binding proteins that contain segments that display internal homology with each other (Arpin et al., 1988). The amino terminal core of villin retains the actin-capping and -severing functions of villin while the carboxyl terminal headpiece enables villin to crosslink actin filaments. The actin modifying properties of villin are regulated in vitro by calcium (Northrop et al., 1986a), phosphoinositides (Janmey and Matsudaira, 1988; Kumar et al., 2004a) and tyrosine phosphorylation (Zhai et al., 2001; Kumar and Khurana, 2004). It has been assumed for several years that villin's actin-bundling and not actin-severing functions are important because unphysiologically high $\mathrm{Ca}^{2+}$ concentrations $(200 \mu \mathrm{M})$ are required to activate villin's actin-severing activity. However, studies done with the villin knock-out mice suggest that in the absence of villin, the actin-bundling properties associated with villin can be substituted by other proteins in the microvilli (Pinson et al., 1998); on the other hand the actin-severing activity of the microvilli is lost (Ferrary et al., 1999). In recent years we have demonstrated that villin's actin modifying functions can be regulated in vitro by tyrosine phosphorylation and $\mathrm{PIP}_{2}$, suggesting that villin has the potential to function as a link between receptor activation and actin cytoskeleton reorganization even in the absence of high calcium (Zhai et al., 2001; Kumar and Khurana, 2004; Kumar et al., 2004a). In addition, we have recently demonstrated that the auto-inhibited conformation of villin can be released by tyrosine phosphorylation of villin (as opposed to high 
$\mathrm{Ca}^{2+}$ ) allowing it to sever actin at physiological $\mathrm{Ca}^{2+}$ concentrations (Kumar and Khurana, 2004). These results suggest that tyrosine phosphorylation rather than high calcium may be the mechanism by which villin severs actin in vivo. From our in vitro studies we developed the hypothesis that in vivo villin may be a key factor integrating information from external stimuli that could affect the plasticity of the actin cytoskeleton from a more rigid to a dynamic network, and that these spatial and temporal changes in the physical properties of the cytoskeleton could enhance cell motility (Panebra et al., 2001; Zhai et al., 2001; Kumar et al., 2004a; Kumar et al., 2004b). Further, that villin's ability to cycle between active (phosphorylated) and inactive (non-phosphorylated) forms may be a critical feature of its mechanism of action in cell migration.

The current study was designed to determine specifically the role of villin overexpression on cell morphology and cell migration. Overexpression of other proteins of the villin superfamily including gelsolin and CapG have been demonstrated to increase the motile phenotypes of cells (Hartwig et al., 1989; Aizawa et al., 1996; Chen et al., 1996; Furnish et al., 2001). Conversely, the gelsolin-null mice and other mutant cell lines lacking these proteins have been shown to exhibit decreased rates of cell motility (Witke et al., 1995; Lu et al., 1997; Chellaiah et al., 2000). To confirm our hypothesis that villin and its ligandbinding activities described by in vitro experiments are mechanistically important to villin's relationship to cell motility, we used the villin-null HeLa cells to overexpress villin using a tetracycline-regulated system. We demonstrate that villin participates in actin reorganization and cell migration. Further we 
determined that tyrosine phosphorylation of villin is essential to its role in cell migration. We have previously identified an area in the amino terminus of villin as the site of phosphorylation. Four major sites of phosphorylation were identified in this region by direct mutation of candidate tyrosine's $(Y)$ to phenylalanine $(F)$, namely Y-46, $-60,-81$, and -256 (Zhai et al., 2002). Mutation of any of these phosphorylation sites inhibits the ability of villin protein to polymerize actin filaments in vitro (Zhai et al., 2002). Based on our previous studies, phosphorylated villin could regulate cell motility by modifying actin assembly by distinct mechanisms, including lowering the binding affinity for F-actin, inhibiting polymerization of existing actin nuclei, and/or cutting pre-existing filaments to generate new actin nuclei (Zhai et al., 2001; Zhai et al., 2002) In this study, we investigated the role of each tyrosine phosphorylation site of villin in actin reorganization and cell migration by establishing HeLa cell lines stably expressing wild-type or each of the phosphorylation site mutants (tyrosine to phenylalanine) of villin. The ability of each mutant of villin (tyrosine to glutamic acid) to rescue actin organization and cell migration was analyzed to confirm the essential phosphorylation sites responsible for each of these biological processes. We demonstrate that villin participates in actin reorganization and cell migration by utilizing more than one phosphorylation site. We propose that villin tyrosine phosphorylation is coordinated with actin remodeling, where it may serve to integrate and transduce signals involved in cell migration. Since there is significant homology between villin and other actin-capping and -severing proteins of its family that are also tyrosine phosphorylated (De Corte et al., 1997) 
and are known regulators of cell motility (Cunningham, 1995; Sun et al., 1995; Arora and McCulloch, 1996), the results of our study provide a structural basis for the mechanism of cell motility and help to understand the role of phosphorylation in this function.

\subsection{Materials}

HeLa Tet-Off cells stably expressing the tTA tetracycline-controlled transactivator protein, G418, hygromycine, doxycycline, the eukaryotic expression vector pTRE-6xHN, and the selection vector pTK-Hyg were purchased from Clonetech. Lipofectine was purchased from Invitrogen; monoclonal antibodies to phosphotyrosine (clone PY-20) were from ICN; monoclonal antibodies to villin were from Transduction Laboratories; hepatocyte growth factor (HGF), epidermal growth factor (EGF), 4-amino-5-(4-chlorophenyl)7-(t-butyl)pyrazolo(3,4-d)pyrimidine (PP2), and 4-amino-7-phenylpyrazolo (Forney et al., 1999) pyrimidine (PP3) were purchased from Calbiochem; Alexa Fluor 568 Phalloidin, was acquired from Molecular Probes; fluorescein (FITC)conjugated affinity purified donkey anti-mouse IgG was purchased from Jackson ImmunoResearch Laboratories; QuikChange site-directed mutagenesis kit was purchased from Strategene. Src cDNA (dominant negative); (K296R/Y528F mutation) and src monoclonal antibodies were purchased from Upstate Biotechnology. Cell culture reagents were purchased from GIBCO-BRL (Grand Island, NY). All other chemicals were from Sigma or Invitrogen. 


\subsection{Methods}

2.3.1 Villin cDNA construct. Human villin cDNA containing the entire coding sequence cloned in the prokaryotic expression vector pGEX2T (Panebra et al., 2001) was amplified by PCR using the primers GAL1 5'GTTCCGCGGTCGACCATGACC3') and GAL2 (5'TCAATTGGTCTAGAACTAATA3'). These primers contain sequences for the restriction enzymes Sal I and Xba I, respectively. The Sal I and Xba I digested amplicon was ligated into the plasmid pTRE-6xHN that was digested with the same restriction enzymes. The ligation reaction was used to transform XL1-Blue competent cells and plasmids were isolated from several colonies. The plasmids were checked by sequential double digestion with Sal I and Xba I respectively. Plasmids that excised the insert were used for sequencing.

2.3.2 Phosphorylation site mutants of villin. Human villin cloned in the eukaryotic expression vector pTRE-6xHN was used as a template to mutate the known tyrosine phosphorylation sites in human villin. The identified phosphorylatable tyrosine $(\mathrm{Y})$ residues in full-length human villin were changed to phenylalanine $(F)$ or glutamic acid $(E)$ by designing complementary primers. Tyrosines at positions $46,60,81$, and 256 were replaced with phenylalanine or glutamic acid using the QuikChange site-directed mutagenesis kit to make single-base changes from TAT and TAC to TTT and TTC, or GAA and GAG respectively. The mutation primers were as follows:

Y46F, 5'- GATGGTGACTGCTTCATCATCCTGGC-3' (forward) and 
5'-GCCAGGATGATGAAGCAGTCACCATC- 3' (reverse);

Y60F, 5'-AGCAGCCTGTCCTTTGACATCCACTAC- 3' (forward) and 5'-GTAGTGGATGTCAAAGGACAGGCTGCT-3' (reverse);

Y81F, 5'-GCAGCTGCCATCTTCACCACACAGATG-3' 4 (forward) and 5'-CATCTGTGTGGTGAAGATGGCAGCTGC- 3' (reverse); Y256F, 5'-TGCACTCAAACTGTTCCATGTGTCTGAC- 3' (forward) and 5'-GTCAGACACATGGAACAGTTTGAGTGCA- 3' (reverse).

Y46E, 5'-TCGATGGTGACTGCGAAATCATCCTGGCTATC-3' (forward) and 5'-GATAGCCAGGATGATTTCGCAGTCACCATCGA (reverse);

Y60E, 5'-AGCAGCCTGTCCGAAGACATCCACTAC-3' (forward) and 5'-GTAGTGGATGTCTTCGGACAGGCTGCT- 3' (reverse);

Y81E, 5'-GCAGCTGCCATCGAAACCACACAGATG- 3' (forward) and 5'-CATCTGTGTGGTTTCGATGGCAGCTGC- 3' (reverse);

Y256E, 5'-TGCACTCAAACTGGAACATGTGTCTGAC-3' (forward) and 5'-GTCAGACACATGTTCCAGTTTGAGTGCA-3' (reverse). The introduction of the desired codon was confirmed by sequencing.

\subsubsection{Preparation of recombinant adenovirus expressing dominant negative}

c-src kinase. Replication-deficient recombinant type 5 adenovirus expressing dominant negative c-src was prepared using an adenoviral preparation kit developed by the University of lowa (Anderson et al., 2000). Briefly, plasmid cDNA (containing dominant negative c-src) was digested with Hind III and Bam $\mathrm{HI}$ and ligated into the pShuttle vector pacAd5 CMV K-N pA. Recombinant homogenous virus was generated in HEK 293 cells by homologous 
recombination. Nucleotide sequencing was used to confirm the construct. Adenoviral titers were determined by measuring their cytopathic effects in HEK 293 cells. HeLa cells were mock infected (with Ad-EGFP) or infected with the dominant negative c-src kinase at a multiplicity of infection of 100 using standard protocol (White et al., 1984). Cell lysates were analyzed by western blotting for csrc kinase expression.

\subsubsection{Transfection of Tet-Off HeLa cells with full-length and mutant villin} cDNA. HeLa Tet-Off cells described by Gossen and Bujard (Gossen and Bujard, 1992) and stably transfected with the tTA tetracycline-controlled transactivator were purchased from Clontech. The pTRE-6xHN full-length and mutant villin constructs were co-transfected with a selection plasmid carrying the hygromycine resistance gene using lipofectine. Transfected cells were selected by growing in media containing hygromycine $(400 \mu \mathrm{g} / \mathrm{ml})$ and, G418 $(100 \mu \mathrm{g} / \mathrm{ml})$. Several clones were obtained, all expressing villin in a doxycycline-dependent manner. Clones expressing comparable amounts of wild-type and mutant villin proteins were selected for further studies. For all the experiments cells transfected with wild-type or mutant-villin proteins were grown in the absence or presence of doxycycline $(1.0 \mu \mathrm{g} / \mathrm{ml})$.

2.3.5 Cell motility assay. To measure cell motility, HeLa cells transfected with villin were seeded in 6-well plates and cultured in the absence or presence of doxycycline. Cell migration was measured as described previously (Waters and Savla, 1999). Briefly, confluent monolayers were scraped with a plastic pipette tip 
across the diameter of the well to produce wounds of approximately $800 \mu \mathrm{m}$ width. Cells were rinsed to remove cellular debris, and images were obtained at the initial time of wounding and at various times up to $12 \mathrm{~h}$ post-wounding. Images of the wounds were collected with a NikonTE300 inverted microscope equipped with a CoolSnap FX CCD camera (Roper Scientific, Trenton, NJ), an Optiscan ES102 motorized stage system (Prior Scientific, Rockland, MA), and a Pentium III computer with Metamorph image analysis software (Universal Imaging Corporation, Dowington, PA). Images were collected by programming the $\mathrm{X}, \mathrm{Y}$, and $\mathrm{Z}$ coordinates of each wound location allowing the stage to return to the precise location of the original wound. Data are expressed as a percentage of the original wound width to normalize variability in wounding from well to well, although similar size initial wounds were observed from experiment to experiment. Wound width measurements were averaged from two regions of the same well and the mean treated as a single data point. Comparisons between mean values were made using one-way repeated-measures analysis of variance and Turkey's modified $t$ test (Bonferroni criteria) with $p<0.05$ considered significant.

2.3.6 Phospho-villin antibodies. Recombinant villin that was tyrosine phosphorylated (VILT/WT) or not (VIL/WT) was prepared as described before (Panebra et al., 2001). A phospho-villin antibody was raised using a commercial facility (Biosource International). One of the antibodies, clone \#VP-70782 was affinity-purified and its specificity for the phospho-epitope was examined using western analysis. Since the villin monoclonal antibody cannot be used to 
immunoprecipitate villin, the phospho-villin antibody was used to immunoprecipitate villin from HeLa cells transfected with wild-type villin protein. Additional immunoprecipitation studies were done with a commercially available phospho-tyrosine antibody (clone PY-20).

2.3.7 Immunofluorescence microscopy. HeLa Tet-Off cells expressing wildtype or mutant villin proteins were cultured on coverslips and fixed in $3.7 \%$ formaldehyde in PBS for 15 min. The slides were washed twice with phosphate buffered saline and permeabilized by incubation in PBS containing $0.2 \%$ Triton $\mathrm{X}-100$ and $0.5 \%$ normal serum for $5 \mathrm{~min}$ at $4^{\circ} \mathrm{C}$. The fixed and permeabilized cells were washed three times with PBS (10 min each) and blocked with $0.1 \%$ bovine serum albumin in TBST $(20 \mathrm{mM}$ Tris, $\mathrm{pH} 7.2,150 \mathrm{mM} \mathrm{NaCl}$ and $0.1 \%$ Tween-20) for $30 \mathrm{~min}$. Cells were incubated with villin monoclonal antibody (1:100) and Alexa Fluor 568 was included to record distribution of F-actin. The cells were then incubated with a secondary antibody, fluorscein-5-isothiocyanate (FITC)-conjugated affinity purified donkey anti-mouse IgG. The fluorescence was examined by confocal laser-scanning microscopy (Zeiss LSM 5 PASCAL).

\subsubsection{F-actin content measurements in HeLa cells expressing wild-type or}

mutant villin proteins. The F-actin content of cells was determined as described by Cunningham (Cunningham, 1995). Briefly, cells were fixed with $3.7 \%$ paraformaldehyde, permeabilized with $0.5 \%$ Triton X-100, and stained with 250 nM Alexa-Phalloidin at $37^{\circ} \mathrm{C}$ for 30 min. Ethidium bromide-binding DNA was used at a final concentration of $10 \mu \mathrm{M}$ to label the DNA at room temperature for $5 \mathrm{~min}$. 
The cells were washed with PBS, scraped in $500 \mu \mathrm{l}$ of methanol and the fluorescence recorded. The Alexa Phalloidin was quantitated at an excitation fluorescence recorded. The Alexa Phalloidin was quantitated at an excitation wavelength of $578 \mathrm{~nm}$ and emission of $600 \mathrm{~nm}$, while the DNA was measured at excitation of $525 \mathrm{~nm}$ and emission of $605 \mathrm{~nm}$. The relative F-actin content was quantified as the ratio of Alexa-phalloidin fluorescence intensity to ethidium bromide fluorescence intensity.

2.3.9 Cell proliferation measurement. Cell proliferation was measured by bromodeoxyuridine (BrdU) labeling by quantitating BrdU incorporation into newly synthesized DNA of replicating cells using the BrdU in-situ detection kit according to the instructions of the manufacturer (BD PharmigenTM). Wounded monolayers were incubated with $\mathrm{BrdU}(10 \mathrm{mM})$ for $1 \mathrm{~h}, 10 \mathrm{~h}$ post-wounding. The cells were fixed, washed, permeabilized and then incubated with $0.3 \% \mathrm{H} 2 \mathrm{O} 2$ to block endogenous peroxidase. Immuno-detection of incorporated BrdU label was done fixed, washed, permeabilized and then incubated with $0.3 \% \mathrm{H} 2 \mathrm{O} 2$ to block endogenous peroxidase. Immuno-detection of incorporated BrdU label was done using a biotinylated anti-BrdU antibody that specifically recognizes 5-bromo-2'deoxyuridine showing no cross-reactivity with thymidine or uridine. BrdU incorporation was detected by using the chromagen diaminobenzidine. Slides were counterstained with hematoxylin. 


\subsection{Results}

\subsubsection{Cells expressing villin migrate faster than villin-null cells. Cells}

expressing villin migrate faster than villin-null cells. HeLa Tet-Off cells were cotransfected with full-length human villin cloned in pTRE-6xHN and pTK-Hyg and subjected to selection with hygromycine B. Several stable transfectant lines were expanded and screened for efficient gene induction by removal of doxycycline. A representative transfectant of the villin gene was compared with parental cells for the gene expression in the absence or presence of doxycycline. western blot analysis showed that the HeLa Tet-Off cells transfected with human villin cDNA can be induced to express significant amounts of full-length villin protein when cultured without doxycycline, while cells cultured in the presence of doxycycline $(1.0 \mu \mathrm{g} / \mathrm{ml})$ do not express villin (VIL/NULL) (figure 2.1A). Untransfected HeLa cells as well as HeLa cells transfected with vector alone do not express villin. A wound-healing assay was used to determine the kinetics of migration of HeLa Tet-Off cells overexpressing villin both during basal conditions as well as chemotactic migration. Cell migration was recorded between time 0 and $24 \mathrm{~h}$ post-wounding for cells transfected with wild-type villin cultured in the presence or absence of doxycycline under low serum (1.0\% fetal bovine serum) conditions. Cell motility was judged from the reduction of the wound width $0-24 \mathrm{~h}$ after the wound was made. Migration of cells expressing villin (VIL/FL) was complete at $12 \mathrm{~h}$ post wounding (figure 2.1B). In contrast a significant delay in the ability of the VIL/NULL cells (villin transfected cells cultured in the presence 
Figure 2.1 HeLa Tet-Off cells expressing wild-type villin display significantly enhanced migration. (A) HeLa Tet-Off cells were stably transfected with fulllength human villin cDNA. Cells cultured in the absence of doxycycline express villin (VIL/FL), whereas cells cultured in the presence of doxycycline $(1.0 \mu \mathrm{g} / \mathrm{ml})$ do not express villin (VIL/NULL). Expression of villin protein was followed 0-150 h after the addition of doxycycline. western analysis was done using monoclonal villin antibodies. Data are representative of three experiments with similar results. (B) Equal numbers of wild-type (VIL/FL) and villin null (VIL/NULL) cells were cultured to confluence. Cells were denuded with a pipette tip and migration of the remaining cells into the wound is shown $0-12 \mathrm{~h}$ after the wound was introduced under low-serum condition ( $1.0 \%$ fetal bovine serum). Wound repair was measured as a percentage of initial wound area. Unlike villin-null cells, cells expressing villin seal the wound completely $12 \mathrm{~h}$ post wounding. Bar, $40 \mu \mathrm{m}$. (C) HeLa cells expressing villin migrates faster than villin-null cells at all time points. Migration distance was determined by taking two independent measurements from each well in a total of 24 wells. The error bars are the measured SEM and the asterisk denotes statistically significant values $(p<0.05, n=24)$. 

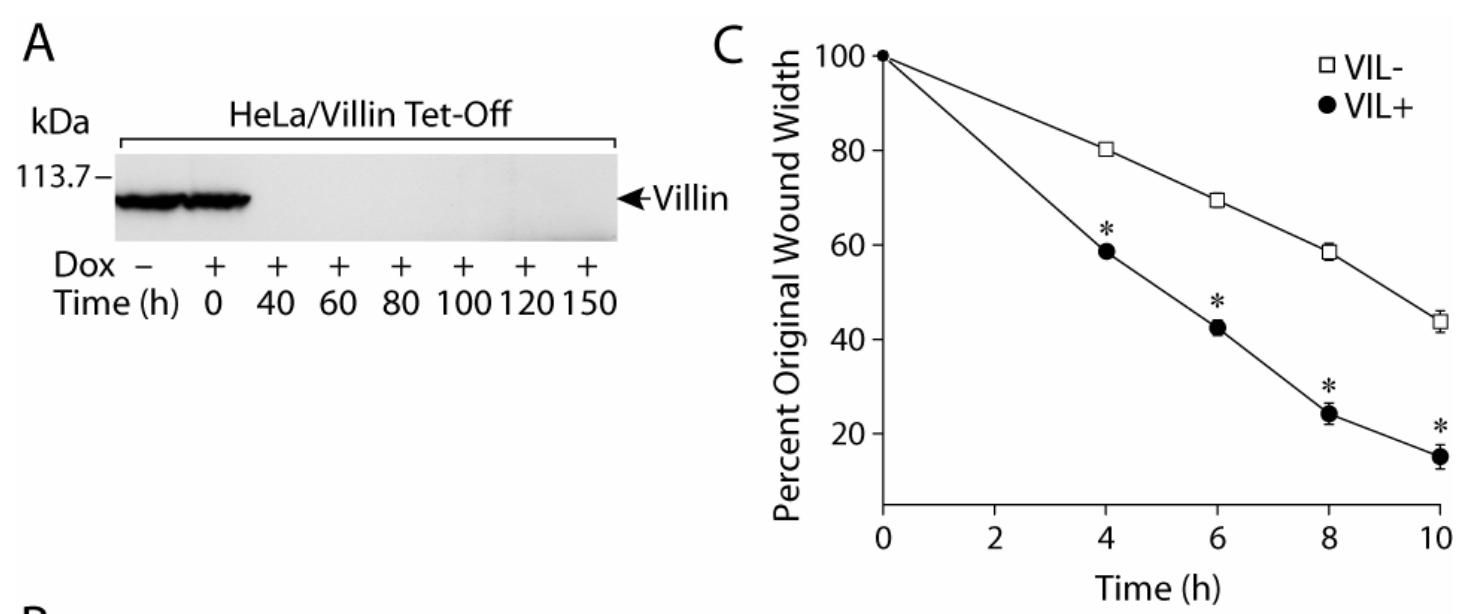

B
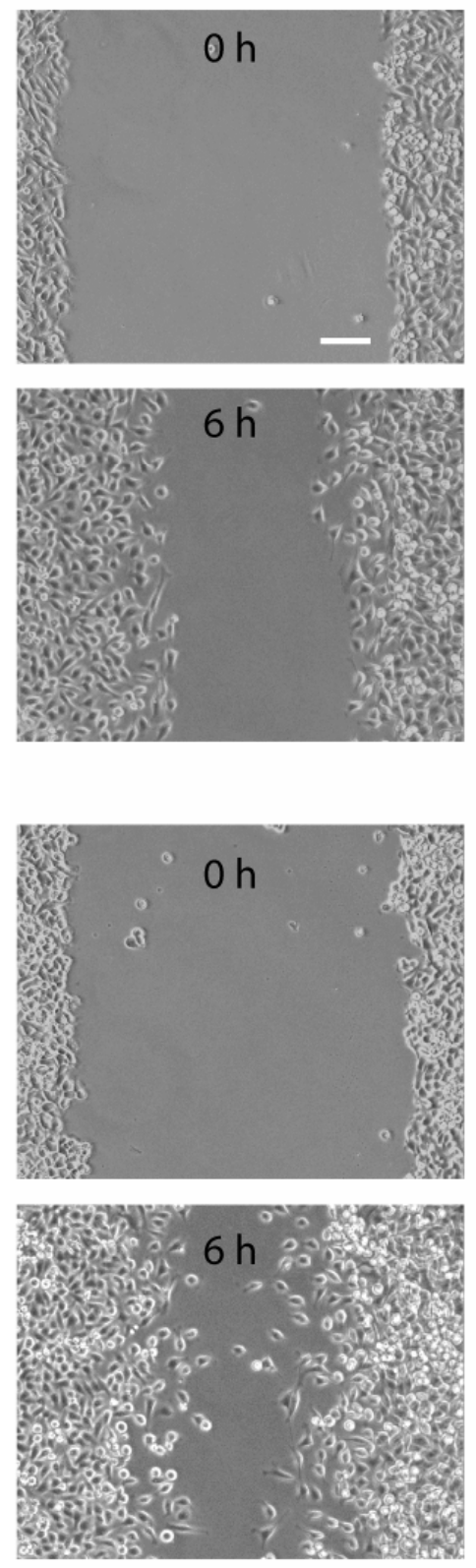

VIL/NULL
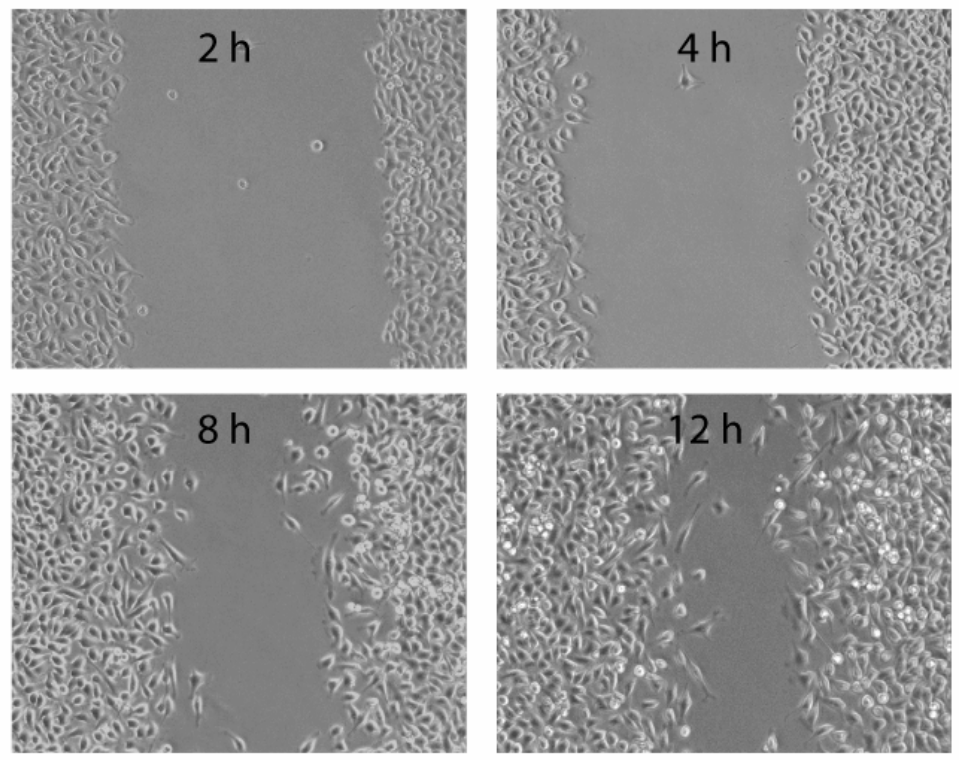

VIL/FL
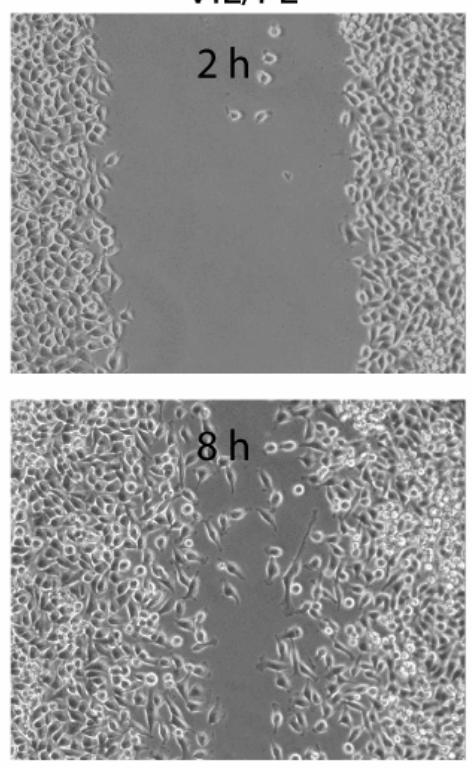
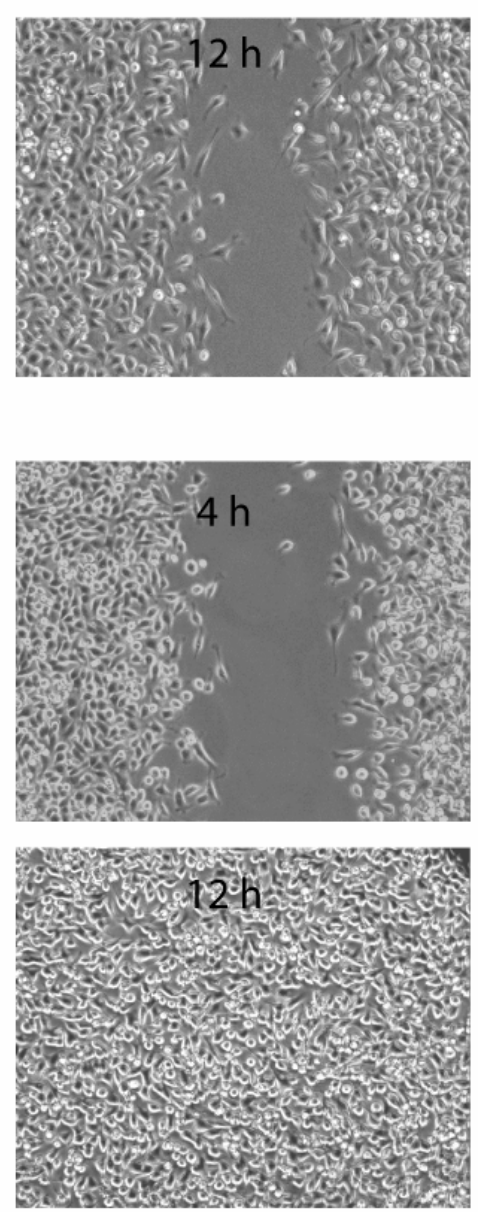
of doxycycline) to migrate into the empty wound space was observed. Assessment of cell migration revealed that wild-type villin expressing cells exhibited a dramatic increase in cell migration compared with villin-null cells (at $12 \mathrm{~h}$, four fold, $\mathrm{n}=24, \mathrm{p}<0.05$; figure $1 \mathrm{~B}$ ). Significant changes in the migratory capacity of villin-expressing cells were also observed at earlier time points (figure 2.1C). Based on these data, all subsequent migration studies were done between $0-10 \mathrm{~h}$ post-wounding. Untransfected HeLa cells as well as HeLa cells transfected with vector alone migrate at the same rate in the absence or presence of doxycycline (data not shown). Cell migration was studied at $10 \mathrm{~h}$ post-wounding and in the presence of $1.0 \%$ serum, conditions that do not favor cell proliferation. Nevertheless, DNA synthesis using 5-bromo-2'-deoxyuridine (BrdU) immunocytochemistry was measured and showed no significant difference in VIL/NULL and VIL/FL cells (figure A.1). These studies demonstrate that overexpression of human villin increases cell migration. Hepatocyte growth factor (HGF) is a multifunctional cytokine that can act as a motility inducing factor for epithelial cells through the c-met tyrosine kinase (Sonnenberg et al., 1993). Similarly EGF has been reported to enhance epithelial cell migration by mechanisms varied from HGF (Singh et al., 2004) as well as comparable to HGF (Muller et al., 2002). We measured the effects of both HGF and EGF on cell migration. VIL/NULL and VIL/FL cells were cultured in low serum (1.0\%) medium for $24 \mathrm{~h}$ prior to wounding. Cells were treated with HGF $(10 \mathrm{ng} / \mathrm{ml})$ or EGF (10 $\mathrm{nM}$ ) for 30 min and then wounded. The HGF and EGF treated cells were washed and the media was replaced with DMEM containing $1.0 \%$ serum. 
As shown in figure 2.2, at $6 \mathrm{~h}$ post-wounding in the absence of any treatment VIL/FL migrated faster than VIL/NULL cells $(\mathrm{n}=24, \mathrm{p}<0.05)$; treatment of these cells with HGF modestly enhanced the villin-induced cell migration $(n=24, p<0.05)$. This is comparable to previous reports demonstrating a small increase in response to HGF (Athman et al., 2003). Similarly treatment of VIL/FL cells with EGF enhanced villin-induced cell migration by about $9 \%(n=24$, $p<0.01)$. Similar data was obtained with cells cultured overnight in the absence of serum. Taken together these data show that expression of villin is sufficient to enhance cell migration and that HGF and EGF can modestly augment the villininduced increase in cell migration. Further it suggests that over expression of villin may have a direct effect on the signal transduction pathways that modify the actin cytoskeleton and enhance cell migration.

\subsubsection{Tyrosine phosphorylation of villin is essential for villin-induced cell}

migration. Previous studies from our laboratory have demonstrated that villin is a substrate in vitro for the src kinase(s) (Panebra et al., 2001; Zhai et al., 2002). In addition our studies have suggested that villin phosphorylation and the resulting changes in the actin rearrangements might serve to promote the migratory phenotype of epithelial cells (Panebra et al., 2001; Zhai et al., 2002). To confirm this, we examined the migratory capacity of cells stably expressing wild-type villin in the absence or presence of the src kinase inhibitor PP2 and its inactive analog PP3. In agreement with our previous results, pretreatment of VIL/FL with the src kinase inhibitor PP2 inhibits the villin-induced cell migration.

While PP2 inhibits both basal as well as EGF-induced cell migra- 


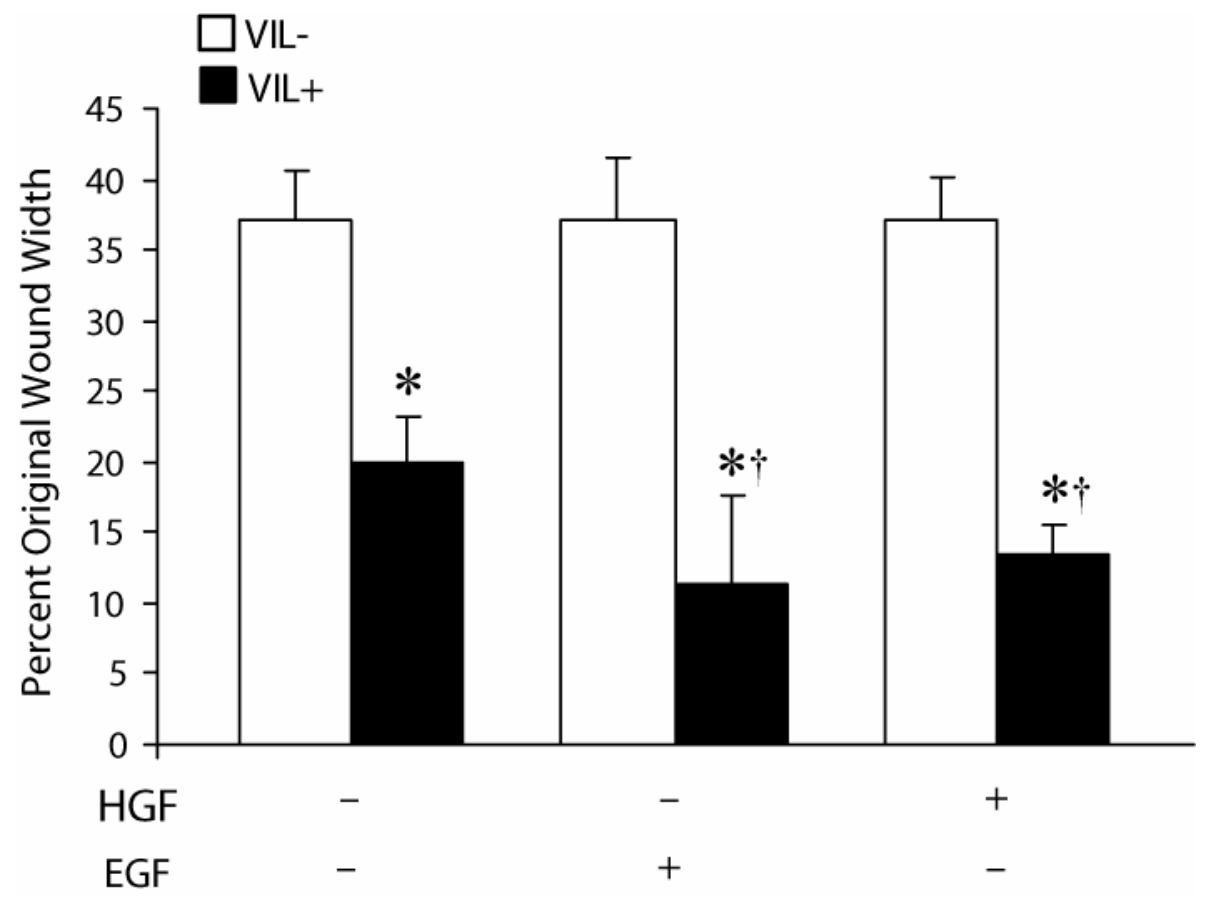

Figure 2.2 Villin-induced cell migration is enhanced by HGF and EGF. VIL/FL cells were treated without or with HGF $(10 \mathrm{ng} / \mathrm{ml})$ or EGF $(10 \mathrm{nM})$ for $30 \mathrm{~min}$ and cell migration followed over a period of $6 \mathrm{~h}$. Wound repair is expressed as a percentage of the initial wound area after $6 \mathrm{~h}$. VIL (-) refers to each clone cultured in the presence of doxycycline, whereas VIL $(+)$ refers to the same clone cultured in the absence of doxycycline. The error bars are the measured SEM, and the asterisk $\left({ }^{*}\right)$ and cross $(\stackrel{i}{)})$ denote statistically significant values $(p<0.05$, $\mathrm{n}=24$, compared with VIL $(-)$ cells $)$ and $(p<0.05, n=24$, compared with untreated cells) respectively. 
tion, the effects of PP2 on villin-induced cell migration is significantly higher $(11 \%$ in VIL/NULL cells versus $20 \%$ in VIL/FL cells, $n=24, p<0.05$ ) (figure $2.3 \mathrm{~A}$ ). Cells treated with the inactive analog PP3 behaved like untreated VIL/FL . A phospho-villin antibody (VP-70782) that specifically recognizes recombinant tyrosine phosphorylated villin (VILT/WT) and does not cross-react with recombinant nonphosphorylated villin protein (VIL/WT) (figure 2.3B upper panel) was used to immunoprecipitate tyrosine phosphorylated villin from HeLa cells. A commercially available anti-phosphotyrosine antibody (PY-20) confirms the specificity of the phospho villin antibody (figure 2.3B upper panel). The lower panel in figure 2.3B is an SDS-PAGE of VIL/WT and VILT/WT stained with GelCode Blue, showing the expression of the two recombinant proteins VILT/WT and VIL/WT. Consistent with our previous studies (Panebra et al., 2001; Zhai et al., 2002), tyrosine phosphorylation of villin (in response to wounding or treatment with HGF/EGF) is inhibited by PP2 but not PP3 (figure 2.3C). Using this approach, no basal tyrosine phosphorylation of villin in the absence of wounding or EGF/HGF treatment could be detected in these cells with either phospho-villin antibody (VP-70782) or phospho-tyrosine antibody (PY-20). Either there is no basal phosphorylation of villin in the absence of wounding or growth factor treatment, or there is some basal tyrosine phosphorylated villin but the levels are too low to be detected by western analysis. These data suggest that villin may be phosphorylated in vivo by src kinase(s) and that villin-induced motility may be mediated by activation of src kinase(s) and tyrosine phosphorylation of villin. To further confirm these observations, we used a 
Figure 2.3 Villin-induced cell migration is regulated by c-src kinase. (A) The Src kinase inhibitor PP2 inhibits villin-induced cell migration. VIL/FL cells were treated without or with the Src-kinase specific inhibitor PP2 (10 nM). The error bars are the measured SEM, and the asterisk ( $\left(^{*}\right)$ and cross ( $(t)$ denotes statistically significant values compared with VIL (-) cells and untreated cells $(p<$ $0.05, \mathrm{n}=24$ ) respectively. (B) Characterization of phospho-villin antibody. Recombinant phosphorylated (VILT/WT) and nonphosphorylated (VIL/WT) villin proteins were used to determine the specificity of the phospho-villin antibody. (C) HeLa cells cultured in the presence or absence of doxycycline were wounded (20 wounds/dish) and allowed to migrate in the presence of HGF $(10 \mathrm{ng} / \mathrm{ml})$ with or without the Src kinase inhibitor PP2 (10 nM) or its inactive analog PP3 (10 nM). (D) Expression of dominant negative c-src in VIL/NULL and VIL/FL cells. This is a western blot with c-src monoclonal antibodies. (E) Dominant negative c-src inhibits villin-induced cell migration. VIL/FL and VIL/NULL cells were infected with recombinant adenovirus [Ad-DN-c-src (dominant negative c-src)] for $4 \mathrm{~h}$ at a multiplicity of infection of 100 . This was followed by measurement of cell migration. (F) Dominant negative c-src inhibits the tyrosine phosphorylation of villin. Tyrosine phosphorylated villin was immunoprecipitated from VIL/FL cells infected with or without Ad-DN-c-src by using anti-phosphotyrosine antibody (clone PY-20), and western analysis was done using a villin mAb. This is a representative of three experiments with similar results. 
A

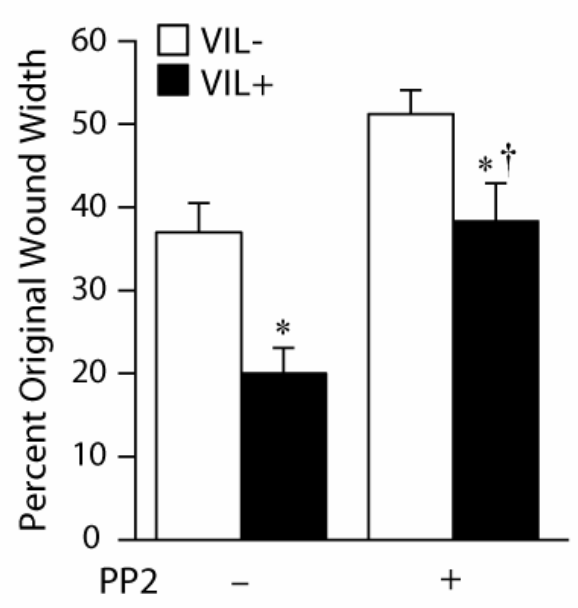

B

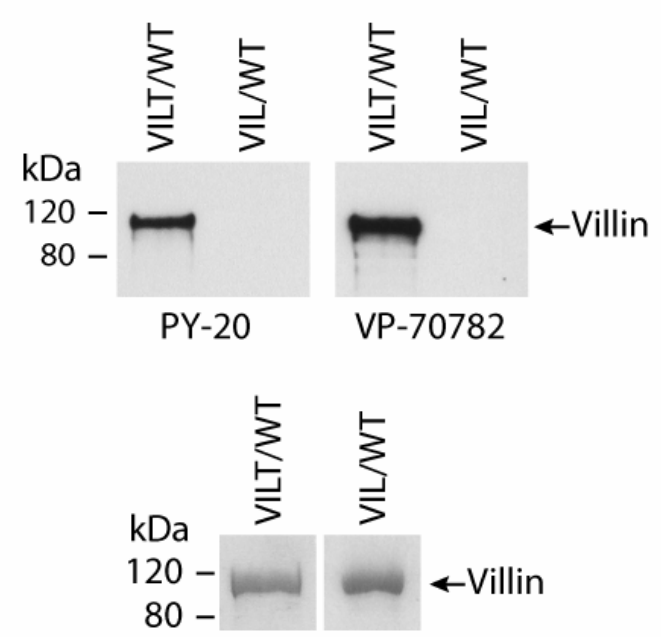

C $\mathrm{kDa}$
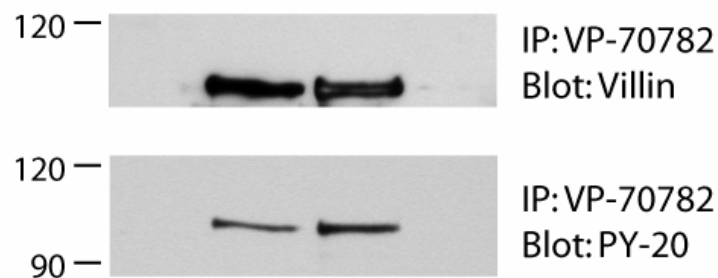

Wound/HGF $-\quad+\quad+\quad+$

$\mathrm{PP} 3$ - -+

$\mathrm{PP} 2$ - $\quad$ - $\quad$ -
D

$\mathrm{kDa}$

90 -

$60-5 \leftarrow$ C-srC

$45-$

AD-DN-cSrC + - + -

Villin ++--

E

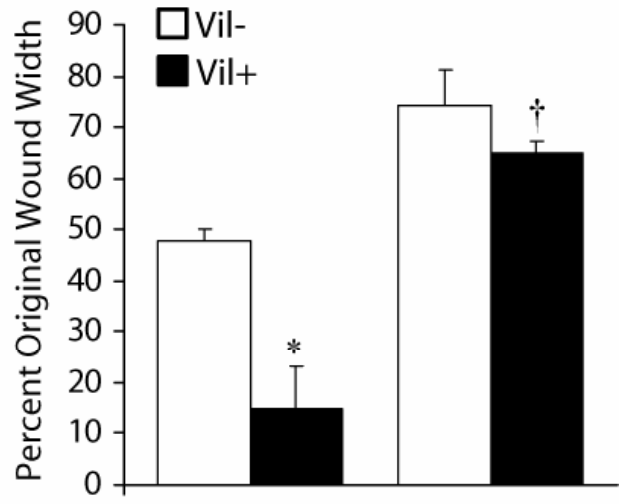

Ad-DN-c-src - +

$\mathrm{F}$

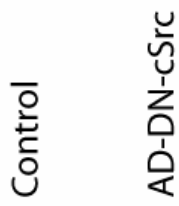

IP:VP-70782

Blot:PY-20

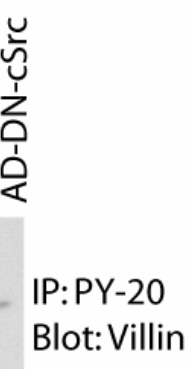


dominant negative construct of c-src (Ad-DN-c-src). HeLa cells expressing fulllength villin cultured in the absence or presence of doxycycline were infected with or without Ad-DN-c-src (figure 2.3D) and we measured the effect of expression of dominant negative c-src on villin-induced cell motility. A lower band around 57 $\mathrm{kDa}$ was seen in these western blots and was determined to be a degradation fragment of c-src (figure 2.3D). As shown in figure 2.3E, VIL/NULL and VIL/FL cells infected with Ad-DN-c-src migrate at very similar rates. Thus, expression of Ad-DN-c-src antagonized the capacity of villin to enhance cell motility, indicating that phosphorylation of villin by c-src is required for the villin induced increase of cell migration. Infection with an unrelated vector (Ad-EGFP) had no effect on cell migration (figure A.2). Over-expression of Ad-DN-c-src also inhibited the tyrosine phosphorylation levels of villin in VIL/FL cells (figure 2.3F). Ad-DN-C-src did not completely inhibit the tyrosine phosphorylation of villin unlike PP2. One possibility is that PP2 inhibits tyrosine phosphorylation of villin completely because PP2 can inhibit not only c-src but also other members of the Src family including c-yes and c-fyn, which may also be involved in the phosphorylation of villin.

\subsubsection{Tyrosine phosphorylation at $\mathrm{Y}-60, \mathrm{Y}-81$, and $\mathrm{Y}-256$ is essential for} villin-induced cell migration. Previous studies from our laboratory have demonstrated that c-src phosphorylates human villin on tyrosine $46,60,81$, and 256 (Zhai et al., 2002). To determine the role of tyrosine phosphorylated villin in cell migration, we examined the migratory capacity of cells stably expressing either wild-type or phosphorylation site mutants of villin, namely VIL/Y46F,VIL/Y60F, VIL/Y81, and VIL/Y256F. Multiple clones expressing the 
villin proteins were selected by immunoblotting using villin antibodies. Clones expressing similar amounts of villin proteins were selected for the migration studies (figure 2.4A). We assessed the effect of overexpression of wild-type and mutant villin proteins in HeLa Tet-Off cells on the organization of the actin cytoskeleton. HeLa Tet-Off cells expressing wild-type and mutant-villin proteins were stained with Alexa Phalloidin 568 (red) to examine the F-actin distribution and the localization of villin was examined using a villin monoclonal antibody (green) (figure $2.4 \mathrm{~B}$ and, figure A.3). The overall morphology of HeLa cells reconstituted with villin or with empty vector appears strikingly different. Phalloidin staining showed that actin stress fibers were lost in villin expressing HeLa cells (figure 2.4B, a-b). The transfection of HeLa cells with wild-type villin results in cells that are less flat and develop membrane ruffles concurrent with the reorganization of F-actin (figure 2.4B, b1-b3). Coincident with a virtual lack of stress fibers in these cells is the appearance of F-actin rich membrane ruffles on the dorsal surface and filopodia like structures, including microspikes on the ventral surface. All of these structures contained villin. This is consistent with previous work done by consistent with previous published reports (Friederich et al., 1989; Franck et al., 1990). The villin and F-actin in these cells localized at the cell perimeter where both proteins co-localized to peripheral ruffles, filopodia and microspikes (figure 2.4B, b1-b3). These results indicate that villin can direct the localization of F-actin to the cell cortex. The distribution of the villin mutant, Y46F, was similar to wild-type in that greater than $90 \%$ of the villin protein as well as the 
Figure 2.4 Tyrosine phosphorylation of villin is required for villin-induced increase in cell migration. (A) HeLa cells were stably transfected with wild-type (VIL/FL) and phosphorylation site mutants of villin, namely, Y46F, Y60F, Y81F, and Y256F. This figure shows representative clones of each villin construct transfected in HeLa cells. Data are representative of six experiments with similar results. (B) Villin expression results in reorganization of the actin cytoskeleton. HeLa cells transfected with wild-type (VIL/FL), and mutant villin proteins were analyzed by confocal microscopy. Double staining of villin $\left(a_{1}-f_{1}\right)$ and F-actin $\left(a_{2}-\right.$ $f_{2}$ ) was performed using villin monoclonal antibodies (1:100) and FITCconjugated anti-mouse IgG (1:200) and Alexa-Phalloidin 568 (1 $\mu \mathrm{g} / \mathrm{ml})$, respectively. Composite images of villin (green) and F-actin staining (red) are shown. Merged images (a3-f3) show colocalization of villin and F-actin. Bars, 3 $\mu m$. (C) HeLa cells expressing equal amounts of wild-type and phosphorylation site mutants ( $Y$ to $F$ ) of villin were used in wound-healing experiments. Wound repair is expressed as a percentage of the original wound width after $10 \mathrm{~h}$. The error bars are the measured SEM, and the asterisk $\left(^{*}\right)$ and cross $(\dagger)$ denote statistically significant values $(p<0.05, n=24$.). (D) Tyrosine phosphorylation of wild-type and mutant villin proteins. Cell extracts from VIL/FL and the mutant villin cell lines VIL/Y46F, VIL/Y60F, VIL/Y81F, and VIL/Y256F were immunoprecipitated with phospho-villin antibody (VP-70782) and western analysis done with villin mAb or phospho-tyrosine antibody (PY-20). This is not a quantitative western blot. The western blot is representative of three other experiments with similar results. 

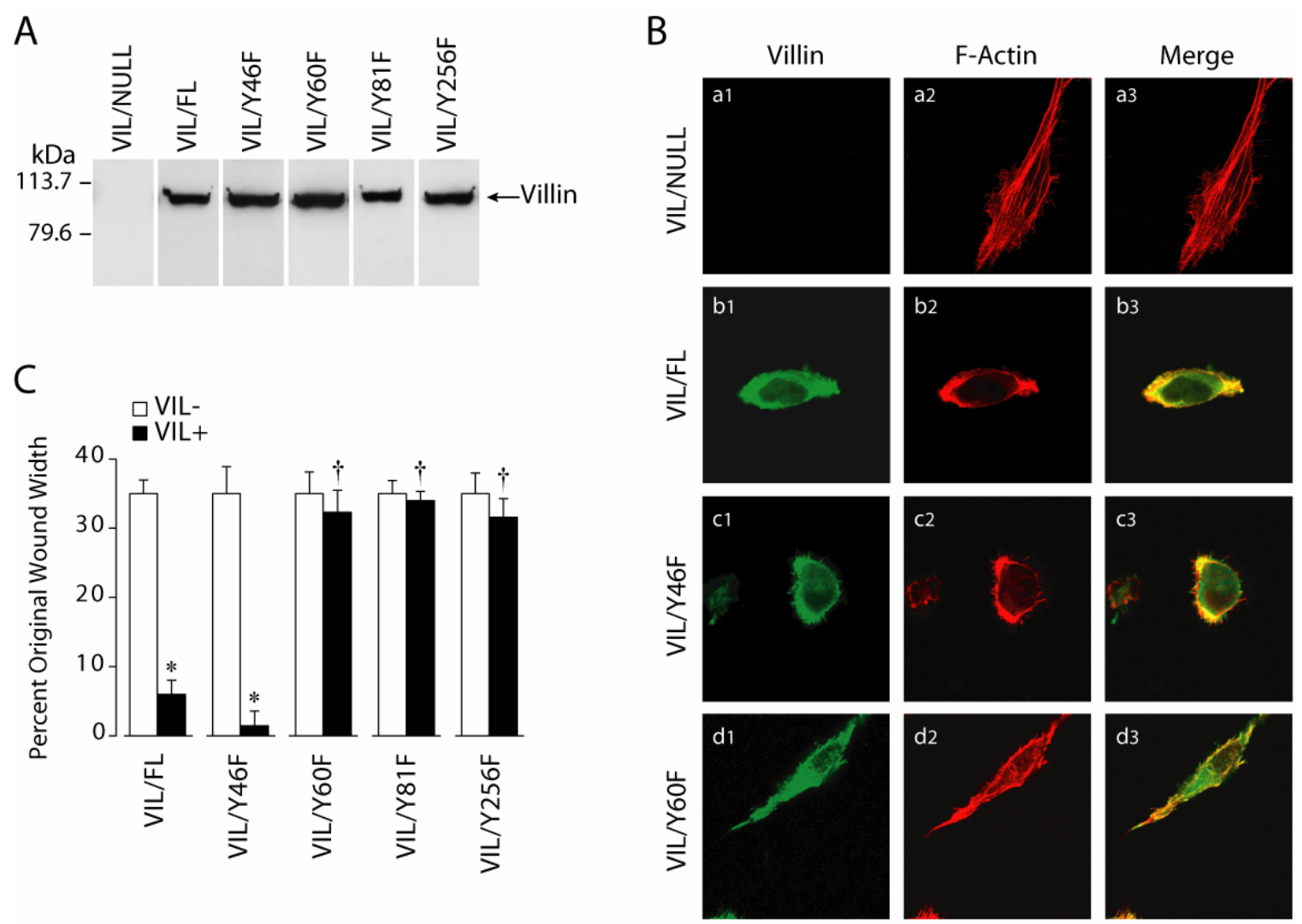

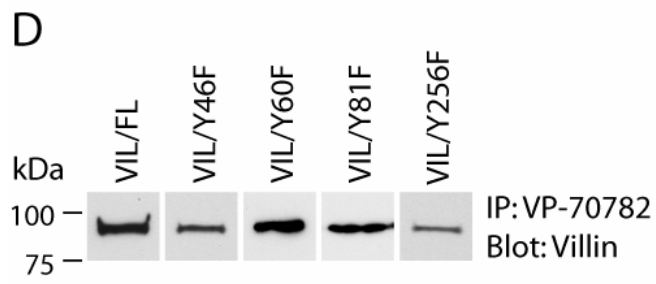
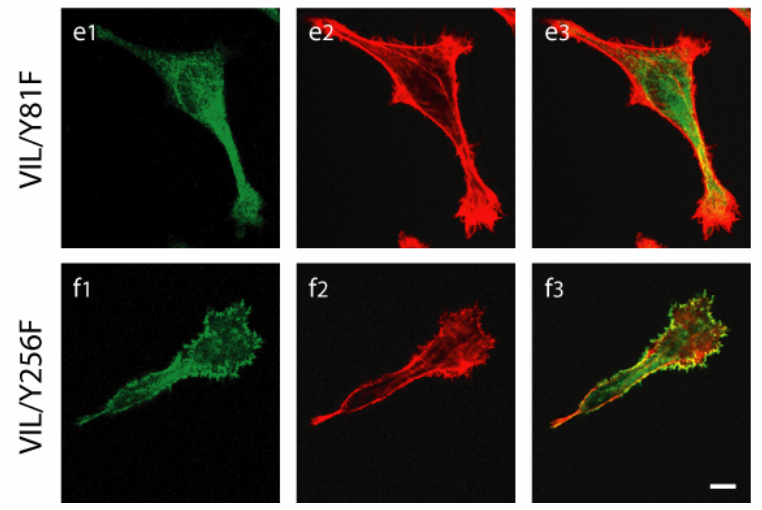
F-actin co-localized to the cell margin (figure 2.4B, c1-c3). The morphology of these cells was similar to VIL/FL cells with a disassembly of stress fibers and colocalization of the villin protein with F-actin in membrane ruffles, filopodia, and microspikes. In contrast, expression of villin phosphorylation mutants Y60F, Y81F or Y256F resulted in redistribution of $\mathrm{F}$-actin, suggesting that colocalization of villin and F-actin is dependent on tyrosine phosphorylation of villin at these sites (figure 2.4B, d-f). In all these mutant cell lines, the villin mutant proteins were distributed diffusely in the cytoplasm as well as at the cell periphery. In addition, the distribution of F-actin was altered and was associated not only with the cell surface structures but also intracellularly including what appear as dorsal arcs (figure 2.4B, e2 and f2). There was limited co-localization of villin and F-actin at the cell margins in these cells and the majority of the villin and F-actin remained segregated between the cell margins and the intracellular compartment. These data suggest that phosphorylatable tyrosine residues at $Y$ $-60,-81$, and -256 are required for both the colocalization of villin with F-actin as well as for the rearrangement of the actin cytoskeleton in the cell. Collectively, these results suggest that expression of villin is involved in reorganization of the actin cytoskeleton and further that villin-induced regulation of cell motility may be dependent on this redistribution of the actin cytoskeleton. There was no change in the total F-actin content in wild-type or mutant villin cell lines (figure A.4).

Our earlier studies including data presented in this study suggest that villin phosphorylation and the resulting changes in the actin rearrangements in the cell might serve to promote the migratory potential of epithelial cells. To confirm this, 
we examined the migratory capacity of cells stably expressing wild-type or phosphorylation-site mutants of villin. As shown in figure 2.4D, wild-type villin enhanced cell migration while the villin phosphorylation site mutants VIL/Y60F, $\mathrm{VIL} / \mathrm{Y} 81 \mathrm{~F}$, and VIL/Y256F were able to completely reverse the migratory phenotype of HeLa cells, reducing their migration rates to VIL/NULL cells. In contrast, the villin mutant VIL/Y46F behaved like wild-type villin suggesting that phosphorylation at this site is not essential for cell migration. To further quantify cell migration rate, we measured cell velocity. As shown in table 2.1, the average velocity $(0.13 \mu \mathrm{m} / \mathrm{min})$ of $\mathrm{VIL} / \mathrm{FL}$ and $\mathrm{VIL} / \mathrm{Y} 46 \mathrm{~F}$ shows an approximately $50 \%$ increase in cell velocity compared with the villin-null HeLa cells $10 \mathrm{~h}$ postwounding $(n=24, p<0.05)$. In contrast, the villin mutants VIL/Y60F, VIL/Y81F, and VIL/Y256F moved at a lower velocity, speeds similar to the villin-null HeLa cells $(0.09 \mu \mathrm{m} / \mathrm{min}, \mathrm{n}=48)$. There was no significant difference in cell proliferation between VIL/NULL, VIL/FL or cells expressing the phosphorylation site mutants of villin (figure A.1). The villin protein is tyrosine phosphorylated in all these cell lines in response to wounding. This is not a quantitative western blot since comparison of phosphorylation levels in different cell lines with a point mutation in one of the three phosphorylation sites could not be done with a great deal of confidence. These data confirm that tyrosine phosphorylation of villin regulates effective cell migration and further they characterize the specific tyrosine residues that control this process. To confirm the role of the specific tyrosine residues in the regulation of villin induced cell migration, we used phosphomimetics, namely tyrosine $(Y)$ to gluatamic acid $(E)$ phosphorylation site 
Table 2.1 Tyrosine phosphorylation of villin regulates the rate of cell migration.

\begin{tabular}{ll}
\hline $\begin{array}{c}\text { Villin } \\
\text { Construct }\end{array}$ & $\begin{array}{c}\text { Migration speed } \\
(\mu \mathrm{m} / \mathrm{min})\end{array}$ \\
\hline VIL/NULL & $0.090 \pm 0.002$ \\
VIL/FL & $0.130 \pm 0.002^{*}$ \\
VIL/Y46F & $0.136 \pm 0.003^{*}$ \\
VIL/Y60F & $0.094 \pm 0.002$ \\
VIL/Y81F & $0.091 \pm 0.001$ \\
VIL/Y256F & $0.095 \pm 0.001$ \\
\hline
\end{tabular}

The average velocity $(0.13 \mu \mathrm{m} / \mathrm{min})$ of $\mathrm{VIL} / \mathrm{FL}$ and $\mathrm{VIL} / \mathrm{Y} 46 \mathrm{~F}$ shows an $\sim 50 \%$ increase in cell velocity compared with the villin-null HeLa cells $10 \mathrm{~h}$ postwounding $(n=24, p<0.05)$. In contrast, the villin mutants $\mathrm{VIL} / \mathrm{Y} 60 \mathrm{~F}$, $\mathrm{VIL} / \mathrm{Y} 81 \mathrm{~F}$, and $\mathrm{VIL} / \mathrm{Y} 256 \mathrm{~F}$ moved at a lower velocity, speeds similar to the villinnull HeLa cells $(0.09 \mu \mathrm{m} / \mathrm{min}, \mathrm{n}<48)$. ${ }^{*}$ Denotes statistically significant values ( $\mathrm{P}$ $<0.05, \mathrm{n}=24)$. 
mutants of villin. Multiple clones expressing the phosphomimetic villin proteins were selected by immunoblotting using villin monoclonal antibodies. Clones expressing comparable amounts of villin proteins were selected for the migration studies (figure 2.5A). The intracellular distribution of villin as well as the F-actin localization was also examined using confocal microscopy (figure 2.5B and figure A.5). As shown in figure 2.5B, d-f, Y60E, Y81E, and Y256E look like VIL/FL. In all these cell lines, villin and F-actin co-localize at the cell margin and in the membrane ruffles. In contrast, VIL/Y46E resembles the VIL/NULL cells (figure 2.5B, c1-c3). As shown in figure 2.5C, VIL/Y60E, VIL/Y81E, and VIL/Y256E migrated faster than the comparable $\mathrm{Y}$ to $\mathrm{F}$ mutants (figure $2.4 \mathrm{C}$ ). At $10 \mathrm{~h}$ postwounding $95 \%$ of the wound was closed in cells expressing VIL/FL compared with villin-null cells ( $65 \%$ closed). The phosphomimetics behaved like VIL/FL in demonstrating enhanced cell migration compared with the villin-null cells, with $80-88 \%$ of the wound closed at $10 \mathrm{~h}$ post-wounding in VIL/Y256, VIL/Y81E, and VIL/Y60E respectively (figure $2.5 \mathrm{C}$ ). The rate of cell migration in VIL/Y60E, VIL/Y81E, and VIL/Y256E was not identical to that of VIL/FL but were dosedependent and densitometric analysis revealed that they paralleled the protein expression levels in these cell lines. The villin mutant VIL/Y46E in contrast inhibited cell migration, migrating at the same rate as villin-null cells, suggesting that this site must remain dephosphorylated for the motogenic effects of villin. Together, these results indicate that efficient cell migration from wounded cell monolayers requires tyrosine phosphorylation of villin. 
Figure 2.5 Phosphorylation of villin at $\mathrm{Y}-60, \mathrm{Y}-81$, and $\mathrm{Y}-256$ is required for villininduced cell migration. (A) HeLa cells were stably transfected with wild-type (VIL/FL) and phosphorylation site mutants of villin, namely, Y46E, Y60E, Y81E, and Y256E. This figure shows representative clones of each villin construct transfected in HeLa cells. Data are representative of six experiments with similar results. (B) Villin expression results in reorganization of the actin cytoskeleton. HeLa cells transfected with wild-type (VIL/FL) and mutant villin proteins were analyzed by confocal microscopy. Double staining of villin $\left(a_{1}-f_{1}\right)$ and F-actin $\left(a_{2}-\right.$ $f_{2}$ ) was performed using villin monoclonal antibodies (1:100) and FITC conjugated anti-mouse IgG (1:200) and Alexa-Phalloidin 568 (1 $\mu \mathrm{g} / \mathrm{ml})$, respectively. Composite images of villin (green) and F-actin staining (red) are shown. Merged images show colocalization of villin and F-actin (a3-f3). Wild-type villin and VIL/Y60E, VIL/Y81E, and VIL/Y256E colocalize with F-actin at the cell periphery. In contrast, phosphorylation mutants of villin VIL/Y46E shows intracellular distribution of villin and F-actin with minimal colocalization of villin and F-actin at the cell surface. Bars, $3 \mu \mathrm{m}$. (C) HeLa cells expressing equal amounts of wild-type and phosphorylation site mutants ( $Y$ to $E$ ) of villin were used in wound-healing experiments. Wound repair is expressed as a percentage of the original wound width at $10 \mathrm{~h}$. The error bars are the measured SEM, and the asterisk $\left(^{*}\right)$ and cross $(\dagger)$ denote statistically significant values $[p<0.05, n=$ 24, compared with VIL (-) cells and $p<0.05, n=24$, compared with untreated cells], respectively. Tyrosine phosphorylation of villin at Y60, Y81, and Y256 is a specific mediator of villin-regulated cell migration. 


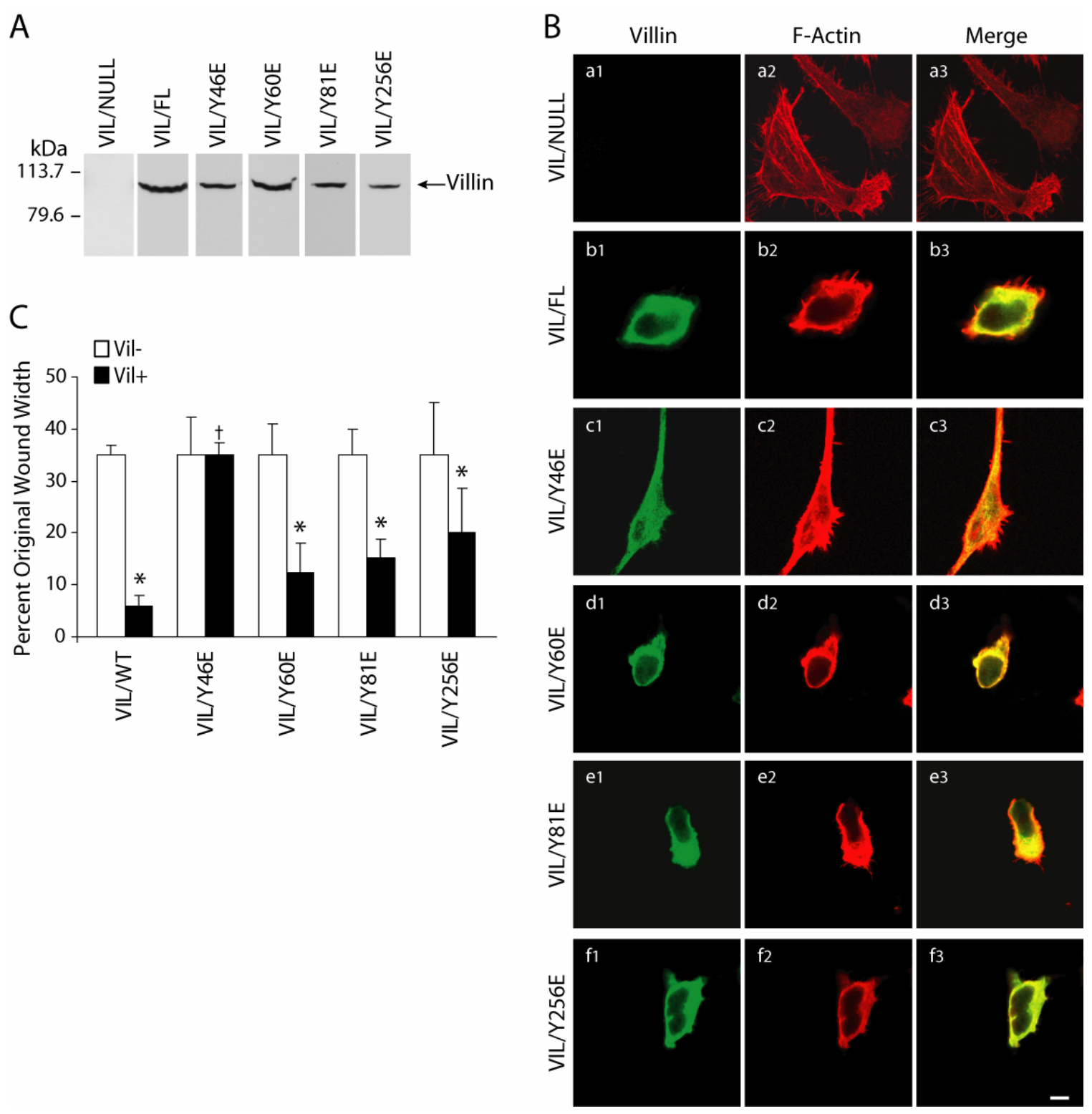




\subsection{Discussion}

Actin structures are dynamic and are affected by extracellular signals. While the organization of the actin cytoskeleton in a moving cell has been described reasonably well, it is still unclear how the cytoskeleton is directed by external signals to coordinate its activity to provide directional movement. In the present report we provide evidence that tyrosine phosphorylation of villin, which could occur in response to receptor activation or during wound healing results in both rearrangement of the microfilament structure as well as regulation of cell migration. In this study we further demonstrated that overexpression of villin enhances cell migration. This is consistent with previous observations made with proteins of the villin family including cofilin (Aizawa et al., 1996), CapG, and gelsolin (Sun et al., 1995). Data presented in our study suggest that an epithelial cell could also use villin as an intrinsic signal to stimulate actin assembly and increase cell migration in the absence of an external signal. Further, our studies demonstrate that this change in cell motility by villin can be enhanced in response to environmental cues such as chemo attractants. For our studies we used HGF, a cytokine that is known to coordinate changes in cell morphology associated with the induction of cell motility during epithelial-mesenchyme transition (Boyer et al., 1996) and EGF, another powerful motogen. Together these studies suggest that villin and other members of its family may function in vivo to provide a signaling mechanism for translating cell surface receptor mediated biochemical reactions into cell locomotion. 
Tyrosine kinase activity has been shown to be necessary for intestinal epithelial cell migration (Calalb et al., 1995; Cary et al., 1996; Parsons and Parsons, 1997; Reiske et al., 1999). Activation of c-src kinase has been reported in pre-neoplastic colonic adenomas and in colon carcinomas. In addition overexpression of pp60c-src has been demonstrated to increase the invasive behavior of intestinal epithelial cells (Pories et al., 1998). Thus, both tyrosine phosphorylation and activation of c-src kinase have been associated with the motile properties of intestinal epithelial cells. We have previously demonstrated that villin is phosphorylated in vitro by c-src kinase (Zhai et al., 2002). In the present study we provide evidence that in migrating cells, villin is a substrate for c-src kinase as well. Further, the potent and specific src kinase inhibitor PP2 inhibits the villin-induced increase in cell migration. Overexpression of the dominant negative c-src likewise inhibits villin-induced cell migration. These data suggest that villin is a substrate for src kinases in vivo just as it is in vitro and furthermore that src kinase phosphorylation of villin is important for villinmediated cell motility.

We have previously identified the tyrosine phosphorylation sites in villin and mapped phosphorylation at these sites with the actin modifying activities of villin (Zhai et al., 2002). Human villin contain four major tyrosine phosphorylation sites namely $\mathrm{Y}-46,-60,-81$, and -256 . In the current study we assessed the effects of tyrosine phosphorylation of villin as well as mapped the phosphorylation sites that regulate villin induced increase in cell migration. Our studies revealed that phosphorylation at $Y 60, Y 81$, and $Y 256$ are required for cell 
migration. Both Y46F and wild-type villin enhance cell migration and demonstrate similar intracellular distribution including redistribution of actin to the cell perimeter, loss of stress fibers and co-localization with F-actin, suggesting that actin cytoskeletal reorganization by villin is necessary for its role in cell migration. This is supported by the observation that the villin mutants VIL/Y60F, VIL/Y81F and VIL/Y256F migrate like the VIL/NULL cells and demonstrate a different subcellular distribution of villin as well as microfilament organization pattern. The most obvious difference between villin-induced migrating and villin-null or phosphorylation site mutant-villin cells is that villin that can be tyrosine phosphorylated at residues 60,81 , and 256 as well as the phosphomimetics localize at or near the cell surface. In contrast villin that cannot be tyrosine phosphorylated at these sites, show an intracellular distribution and are not very well distributed in cell surface structures. One possibility is that by altering the ability of villin to be tyrosine phosphorylated, we may have modified the ligandbinding properties of villin that determine its intracellular distribution and localization.

Recombinant tyrosine phosphorylated villin does not associate with $\mathrm{PIP}_{2}$, while non-phosphorylated villin does (Panebra et al., 2001). Further, we have demonstrated that tyrosine phosphorylation of villin decreases villin's binding affinity for F-actin (Zhai et al., 2001). This would suggest that within the cell at or near the leading edge, there could be two separate pools of villin, tyrosine phosphorylated villin that does not associate with the plasma membrane and has decreased affinity for F-actin and nonphosphorylated villin, which could bind both 
$\mathrm{PIP}_{2}$, and F-actin. Consistent with this observation we have previously reported that tyrosine phosphorylated villin redistributes to a Triton X-100-soluble fraction of intestinal villus cells, while non-phosphorylated villin remains associated with the F-actin filaments in a Triton-insoluble fraction (Khurana et al., 1997). Since phosphorylated villin does not associate with $\mathrm{PIP}_{2}$, we speculate that this Tritonsoluble pool may represent the cellular G-actin pool while the Triton X-100 insoluble pool is the F-actin pool in live cells. This is a reasonable speculation since we have also reported that tyrosine phosphorylation of villin releases an auto-inhibited conformation allowing it to sever actin filaments at physiological $\mathrm{Ca}^{2+}$ concentrations (Kumar and Khurana, 2004). Further, we have identified the biochemical properties of the individual phosphorylation sites in villin. Phosphorylation at $Y 60$ enhances the actin-severing activity of villin, suggesting that this site could be involved in generating new barbed ends (Zhai et al., 2002). In contrast, phosphorylation at Y81 and Y256 inhibits the ability of villin to polymerize actin filaments, thus altering the F-actin dynamics in vivo. These sites may have additional biochemical properties such as to decrease the binding affinity of villin for F-actin, decrease the actin-crosslinking activity of villin and/or induce conformational changes in villin resulting in constitutively-active villin that can sever actin filaments at nanomolar $\mathrm{Ca}^{2+}$. We have previously shown all these functions of villin to be regulated by tyrosine phosphorylation (Zhai et al., 2001; Kumar and Khurana, 2004). Alternatively, in vivo these tyrosine phosphorylation sites may be ligand-binding sites for second messengers that may regulate either tyrosine phosphorylation of villin such as c-src kinase or yet unidentified tyrosine 
phosphatases, as well as the SH2 domain of PLC- $\gamma_{1}$ (Panebra et al., 2001), all of which would modify villin-induced cell migration.

The integrity of these tyrosine residues is required for actin-nucleation and -depolymerization by villin, since phosphorylation of any one site impairs villin's capacity to nucleate actin, and likewise mutation at $Y 60$ to $F$ severely impairs phosphorylated villin's ability to sever actin filaments (Zhai et al., 2002). In other words, villin's ability to regulate the actin dynamics is dependent on its phosphorylation. Inhibition of cell migration by villin mutants suggests that a decrease in the actin-severing activity of villin is a negative regulator of villininduced increase in cell migration. In other words, increased actin depolymerization by villin could be the signal for the enhanced cell migration. The phosphomimetics lend further support to the idea that phosphorylation of villin at these three sites is necessary for the regulation of villin-induced migration. Interestingly the villin mutant Y46E inhibited cell migration. The significance of this observation is not clear at this point except it suggests that while phosphorylation at $\mathrm{Y} 46$ is not required for cell migration (VIL/Y46F behaves like VIL/FL), maintaining this site in a dephosphorylated form may be necessary for the villin-induced effects on cell migration. This also suggests that regulation of cell migration by villin may require both kinases and phosphatases maintaining the $\mathrm{Y}-60,-81$ and -256 sites in a phosphorylated state and the Y46 site in a dephosphorylated state for efficient cell migration. Based on our observations we propose the following model for villin-induced cell migration. We believe that spatially restricted accumulation of signaling molecules could determine the 
activation of one or more of villin's actin-regulatory properties, thus establishing villin's function in regulating cell motility. One of these signals could be a tyrosine kinase and/or phosphatase that could determine the phosphorylation state of villin. In the unphosphorylated state the actin-crosslinking property of villin could be important. For instance it is known that crosslinking of actin filaments is essential to convert the force of polymerization into forward movement of the membrane and the cell. While Arp $2 / 3$ is considered the major crosslinking component it is possible that in epithelial cells villin plays a significant role in crosslinking the actin filaments and generating stable lamellae. The crosslinking functions of villin could determine the rate and extent of lamellipodia formation and/or regulate the amount of F-actin incorporated into newly formed lamellipodia. Unphosphorylated villin could also bind the membrane phospholipid $\mathrm{PIP}_{2}$ and the villin-PIP 2 complex could favor persistent growth of the barbed ends by preventing capping of the barbed ends thus favoring both filament growth as well as allowing actin to push the membrane forward.

Phosphorylated villin could have other functions. Phosphorylation of villin could increase actin depolymerization, which could replenish the actin monomer pool thus allowing the cell to maintain high concentration of unpolymerized actin far from the equilibrium. Depolymerization of actin by villin could also produce new barbed ends that may be used for the formation of new filaments. Phosphorylation of villin and activation of villin's actin-capping function could help cap barbed ends. Capping of barbed ends could help maintain the length of actin filaments thus resulting in short filaments that can generate propulsive force and 
effectively push the membrane forward. Tyrosine phosphorylation of villin could regulate directed cell movement. It is possible that some of the phosphorylation site mutants exhibit lower migration rates because of lack of directed and coordinated movement. Since phosphorylated villin also disassembles actin filament bundles, cytoskeletal disassembly associated with disruption of actin bundles by tyrosine phosphorylated villin could generate pulling forces that may even be involved in the rearward retraction of a moving cell. Examples of such solationcontraction include microtubules that segregate chromosomes during mitosis (Mogilner and Oster, 2003), and sperm of the nematode Ascaris suum (Miao et al., 2003). Cell migration is intimately linked to cytoskeleton dynamics and our study demonstrates that tyrosine phosphorylation which affects cytoskeleton dynamics also affects cell migration. Our studies suggest that filament turnover in cells may be defined by the regulated action of actin-binding proteins interacting with signaling molecules. 


\section{Chapter 3}

\section{Interaction of phospholipase $\mathbf{C}-\gamma_{1}$ with villin regulates epithelial cell migration ${ }^{2}$}

2 Permission to reproduce by The American Society for Biochemistry and Molecular Biology. Tomar, A., George, S., Kansal, P., Wang, Y., and Khurana, S. (2006). Interaction of phospholipase $C-\gamma_{1}$ with villin regulates epithelial cell migration. J. Biol. Chem. 281, 31972-31986. 


\subsection{Introduction}

In intestinal epithelial cells as well as in the opossum kidney cell line, tyrosine phosphorylation of villin regulates ion transport (Khurana et al., 1997; Papakonstanti et al., 2000). In addition, rearrangement of the microvillar cytoskeleton and concomitant redistribution of villin have been reported in studies with enteroinvasive bacteria (Finlay and Cossart, 1997), in intestinal restitution (Nusrat et al., 1992; Albers et al., 1996), in renal ischemia, ischemia of the small intestine (Barthod, 1994; Golenhofen et al., 1995; Brown et al., 1997; White et al., 2000) as well as in colonic adenocarcinomas (Bacchi and Gown, 1991). It may be noted that villin expression is maintained in carcinomas derived from renal and intestinal epithelial cells. Villin is also expressed in other adenocarcinomas even though it is absent from normal tissue such as in Barrett's metaplasia and gastric cardia adenocarcinomas. More recently, chronic Helicobacter pylori infection has been shown to induce endogenous villin expression in the stomach (Rieder et al., 2005). Consistent with this and other studies, it has been suggested that villin expression arises in these tissues in response to chronic injury and may be a marker of pre-neoplastic lesions and may even participate in the altered genetic program that results in intestinal metaplasia (Tsukamoto et al., 2004). Studies done with the villin knock out mice show that villin is required for intestinal cell migration as well as Shigella infection (Athman et al., 2003; Athman et al., 2005). Abnormalities in villin gene 
expression have also been associated with progressive cholestatic liver disease (Phillips et al., 2003).

Villin is a member of a conserved family of actin-associated proteins widely expressed from slime molds to humans. Severin from Dictyostelium discoideum, fragmin from Physarum polycephalum and the vertebrate proteins villin, gelsolin, adseverin and scinderin belong to the group of actin-severing proteins that contain 3-6 repeats of a conserved domain. Villin contains six such domains (S1-S6). Like several proteins of its family, villin caps, nucleates and severs actin filaments and these functions are confined to the villin core (S1-S6). Villin contains in addition, a carboxyl-terminal domain (S7) called the headpiece, which provides villin with the ability to crosslink actin filaments (Glenney and Weber, 1981). We have previously demonstrated that villin is tyrosine phosphorylated in vitro and in vivo by c-src kinase (Panebra et al., 2001; Tomar et al., 2004; Wang et al., Submitted). Likewise, gelsolin, fragmin and CapG have been shown to be tyrosine phosphorylated in vitro by c-src (De Corte et al., 1997). Further, proteomic analysis of phosphotyrosyl-proteins in human lumbar cerebrospinal fluid has been shown to include tyrosine phosphorylated gelsolin (Yuan and Desiderio, 2003). These reports and our own data point to a more general mechanism involving tyrosine phosphorylation of this family of proteins. Thus, giving new properties to these proteins and adding another level of regulation that will be recognized by future studies involving the identification of the phosphorylated tyrosine residues and functional assays. Epithelial cells of the intestine and kidney express more than one protein of this family (villin, 
gelsolin and adseverin) (Lueck et al., 1998). We and others have previously reported that while these proteins share structural homology they are not functionally identical (Finidori et al., 1992; Panebra et al., 2001; Kumar and Khurana, 2004; Kumar et al., 2004a; Kumar et al., 2004b). The identification of the tyrosine phosphorylation sites and their molecular characterization in these proteins will facilitate our understanding of their functional diversity. Further, such studies will help elucidate why some cells express more than one protein of this family and whether these proteins have identical, overlapping or distinct functions in these tissues.

Tyrosine phosphorylation of villin releases its autoinhibited conformation allowing it to sever actin at physiologically relevant calcium concentrations (Kumar and Khurana, 2004). We have also reported that tyrosine phosphorylation regulates villin functions, specifically, villin's ability to modify the actin cytoskeleton, redistribution of F-actin in cells as well as villin-induced changes in cell shape and cell motility (Tomar et al., 2004). In addition, tyrosine phosphorylation of villin modifies villin's ligand-binding properties, including its association with phosphatidylinositol 4,5-bisphosphate $\left(\mathrm{PIP}_{2}\right)$, phospholipase C$\gamma_{1}$ (PLC- $\gamma_{1}$ ) and F-actin (Khurana et al., 1997; Panebra et al., 2001; Zhai et al., 2001). Regulation of villin's functions by tyrosine phosphorylation, which is often a consequence of receptor activation, suggests that villin may function to communicate cell surface activation to the cytoskeletal machinery.

In an effort to comprehend the role of tyrosine phosphorylation to villin's function, we have elected to identify the tyrosine phosphorylation sites in human 
villin and map villin's functions to these sites. We have previously identified four major phosphorylated tyrosine residues in the villin core (Zhai et al., 2002; Tomar et al., 2004). In the present study we identify six additional sites in the carboxylterminal villin core (domains S3-S6). With this study we have identified all the tyrosine residues in human villin that can be phosphorylated and mapped the functions of villin regulated by these phosphorylation sites. Specifically, we have characterized the role of these sites in regulating F-actin reorganization, cell morphology and cell motility by transfecting full-length, truncation or phosphorylation site mutants of villin in the tetracycline regulated HeLa and MDCK cells. In this report, we demonstrate for the first time that the carboxylterminal tyrosine phosphorylation sites in villin are required for its association with PLC- $\gamma_{1}$, thus determining their significance in villin-induced cell migration. Since there is considerable structural and functional homology between villin and other proteins of its family, the results presented herein help understand the relationship of phosphorylation with the role of these proteins in cell migration (Arpin et al., 1988; Arora and McCulloch, 1996; Folger et al., 1999; Athman et al., 2003; Tomar et al., 2004).

\subsection{Materials}

Epicurian coli TKX1 and BL21 competent cells and the Quick-Change sitedirected mutagenesis kit were from Stratagene. Glutathione Sepharose 4B Fastflow was from Amersham-Pharmacia. GelCode Blue was from Pierce. Monoclonal antibodies to phosphotyrosine (clone PY-20) were from ICN; 
monoclonal antibodies to villin were from Transduction Laboratories and, polyclonal phospho-villin antibody VP-70782 used in these studies has been described previously (Tomar et al., 2004). Monoclonal antibodies to phospholipase $\mathrm{C}-\gamma_{1}$ were purchased from Upstate Biotechnology. Monoclonal antibody to the influenza $A$ virus haemagglutinin $(\mathrm{HA})$ tag was purchased from Roche. Alexa Fluor 488-phalloidin was purchased from Molecular Probes; and Cy3-conjugated affinity purified donkey anti-mouse IgG was purchased from Jackson ImmunoResearch Laboratories. Lysophosphatidic acid (LPA) and epidermal growth factor (EGF) were purchased from Sigma-Aldrich. In situ Cell Death Detection kit, POD was purchased from Roche. HOECHST 33258 was purchased from Sigma-Aldrich. HeLa Tet-Off cells stably expressing the tTA tetracycline-controlled transactivator, G418, hygromycin, doxycycline, the eukaryotic expression vectors pTRE-6 x HN, pTRE-HA and the selection vector pTK-Hyg were purchased from BD Biosciences Clonetech. MDCK Tet-Off cells were a kind gift from Dr. Keith Mostov (UCSF, California). Lipofectamine ${ }^{\mathrm{TM}} 2000$ was purchased from Invitrogen Laboratories. $35 \mathrm{~mm}$ glass bottom culture dishes were purchased from Mat Tek Corporation. The muscle actin polymerization kit was purchased from Cytoskeleton (Denver, CO). All other chemicals were from Sigma or Invitrogen. Monoclonal antibodies to phospholipase C- $\gamma_{1}$ were purchased from Upstate Biotechnology. Monoclonal antibody to the influenza A virus haemagglutinin (HA) tag was purchased from Roche. Alexa Fluor 488phalloidin was purchased from Molecular Probes; and Cy3-conjugated affinity 
purified donkey anti-mouse IgG was purchased from Jackson ImmunoResearch Laboratories.

\subsection{Methods}

3.3.1 Tyrosine phosphorylation of villin in TKX1 cells. Full-length or mutant villin cDNA cloned in pGEX-4T1 were expressed in E. coli TKX1 cells as described previously (Panebra et al., 2001). Briefly, TKX1 cells carry a plasmid with the elk tyrosine kinase (tk) gene controlled by the trp promoter. The TKX1 cells were transformed with wild-type or mutant villin plasmids. A two-step protocol was followed to first induce the villin gene (by addition of isopropy- $\gamma$-Dthiogalactropyranosie (IPTG)) followed by induction of the tk gene (by addition of indoleacrylic acid (IAA)), which generated glutathione S-transferase (Gst)-tagged tyrosine-phosphorylated villin protein(s). TKX1 cells transformed with the villin gene and cultured in the absence of IAA were used to obtain non-phosphorylated villin controls. Tyrosine phosphorylated proteins were detected by western analysis using a phosphotyrosine monoclonal antibody (PY-20). Densitometric analysis was carried out using Scion Image software.

3.3.2 Amino-terminal truncation mutants of villin. To identify the villin phosphorylation site(s) we created amino-terminal truncation mutants of villin using full-length human villin cDNA cloned in the prokaryotic expression vector pGEX-4T1. Briefly, polymerase chain reaction was used to introduce EcoRI 
(Forward) and Xhol (Reverse) restriction sites using the following primers: CT, 5' (Forward)-AGGGAATTCGCCACACGGCCACTGACA, 5' (Reverse)-CTTTCTCGAGGAAT-AGGTACATTATTA;

CT1, 5' (Forward)-GTGGAATTCGTGAAGTTCGATGCCACA, 5'(Reverse)-CTTTCTCGAGGAAT-AGGTACATTATTA;

CT2,5' (Forward)-TTT GAATTCCCAGCGCGGGCCAATTTC, 5'(Reverse)- CTTTCTCGAGGAATAG-GTACATTATTA;

CT3, 5'(Forward)- AGGGAATTCGCCACACGGCCACTGACA, 5'(Reverse)- TTTGGCCㄷCGAGC-CCACAGTGTG;

CT4, 5'(Forward)- GTGGAATTCGTGAAGTTCGATGCCACA, 5' (Reverse)-TGGACCTCGAGCAGC-CGTGTGGAG.

The PCR amplicons were digested using EcoRI and Xhol and directionally cloned in pGEX-4T1. The cloning of the specific truncation mutants were confirmed by sequencing.

\subsubsection{Substitution of tyrosine with phenylalanine in villin truncation}

mutants. The putative phosphoryable tyrosine $(Y)$ residues in the truncation mutants (CT2-CT6) were changed to phenylalanine $(F)$ by designing complementary primers in which a $\mathrm{Y}$ codon was replaced with an $\mathrm{F}$ codon. Tyrosine's at positions $286,296,324,422,427,431,433,441,444,461,470$, $555,604,681,725$ were replaced with phenylalanine using the QuikChange sitedirected mutagenesis kit to make single-base change from TAT and TAC to TTT and TTC respectively. The mutation primers were as follows: Y286F, 5'(Forward)-TCACGAGGACTGGTTTCA-TCCTGGACCAGG and 5' (Reverse)- 
CCTGGTCCAGGATGAAACAGTCCTCGTGA; Y296F, 5' (Forward)-GGCCTGAAGATCITCGTGTGGAAAGGG and 5' (Reverse)-CCCTTTCCACACGAACATC-TTCAGGCC;

Y324F, 5' (Forward)-ATCAAAGCCAAGCAGTTCCCACCAAGCACACCAG and 5' (Reverse)-CTGTGTG-CTTGGTGGGAACTGCTTGGCTTTGAT;

Y422F 5' (Forward)-CTAGGCCACTTC-TTTGGGGGCGACTGC and 5' (Reverse)-GCAGTCGCCCCCAAAGAAGTGGCCT-AG;

Y427F, 5' (Forward)-GGGGGCGAC-TGCTTCCTGCTGCTCTAC and 5' (Reverse)-GTAGAGCAGCAGGAAGCA-GCAGTCGCCCCC;

Y431F, 5' (Forward)-TACCTGCTGCTCTTCACCTACCTCATC and 5' (Reverse)-GATGAGGTAGGTGA-AGAGCAGCAGGTA; Y433F, 5' (Forward)-CTGCTCTACACCTTCCTCA-TCGGCGAG and 5' (Reverse)-CTCG-CCGATGAGGAAGGTGTAGAGCAG;

Y441F, 5'(Forward)-GGCGAGAAGCA-GCATTTCCTGCTCTACGTTTGG and 5' (Reverse)-CCAAACGTAGAGCAGGAA-ATGCT-GCTTCTCGCC;

Y444F, 5' (Forward)-CATTACCTGCTCTTCGTTTG-GCAGGGC and 5' (Reverse)-GCCCTGC-CAAACGAAGAGCAGGTAATG; Y461F 5' (Forward)-ACAGCATCAGCTTTCA-AGCCGTCATC and 5' (Reverse)-GATGACGGCTTGAAAAGCTGATGCTGT; Y470F,5'(Forward)-CCTGGACATCCGGACCAGAAGTTCAATGGTGA and 5'(Reverse)-GACTGGTT-CACCATTGAACTTCTGGTCCAGGAT; Y555F, 5' (Forward)-ACCCAGTCTTGCT-GCTTTCTATGGTGTGGGAAG and 5' (Reverse)-CTTCCCACACCATAGAAA-GCAGCAAGACTGGGT; 
Y604F, 5' (Forward)-TGGGAAGGCCCCCITTGCC-AACACCAAGAG and 5' (Reverse)-CTCTTGGTGTTGGCAAAGGGGGCCTTCCCAC;

Y681F, 5' (Forward)-ACCACTG-CACAGGAATTCCTCAAGACCCATCCC and 5' (Reverse)-GGGATGGGTCTTGA-GGAATTCCTGTGCAGTGGT;

Y725F, 5' (Forward)-TAACACCAAATCCTTTGA-GGACCTGAAGG and 5' (Reverse)-CCTT-CAGGTCCTCAAAGGATTTGGTGTTA. A villin mutant lacking all ten identified tyrosine phosphorylation sites namely Y-46, -60, -81, $-256,-286,-324,-461,-555,-604$ and -725 (substituted with phenylalanine) was made to confirm the tyrosine phosphorylation status of recombinant villin expressed in TKX1 cells (VILT/WT (AYFM)).

3.3.4 Urea denaturation assay. To determine the effects of specific mutations on the overall stability of the villin molecules, fluorescence-monitored urea denaturation was performed on each recombinant protein as described previously (Kumar et al., 2004b). Fluorescence measurements were taken at an excitation wavelength of $280 \mathrm{~nm}$ and at an emission scan was performed from $335 \mathrm{~nm}$ to $360 \mathrm{~nm}$.

\subsubsection{Measurement of actin-polymerization and -depolymerization by} phosphorylated wild-type and point mutants of villin. The kinetics of actinpolymerization were determined using a muscle actin polymerization kit according to the instructions of the manufacturer and as described previously (Kumar et al., 2004a). The ability of villin to nucleate actin assembly or to depolymerize actin filaments was determined by its effect on the rate and extent 
of increase or decrease, respectively, of fluorescence of pyrene-labeled actin. Fluorescence measurements were performed at $25^{\circ} \mathrm{C}$ using the FluoroMax 3 spectrofluorometer. The excitation wavelength was set at $365 \mathrm{~nm}$, and the emission wavelength was set at $407 \mathrm{~nm}$.

\subsubsection{Measurement of actin uncapping by phosphorylated wild-type and} point mutants of villin. The actin-capping activity of wild-type and mutant villin proteins was measured essentially as described by Northrop et al using pyrenelabeled actin as described by Schafer et al (Northrop et al., 1986b; Schafer et al., 1996). $290 \mathrm{nM}$ villin-actin seed were used as nuclei for polymerization with pyrene-labeled G-actin $(1.4 \mu \mathrm{M})$ in a reaction volume of $200 \mu \mathrm{l}$. The increase in fluorescence was measured over time as described before (Kumar et al., 2004a). The concentration of calcium $(2.5 \mu \mathrm{M})$ used in the assays has been shown to be saturating for capping but not severing of actin filaments by villin.

\subsubsection{Transfection of HeLa and MDCK Tet-Off cells with full-length and} mutant villin cDNA. Full-length villin and the point mutants of villin (Y286F; Y324F; Y461F; Y555F; Y604F and Y725F were cloned in the eukaryotic expression vector pTRE-6 $\times$ HN by amplification using polymerase chain reaction of the coding sequences cloned in pGEX-4T1 as described before (Tomar et al., 2004). Three additional constructs were made: (i) VIL/ANFM, in which all four amino-terminal tyrosine phosphorylation sites, namely Y46, Y60, Y81 and Y256, were mutated to phenylalanine; (ii) VIL/ACFM, in which all six carboxyl-terminal tyrosine phosphorylation sites, namely Y286, Y324, Y461, Y555, Y604 and Y725 
were mutated to phenylalanine and (iii)VIL/AYFM, in which all ten phosphoryable tyrosine residues were mutated to phenylalanine. Yellow fluorescent protein tagged version of full-length villin was made by using superenhanced yellow fluorescent protein (SEYFP) subcloned into the Sal 1 site of full-length villin cloned into pTRE-HA between the HA tag and villin (SEYFP/FL) in collaboration with Dr. Ian Macara (University of Virginia School of Medicine, Charlottesville, VA) (Tomar et al., 2004). To check if villin and SEYFP were in frame the following primer was used, 5'-CATGGTCCTGCTGGAGTTCGTCA; and to check if SEYFP was in frame with the HA tag, the following sequencing primer was used, 5'-CGC-CTCCAGACGCCATCCACGCT. Deletion mutant S1-S3 (SEYFP/S1-S3) was made by designing complementary primers to introduce a stop codon at positions 338 of SEYFP/VIL using the QuikChange site-directed mutagenesis kit, as recommended by the manufacturer. The introduction of the stop codon was verified by sequencing. SEYFP tagged versions of VIL/ANFM, VIL/ACFM and VIL/AYFM were cloned by digesting VIL/ANFM, VIL/ACFM and VIL/AYFM cloned in PTRE-HA with Sal I. Likewise the $0.7 \mathrm{~kb}$ SEYFP fragment was obtained by digestion of SEYPF/FL with Sal I. The recombinant constructs VIL/ANFM, VIL/ACFM or VIL/AYFM were ligated with the SEYFP fragment using non-directional sticky end cloning. The cloning of the SEYFP insert was verified by sequencing and the SEYFP fragment was found upstream of VIL/ANFM, VIL/ACFM as well as VIL/AYFM sequence and in correct reading frame with the HA tag. HeLa Tet-Off cells were stably cotransfected with the wild-type or mutant villin plasmids and a selection plasmid 
carrying the hygromycin resistance gene. Villin-null HeLa and MDCK Tet-Off cells were transiently transfected with SEYFP/FL, SEYFP/ACFM or SEYFP/S1S3 plasmids and cells were analyzed $16-24 \mathrm{~h}$ post-transfection. Alternatively, MDCK Tet-Off cells were stably transfected with SEYFP/FL, SEYFP/ANFM, SEYPF/ACFM or SEYFPIAYFM.

3.3.8 Cell motility assay. HeLa Tet-Off cells transfected with wild-type or mutant villin proteins were seeded in six-well plates and cultured in the absence $(\mathrm{VIL}+)$ or presence (VIL -) of doxycycline. Confluent monolayers were scraped with a plastic pipette tip to generate wounds essentially as described before (Tomar et al., 2004). Images were obtained at the initial time of wounding and at various time intervals up to $24 \mathrm{~h}$ post-wounding. Data are expressed as a percentage of original wound width. Wound width measurements were averaged from two regions of the same well and the mean treated as a single data point. Comparisons between mean values were made using one-way repeatedmeasures analysis of variance and Tukey's modified t test (Benferroni criteria) with $p<0.05$.

3.3.9 Immunofluorescence microscopy. HeLa Tet-Off cells expressing wildtype and mutant villin proteins were cultured on coverslips and fixed in $3.7 \%$ formaldehyde and permeabilized by incubation in phosphate buffered saline containing $0.2 \%$ Triton $\mathrm{X}-100$ and $0.5 \%$ normal goat serum. Cells were incubated with villin monoclonal antibody $(1: 100)$ and Alexa Fluor 488-phalloidin was included to record the distribution of F-actin. The secondary antibody was 
Cy3-conjugated affinity purified donkey anti-mouse IgG (1:100). The fluorescence was examined by confocal laser scanning microscopy (LSM 5 PASCAL; Carl Zeiss, Thornwood, NY).

For time-lapse microscopy, cells were incubated on 35-mm glass bottom dishes. MDCK/HeLa cells transiently transfected with SEYFP/FL or SEYFP/S1S3 were used for these studies, 16-24 h post-transfection. Alternatively MDCK Tet-Off cells stably transfected with SEYPF/FL, SEYFP/ANFM or SEYFP/ACFM were used for these studies. Cells were washed twice with phosphate-buffered saline and $50 \mathrm{ng} / \mathrm{ml}$ of epidermal growth factor (EGF) or lysophosphatidic acid (LPA $1 \mu \mathrm{M}$ ) were added to the cells 5 min before time lapse images were captured. Time lapse images were acquired with a $40 \times$ objective on a confocal microscope (LSM 5 PASCAL, Carl Zeiss, and Thornwood, NY). Images were captured every 2 min for a maximum of 60 minutes.

\subsubsection{Measurement of lamellipodial protrusion rate. Lamellipodia were} characterized as thin regions 3-10 microns wide located at the cell margin. Using time lapse imaging, lamellipodia protrusion rate was measured as the increase in total cell area after growth factor (LPA $(1 \mu \mathrm{M})$ or EGF $(50 \mathrm{ng} / \mathrm{ml}))$ stimulated cell migration using time lapse images essentially as described before (Rottner et al., 1999; Athman et al., 2003). The mean relative area was plotted as a function of time to measure rate of protrusive activity after EGF/LPA treatment. Approximately 10 cells were examined and for all cells the rate remained approximately constant during the 20 minutes of observation. 
3.3.11 Immunoprecipitation and western blot analysis. HeLa or MDCK TetOff cells transfected with full-length villin (VIL/FL) or phosphorylation site mutants of villin namely, VIL/ACFM or VIL/AYFM were extracted with a solution containing 1\% Triton $\mathrm{X}-100,20 \mathrm{mM}$ HEPES, $\mathrm{pH} 7.2,150 \mathrm{mM} \mathrm{NaCl}, 1 \mathrm{mM}$ sodium orthovanadate, $50 \mathrm{mM} \mathrm{NaF}$ and a cocktail of protease inhibitors for 15 min at $4^{\circ} \mathrm{C}$. PLC- $\gamma_{1}$ were immunoprecipitated from the soluble extracts as described previously (Khurana et al., 1997) by using monoclonal antibodies to PLC- $\gamma_{1}$. Tyrosine phosphorylated proteins were Immunoprecipitated from MDCK Tet-Off cells lysed using modified RIPA buffer containing 50mM Tris- $\mathrm{HCl}$ pH 7.4, 1\% NP-40, 0.25\% Na-deoxycholate, $150 \mathrm{mM} \mathrm{NaCl}, 1 \mathrm{mM}$ EDTA, 1 mM PMSF, Aprotinin, leupeptin, pepstatin $1 \mu \mathrm{g} / \mathrm{ml}$ each, $1 \mathrm{mM} \mathrm{Na} 3 \mathrm{VO} 4$, and $1 \mathrm{mM} \mathrm{NaF}$ for $15 \mathrm{~min}$ at $4^{\circ} \mathrm{C}$. Immunoprecipitated proteins were separated by SDS-PAGE, transferred to nitrocellulose membrane and western analysis done with villin monoclonal antibodies.

3.3.12 Separation of Triton-soluble and -insoluble pool. For fractionation of Triton X-100 -soluble and -insoluble pool, MDCK Tet-Off cells expressing VIL/FL, VIL/ACFM or VIL/AYFM were lysed in buffer containing 1\% Triton X-100, $20 \mathrm{mM}$ HEPES, pH 7.2, $150 \mathrm{mM} \mathrm{NaCl}, 2 \mathrm{mM}$ EDTA, $1 \mathrm{mM}$ sodium othovanadate, 50 $\mathrm{mM} \mathrm{NaF}$ and a cocktail of protease inhibitors for 15 min at $4^{\circ} \mathrm{C}$. After centrifugation, supernatants were collected and represented the Triton-soluble pool. The pellets were resuspended in buffer containing 15 mM HEPES, pH 7.5, $150 \mathrm{mM} \mathrm{NaCl}, 1 \%$ Triton $\mathrm{X}-100,1 \%$ sodium deoxycholate, $0.1 \%$ SDS, $10 \mathrm{mM}$ EDTA, $1 \mathrm{mM}$ dithiothreitol, $1 \mathrm{mM}$ sodium orthovanadate and a cocktail of 
protease inhibitors. Samples were vortexed well and incubated on ice for $20 \mathrm{~min}$ on ice. The pellet fraction was centrifuged and the resulting supernatant represented the Triton-insoluble pool. Triton-soluble and -insoluble proteins were separated by SDS-PAGE, transferred to nitrocellulose membrane and western analysis done with villin monoclonal antibodies.

\subsubsection{Identification of apoptotic cells using HOECHST 33258 staining.} MDCK Tet-Off cells transiently transfected with SEYFP-tagged S1-S3 villin truncation were incubated with HOECHST $3325816 \mathrm{~h}$ post-transfection at a concentration of $20 \mu \mathrm{g} / \mathrm{ml}$ for $10 \mathrm{~min}$ at room temperature. Apoptotic cells were distinguished from viable cells by nuclear condensation and DNA fragmentation seen as bright blue fluorescence in the nuclei. Morphological changes corresponding to non-viable cells were determined using an inverted Nikon fluorescence microscope with a CoolSnap FX charge coupled device camera.

\subsection{Results}

3.4.1 The carboxy-terminus of villin is tyrosine phosphorylated. We have previously identified four major tyrosine phosphorylation sites (Y-46, -60, -81 and -256) in the amino terminus of villin (Zhai et al., 2002). Substitution of all four of these sites with phenylalanine allowed us to determine that there were additional phosphorylation sites in the carboxy terminus of villin that were tyrosine phosphorylated in vitro (figure 3.1A). Likewise, expression of this amino-terminal phosphorylation site mutant of villin (VIL/ANFM) in MDCK Tet-Off cells allowed 
Figure 3.1 Tyrosine phosphorylation of wild-type and truncation mutants of villin. (A) Carboxy terminus of villin contains additional tyrosine phosphorylation sites. Four of the tyrosine phosphorylation sites identified in previous studies $(\mathrm{Y}-46$, $-60,-81$ and -256 ) were mutated to phenylalanine (ANFM) (Zhai et al., 2002). The mutant villin protein cloned in the pGEX-4T1 was expressed as a nonphosphorylated protein (VIL/WT (ANFM)) by expression in TKX1 cells in the absence of IAA (see Materials and Methods) or as a phosphorylated protein by expression in TKX1 cells in the presence of IAA (VILT/WT (ANFM)). (B) Aminoterminal phosphorylation site mutant of villin is tyrosine phosphorylated in MDCK Tet-Off cells. Cell extracts from MDCK Tet-Off cells stably transfected with fulllength villin (VIL/FL) or amino-terminal phosphorylation site mutant (VIL/ANFM) were immunoprecipitated with phospho-villin antibody (VP-70782) described previously (Tomar et al., 2004) and western analysis done with phospho-tyrosine antibody. (C) Schematic representation of wild-type and amino-terminal truncation mutants of villin that were expressed as Gst-tagged phosphoryable proteins in TKX1 cells. (D) Expression and tyrosine phosphorylation of wild-type (VILT/WT) and truncation mutant (CT-CT4) villin proteins. The left panel is an SDS-PAGE analysis of recombinant, phosphorylated wild-type (VILT/WT) and amino-terminal truncation mutants of villin. The gels were stained with GelCode Blue. The right panel is a western blot of VILT/WT and truncation mutants of villin probed with phosphotyrosine monoclonal antibody (PY-20). Data are representative of four experiments with similar results. 
A.

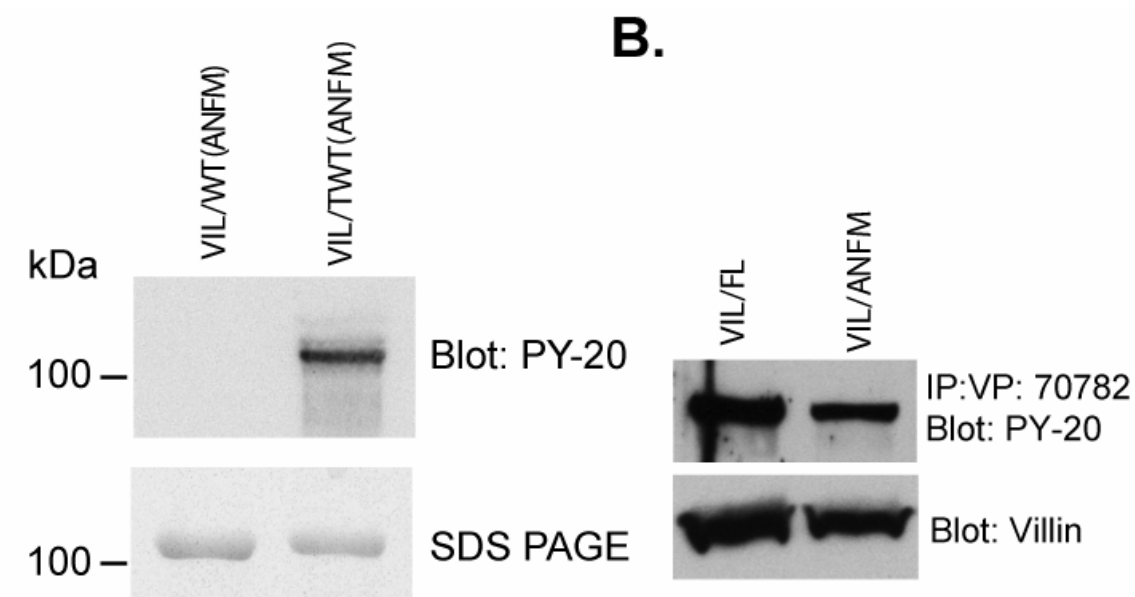

C.
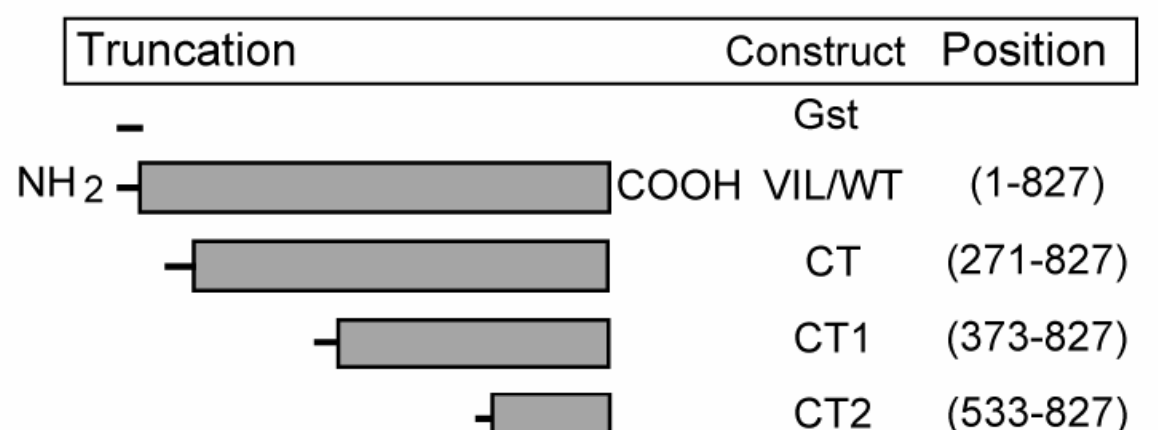
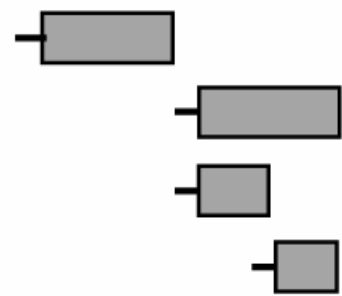

D.

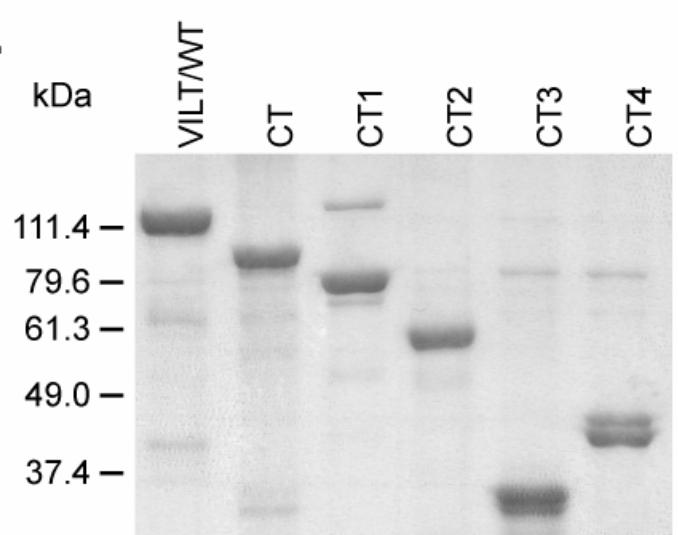

SDS-PAGE
$\mathrm{COOH}$ VIL/WT (1-827)

CT (271-827)

CT1 (373-827)

CT2 (533-827)

CT3 (271-373)

CT4 (373-533)

CT5 (373-437)

CT6 (439-533)

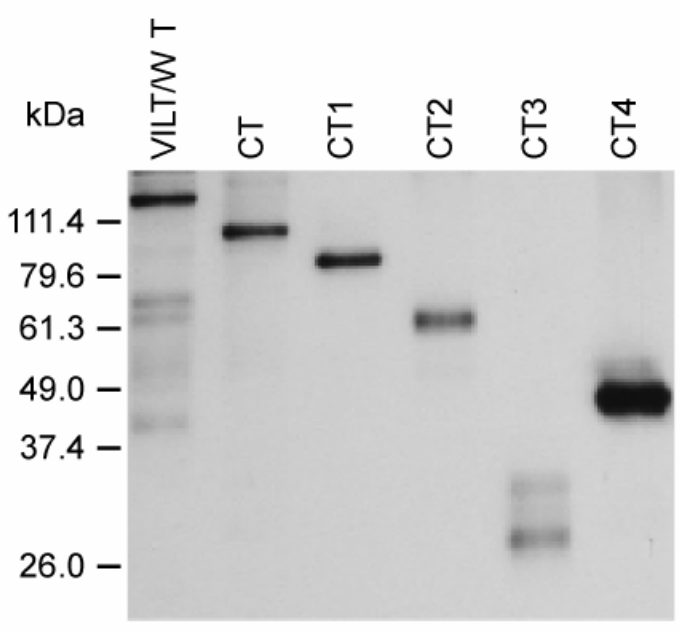

Blot: PY-20 
us to confirm our observation that there were additional phosphorylation sites in the carboxyl terminal domain of villin (figure $3.1 \mathrm{~B}$ ). For these studies, cell extracts of MDCK Tet-Off cells expressing wild-type villin (VIL/FL) or the aminoterminal phosphorylation site mutant of villin (VIL/ANFM) were immunoprecipitated with a phospho-villin antibody (VP-70782) and probed with a phospho-tyrosine antibody (PY-20) essentially as described before (Tomar et al., 2004). Together these studies demonstrate that both in vitro as well as in MDCK cells, the carboxyl terminal domain of villin contribute to the tyrosine phosphorylation of villin. In addition, the identification of at least five tyrosine phosphorylation sites in human plasma gelsolin including a major phosphorylation site in domain S4, led us to speculate that villin may, likewise, have tyrosine phosphorylated residues in the carboxyl-terminal domain(s) (De Corte et al., 1999). To identify these sites in villin as well as to determine the functional significance of these sites, truncation mutants of villin were constructed as shown in figure 3.1C. The constructs consisted of amino-terminal deletions of human villin cDNA engineered into the prokaryotic expression vector pGEX-4T1. Five truncation mutants (CT-CT4) were generated as Gst-tagged fusion proteins in TKX1 cells. The expression of full-length villin and the truncation mutants of villin were assessed by SDS-PAGE and staining with GelCode Blue (figure 3.1D). The apparent molecular masses were in agreement with those predicted for each deletion mutant. To assess tyrosine phosphorylation of these mutants, the proteins were analyzed by western analysis using phosphotyrosine antibodies (PY-20). All four truncation mutants were tyrosine phosphorylated 
(figure 3.1D). These data suggested to us that each of the truncation mutants contained one or more tyrosine phosphorylated residues. To identify these phosphorylation sites, smaller truncation mutants were generated (CT5 and CT6, figure $3.1 \mathrm{C})$. Individual tyrosine residues in these shorter truncation fragments (CT2, СT3, СT5 and CT6) were substituted with phenylalanine and the effect of these mutations on the levels of phosphorylation was determined (Zhai et al., 2002).

\subsubsection{Identification of one major and two minor phosphorylation sites in}

CT2. The truncation mutant of villin $\mathrm{CT} 2$ contains four tyrosine residues $(\mathrm{Y}-555$, $-604,-681$ and -725 ) between amino acids $533-827$ of human villin that could be phosphorylated. We used site-directed mutagenesis to substitute each of these tyrosine residues with phenylalanine in the truncation mutant CT2. The mutants were expressed in TKX1 cells, and the phosphorylation status of the mutant proteins was analyzed by western analysis using an anti-phosphotyrosine antibody (figure 3.2A). Densitometric analysis was done by determining the ratio of phosphorylated protein to total protein for each sample. Mutation of Y725 to F significantly decreased the level of tyrosine phosphoryaltion in the truncation mutant CT2 $(54.3 \pm 3.3 \%, n=4, p<0.001)$. In contrast mutation of $\mathrm{Y} 555$ or Y604 to $\mathrm{F}$ decreased the level of tyrosine phosphorylation of the villin construct CT2 to a lesser extent $(25.0 \pm 5.7 \%$ and $37.5 \pm 4.1 \%$ respectively, $n=4, p<$ 0.001). This suggested that CT2 contains one major (Y725) and two minor (Y555 and Y604) tyrosine phosphorylated residues. To confirm this observation, double mutants of these sites were generated in CT2 (figure 3.2B). 
Figure 3.2 Identification of one major and two minor tyrosine phosphorylation sites in villin truncation mutant CT2. (A) Mutant villin proteins were generated by substitution of tyrosine residues at $555,604,681$ or 725 with phenylalanine in villin truncation mutant CT2 [CT2(Y555F); CT2(Y604F); CT2(Y681F); CT2(Y725F)]. The villin mutants were expressed as Gst-tagged phosphoryable proteins in TKX1 cells. The samples were normalized for protein content and subjected to SDS-PAGE and western analysis using phosphotyrosine antibody (PY-20). (B) A villin mutant was generated by substituting two of the three identified tyrosine phosphorylation sites in different combinations (CT2 (Y604F, Y555F); CT2 (Y725F, Y604F); CT2 (Y725F, Y555F). Villin truncation mutant $\mathrm{CT} 2$ as well as these phosphorylation site mutants were purified from TKX1 cells, separated by SDS-PAGE and stained with GelCode Blue. western analysis was done with a phosphotyrosine antibody. Tyrosine phosphorylation of CT2 and phosphorylation site mutants of CT2 was quantified using Scion Image software. The values for phosphorylation site mutants of CT2 were normalized to the phosphorylation of truncation mutant CT2 (which was set as 100). Statistically significant values (compared with CT2) are marked with an asterisk $(p<0.001)$. 
A.

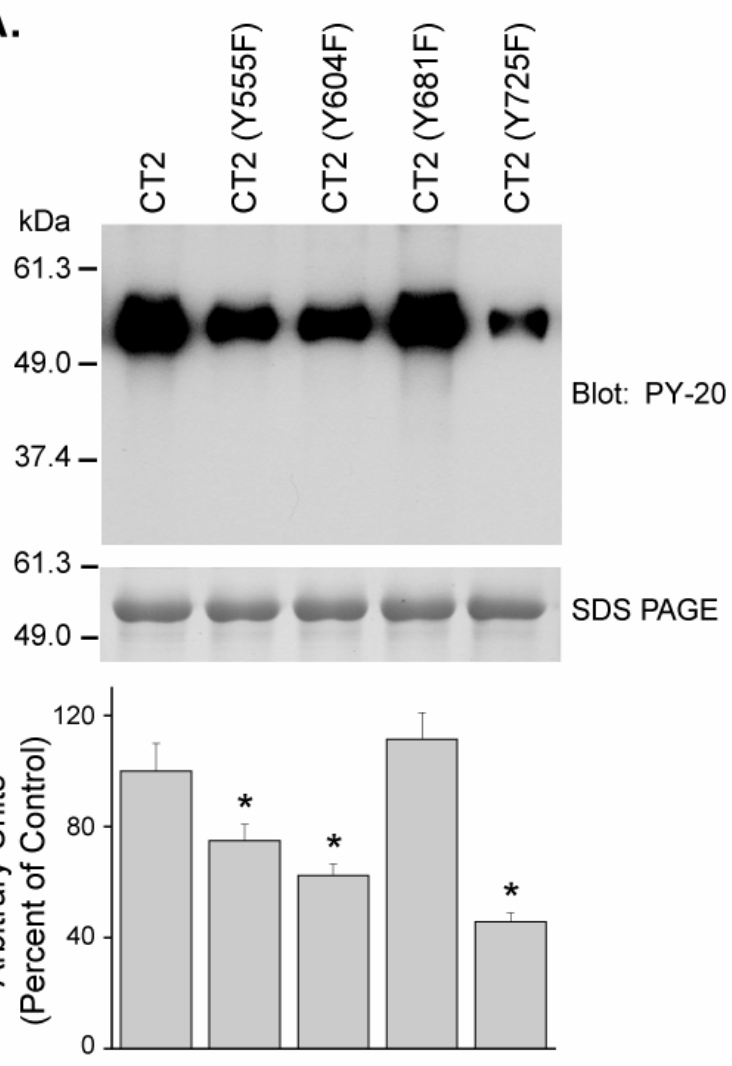

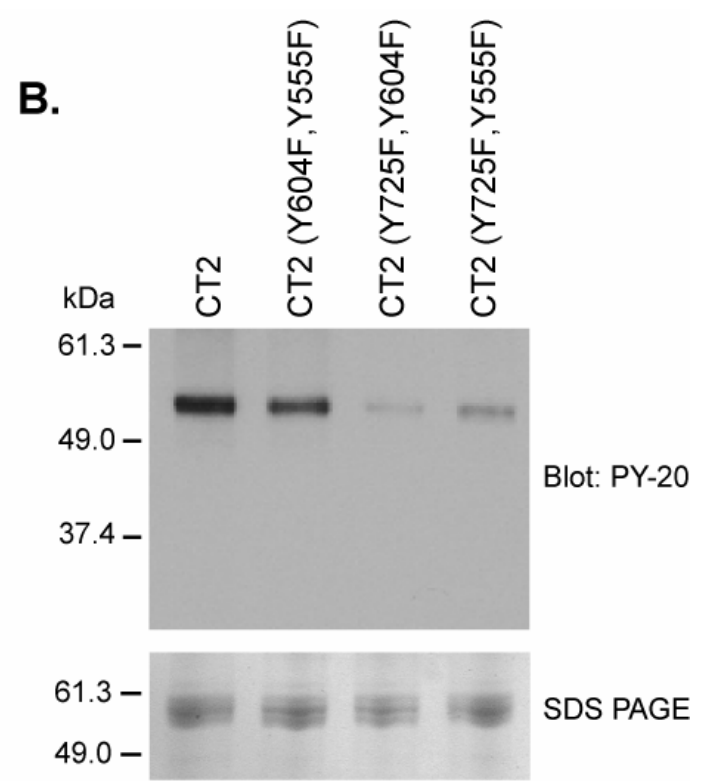

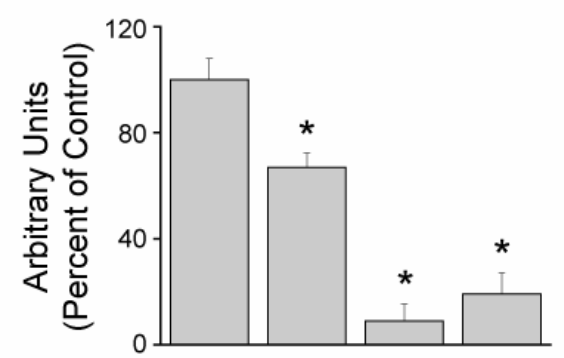


Western analysis of the double mutants using phosphotyrosine antibodies indicated essentially a complete loss of tyrosine phosphorylation with double mutants $Y 725,604 F(91 \%, n=4, p<0.001)$ and $Y 725,555 F(81 \%, n=4, p<$ 0.001). In contrast, the double mutant $\mathrm{Y} 604,555 \mathrm{~F}$ resulted in a relatively smaller decrease in the total phosphorylation level in the truncation mutant CT2 $(33.0 \pm 5.3 \%, n=4, p<0.001)$. These results indicate the presence of one major and two minor sites in human villin protein between a.a. 533 and 827 .

\subsubsection{Identification of two major phosphorylation sites in villin truncation}

mutant CT3. Villin truncation mutant CT3 contains three putative phosphoryable tyrosine residues, Y286, Y296 and Y324. We mutated each of these tyrosine residues to a phenylalanine and determined the effect of the mutation on the levels of phosphorylation in the truncation mutant CT3. Equal amounts of the proteins were separated by SDS-PAGE and western analysis done with phosphotyrosine antibodies (PY-20). As shown in figure 3.3, mutation of Y286 as well as Y324 significantly decreased the levels of tyrosine phosphorylation in the truncation mutant CT3 $(99.0 \pm 1.0 \%$ and $65.9 \pm 3.7 \%$ respectively, $n=4, p<$ 0.001). On the other hand, mutation of Y296 to phenylalanine had no effect on the phosphorylation levels of the truncation mutant CT3. These data demonstrate that in addition to $\mathrm{Y} 555, \mathrm{Y} 604$ and $\mathrm{Y} 725$, the carboxyl-terminal domain of villin contains two additional tyrosine residues that can be phosphorylated in vitro, namely Y286 and Y324. 


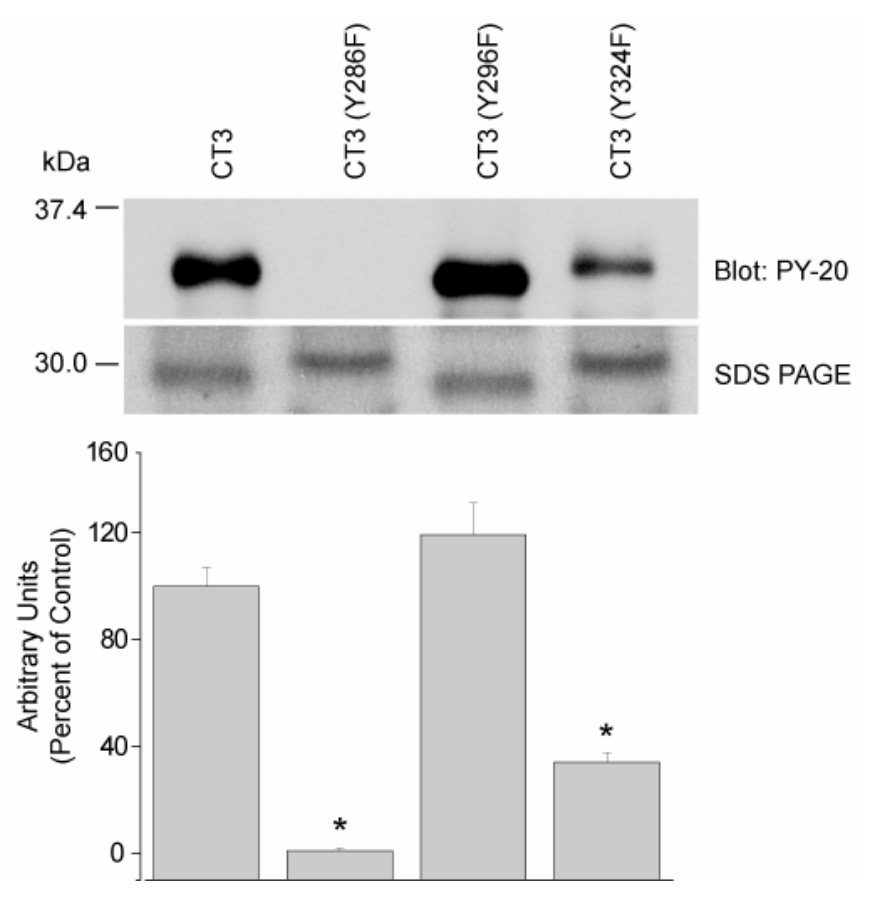

Figure 3.3 Tyrosine 286 and 324 are the major phosphorylation sites in the villin truncation mutant CT3. Mutant villin proteins were generated by substitution of tyrosine residues at 286,296 or 324 with phenylalanine in villin truncation mutant CT3 [CT3 (Y286F); CT3 (Y296F); CT3 (Y324F)]. The villin mutants were expressed as Gst-fused phosphoryable proteins in TKX1 cells. The samples were normalized for protein content and subjected to SDS-PAGE and western analysis using phosphotyrosine antibody (PY-20). Tyrosine phosphorylation of CT3 and phosphorylation site mutants of CT3 was quantified using Scion Image software. The values for phosphorylation site mutants of CT3 were normalized to the phosphorylation of truncation mutant CT3 (which was set as 100). Statistically significant values (compared with CT3) are marked with an asterisk $(p<0.001)$. 


\subsubsection{Y461 is the major phosphorylation site in the villin truncation mutant}

CT4. As shown in figure 3.1D, the villin truncation CT4 is also tyrosine phosphorylated suggesting that this truncation contains one or more tyrosine residues that can be phosphorylated in vitro. Human villin contains 7 tyrosine residues within this domain that could be phosphorylated in vitro. To determine these sites of phosphorylation, we generated two additional truncation mutants, CT5 and CT6, which encompass the amino acid residues between 373 and 533 (similar to CT4, figure 3.1 C). CT5 contains four tyrosine residues namely Y-422, $-427,-431$ and -433 . Mutation of either of these sites to phenylalanine resulted in less than $5 \%$ decrease in the phosphorylation levels suggesting that these are minor sites of phosphorylation, if at all, in the villin truncation CT4 (data not shown). The truncation mutant CT6 contains three tyrosine residues namely, $Y$ $-461,-444$ and -470 and mutation of one of these residues, Y461, completely $(99 \%, \mathrm{n}=5, \mathrm{p}<0.001)$ abolished the phosphorylation in the truncation CT6 (figure 3.4A). To confirm that $\mathrm{Y} 461$ was the only major phosphorylation site in CT4, we also generated a point mutant, Y461F, in CT4 (figure 3.4B). The expression and analysis of this mutant demonstrated that $Y 461$ was the single key phosphorylation site in CT4 (a.a. 373-534 of human villin). In conclusion, in this study we have identified six tyrosine phosphorylation sites in the carboxylterminal domain of villin between a.a. 271-827 of human villin. All of these sites are well conserved in villin across different species. Three of these sites are also conserved among other proteins of the villin family including gelsolin, adseverin 
Figure 3.4 Identification of tyrosine residue 461 as the major phosphorylated residue in villin truncation mutant $\mathrm{CT} 4$. (A) Mutant villin proteins were generated by substitution of tyrosine residues at 444,461 or 470 with phenylalanine in villin truncation mutant CT6 [CT6 (Y444F); CT6 (Y461F); CT6 (Y470F)]. (B) Tyrosine Y461 in villin truncation mutant CT4 was substituted with phenylalanine (CT4 (Y461F). The values for phosphorylation site mutants were normalized to the phosphorylation of truncation mutant CT6 or CT4 (which was set as 100). Statistically significant values are marked with an asterisk $(p<0.001)$. (C) Sequence alignment of villin and related proteins of the villin superfamily. The top panel shows alignment of villin sequences from human, mouse and chicken. The bottom panel shows alignment of homologous sequences in gelsolin, adseverin and CapG. (D) Substitution of all ten tyrosine residues at positions 46, $60,81,256,286,324,461,555,604$ and 725 with phenylalanine (VILT/WT (AYFM)) eliminates tyrosine phosphorylation in full-length recombinant villin protein. Tyrosine phosphorylation of full-length recombinant villin protein (VILT/WT) was compared with villin mutant VILT/WT(AYFM) using a phosphotyrosine antibody or a phospho-villin antibody (VP-70782) described previously (Tomar et al., 2004). (E) Mutation of all ten identified tyrosine residues abolishes tyrosine phosphorylation of villin in MDCK Tet-Off cells. Cell extracts from MDCK Tet-Off cells stably transfected with VIL/FL or VIL/AYFM were immunoprecipitated with phospho-villin antibody (VP-70782) and western analysis done with phospho-tyrosine antibody. 
A.
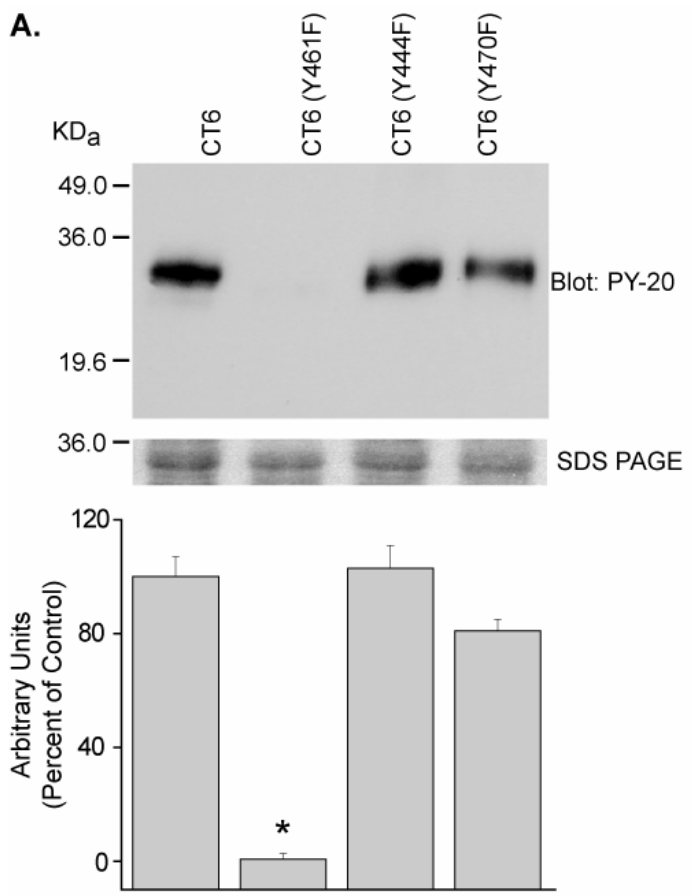

B.
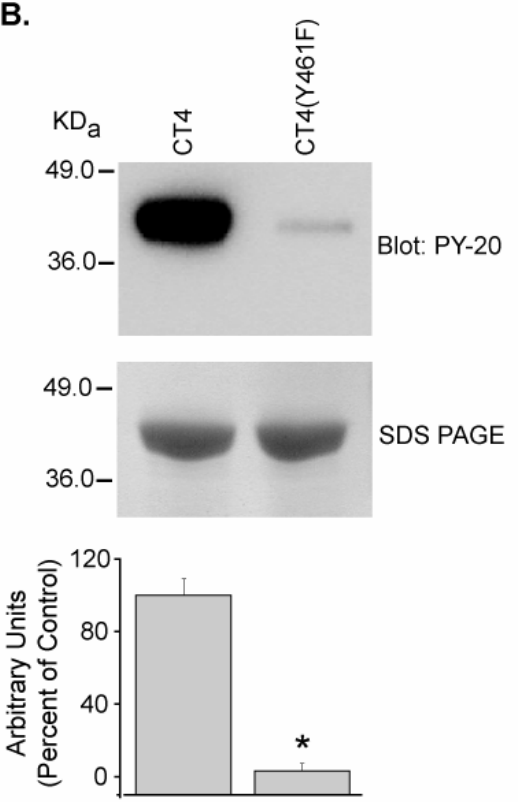

c.

\begin{tabular}{|c|c|c|c|c|c|c|}
\hline & Y286 & Y324 & Y461 & Y555 & Y604 & Y725 \\
\hline$\underset{\text { Human }}{\text { Villin }}$ & HEDCYILDQ & KAKQYPPST & TASAYQAVI & QSCCYLWCG & GKAPYANTK & NTKSYED \\
\hline Mouse & HEDCYILDQ & KAKQYPPST & AASAYQAVL & PSCCYLWCG & GKAPYANTK & NTKSYDD \\
\hline Chicken & HEDCYILDQ & KAKNYLAST & AASAYQAVF & PSSCYLWYG & GKTSYANSK & DRKSYDE \\
\hline Gelsolin & & KAKQYPPST & TASAYQAVI & QSCCYLWCG & GKAPYANTK & \\
\hline Adseverin & & TKMDYPKQT & AASAYQAVL & PSCCYLWCG & GKAPYANTK & \\
\hline CAPG & & QQMNYAPNT & AASAYQAVF & PSSCYLWYG & GKTSYANSK & \\
\hline
\end{tabular}

D.

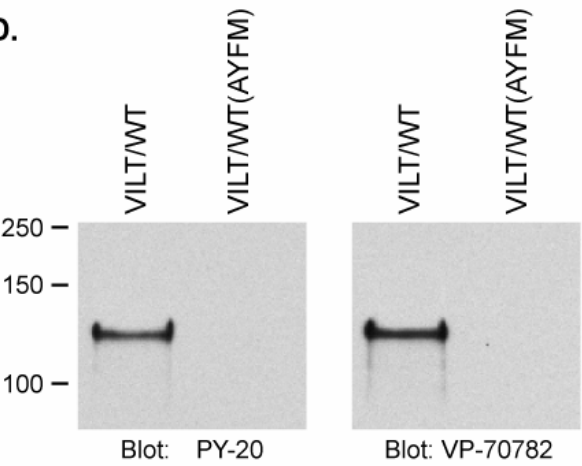

E.

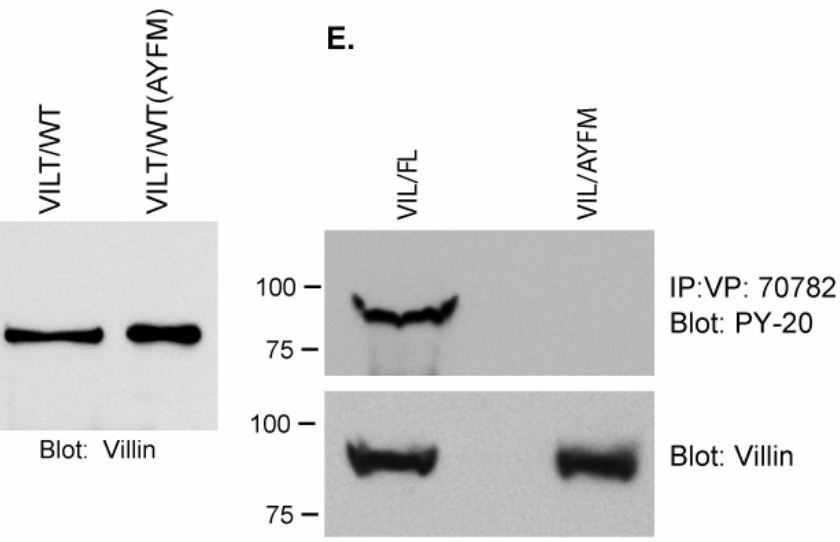


and CapG (figure 3.4C). Two of these sites Y324 and Y604 in human villin have been identified as minor sites that are phosphorylated in human plasma gelsolin (Y576 and Y624 respectively in gelsolin) (De Corte et al., 1999). Mutation of all ten sites (VILT/WT(AYFM)) in villin, four previously identified (Y-46, -60, -81 and $-256)$ as well as the six sites identified in this study (Y-286, -324, $-461,-555,-604$ and -725 ) resulted in the complete loss of tyrosine phosphorylation in full-length recombinant villin protein (figure 3.4D). These data were confirmed in MDCK TetOff cells stably transfected with this villin mutant lacking all ten identified tyrosine phosphorylation sites (VIL/AYFM) (figure 3.4E). Collectively with our previous study we have now identified ten tyrosine residues in human villin that are phosphorylated both in recombinant villin protein as well as in MDCK cells.

We also examined the stoichiometry of tyrosine phosphorylated villin in MDCK cells expressing full-length villin (VIL/FL), villin mutant lacking the four amino-terminal phosphorylation sites previously identified (VIL/ANFM), villin mutant lacking the six carboxyl-terminal phosphorylation sites identified in this study (VIL/ACFM) and a villin mutant lacking all ten identified tyrosine phosphorylation sites (VIL/AYFM). MDCK cells stably expressing wild-type and mutant villin proteins were lysed and immunoprecipitated using PY-20. Since the commercially available villin monoclonal antibody cannot be used for immunoprecipitation and since the phospho-villin antibody VP-70782 binds to selective tyrosine phosphorylated epitopes of villin, we reasoned that a phosphotyrosine antibody would bind phosphorylated villin proteins with comparable affinity. We have previously demonstrated that PY-20 immunoprecipitates 
tyrosine phosphorylated villin protein (Khurana et al., 1997; Tomar et al., 2004). Quantitative immunoprecipitation studies demonstrated that in MDCK cells VIL/FL, VIL/ANFM and VIL/ACFM were tyrosine phosphorylated while villin mutant VIL/AYFM was not (figure 3.5). Densitometric analysis revealed that mutation of the amino-terminal phosphorylation sites or the carboxyl-terminal phosphorylation sites resulted in a $30.6 \pm 12.22$ and $56.79 \pm 10.96$ percent decrease respectively in the phosphorylation levels of mutant villin proteins compared to wild-type villin protein. Mutation of all ten tyrosine phosphorylation sites (VIL/AYFM) resulted in the complete loss of phosphorylation of the villin protein in MDCK cells (98.52 \pm 0.47 percent decreased compared to VIL/FL). These studies demonstrate that the ten tyrosine phosphorylation sites identified in recombinant villin protein are also tyrosine phosphorylated in MDCK cells with a comparable stoichiometry.

\subsubsection{Functional significance of tyrosine phosphorylation sites identified in} the carboxy-terminus of villin. Each of the six tyrosine phosphorylation sites identified in this study were mutated individually in full-length human villin cDNA to phenylalanine (VILT/WT(Y286F); VILT/WT(Y324F); VILT/WT(Y461F); VILT/WT(Y555F); VILT/WT(Y604F); VILT/WT(Y725F)), expressed in TKX1 cells and purified as Gst-tagged tyrosine phosphorylated proteins. All six mutants were tyrosine phosphorylated but lacked one of the identified tyrosine residues (figure 3.6A). In order to assess whether the single point mutants maintained the conformation of the wild-type villin protein, the unfolding profiles of wild-type 


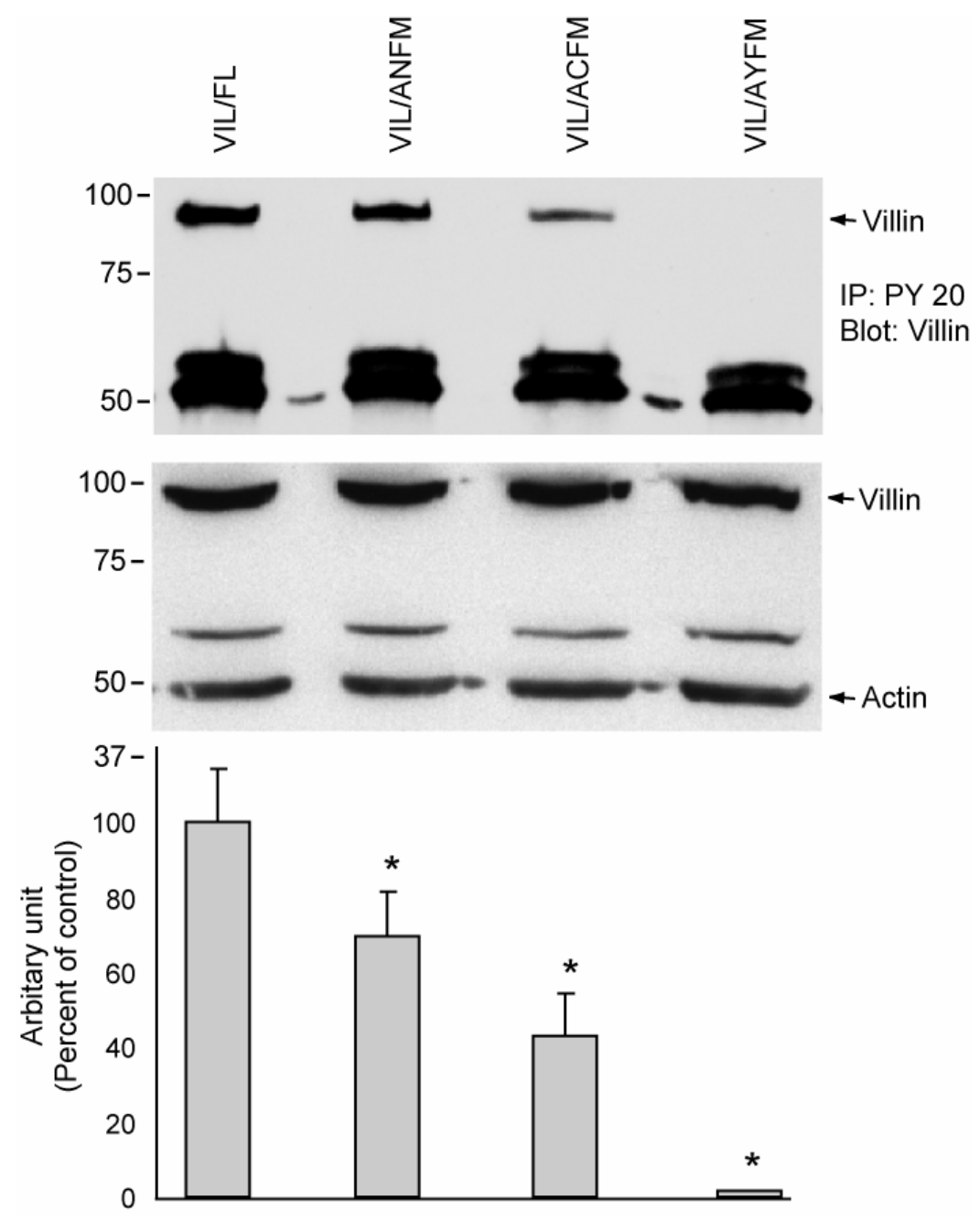

Figure 3.5 Tyrosine phosphorylation of wild-type and mutant villin proteins. Cell extracts from VIL/FL, VIL/ANFM, VIL/ACFM and VIL/AYFM were immunoprecipitated with phospho-villin antibody (VP-70782) and western analysis done with phospho-tyrosine antibody PY-20 (upper panel). Middle panel is a western blot with anti-villin monoclonal antibody and the bottom panel is a western blot with anti-actin antibody. 
Figure 3.6 Regulation of actin dynamics by the carboxyl-terminal tyrosine phosphorylation sites in villin. (A) Expression of full-length villin proteins with point mutations at position $286,324,461,555,604$ or 725 . Tyrosine residues at these positions were substituted with phenylalanine in full-length villin and expressed as recombinant proteins in TKX1 cells. (B) Urea-induced, equilibriumunfolding transition of wild-type and mutant villin proteins. Urea-induced denaturation of wild-type and mutant villin proteins was measured by the change in tryptophan fluorescence maxima at an excitation wavelength of $280 \mathrm{~nm}$ and an emission scan from 300 to $420 \mathrm{~nm}$. (C) Effect of wild-type and phosphorylation site mutant villin proteins on the kinetics of actin polymerization. Pyrene-labeled G-actin was incubated with VIL/WT, VILT/WT or mutant mutant villin proteins in polymerization-inducing buffer, and fluorescence intensity was measured over time. (D) Effect of wild-type and phosphorylation site mutant villin proteins on the kinetics of actin depolymerization. Pyrene-labeled F-actin in the presence of VIL/WT, VILT/WT or mutant villin proteins was diluted in actin depolymerizing buffer, and the decrease in fluorescence intensity was followed over time. (E) Effect of wild-type and phosphorylation site mutant villin proteins on the kinetics of actin-capping. Polymerization of G-actin at barbed ends was nucleated by Factin (290 nM) seeds in the absence (control) or presence of non-phosphorylated (VIL/WT), phosphorylated (VILT/WT) or mutant villin proteins. 
A.

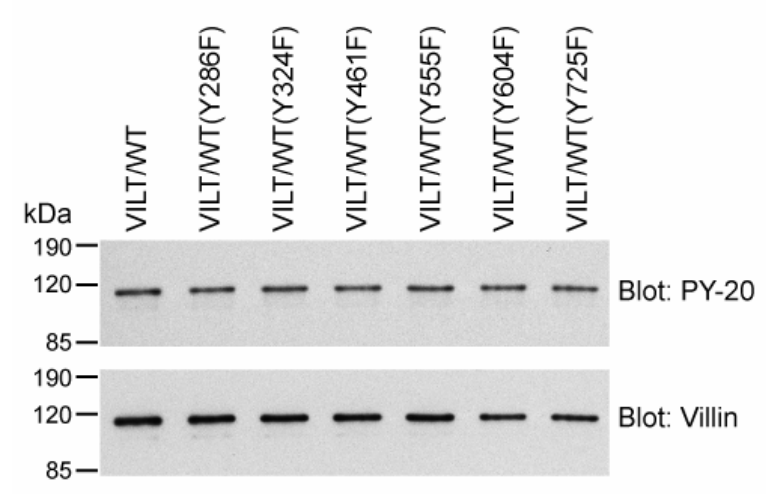

C.

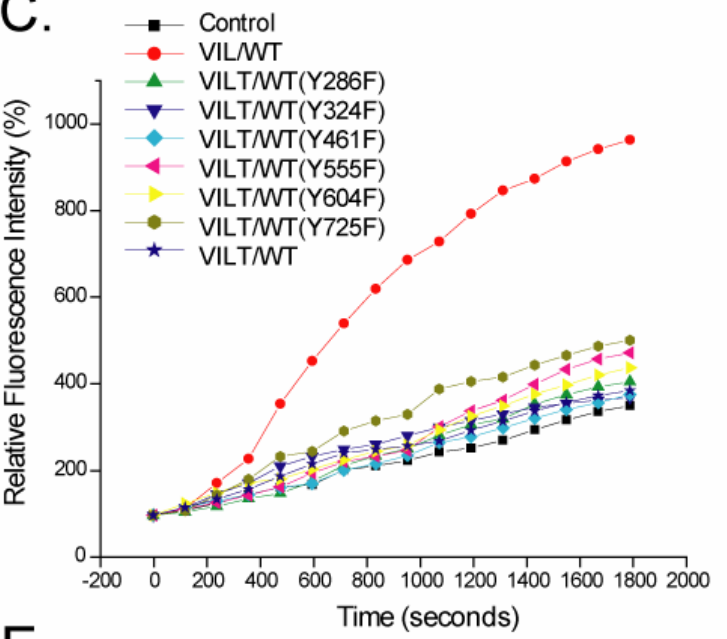

E

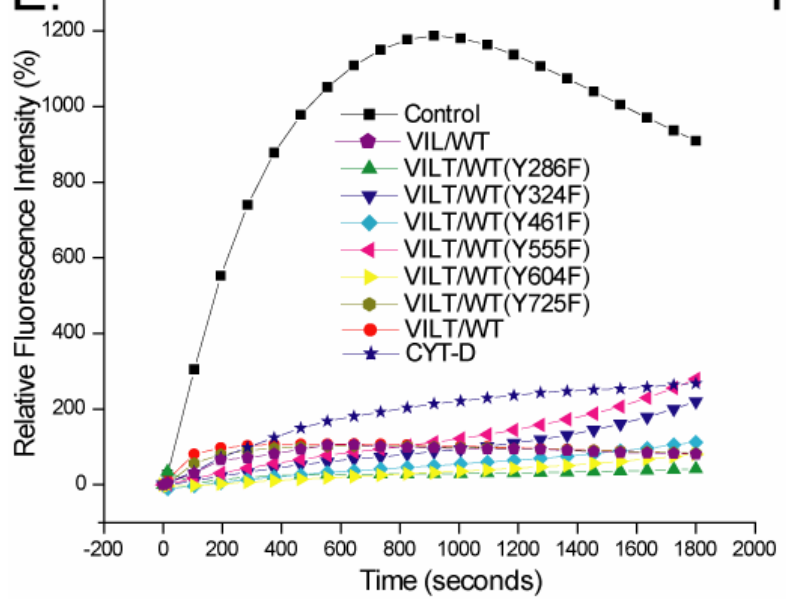

B.

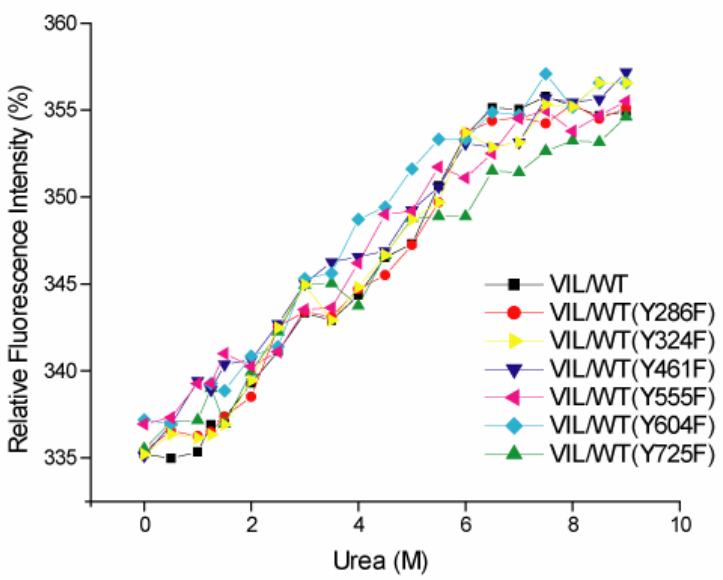

D.

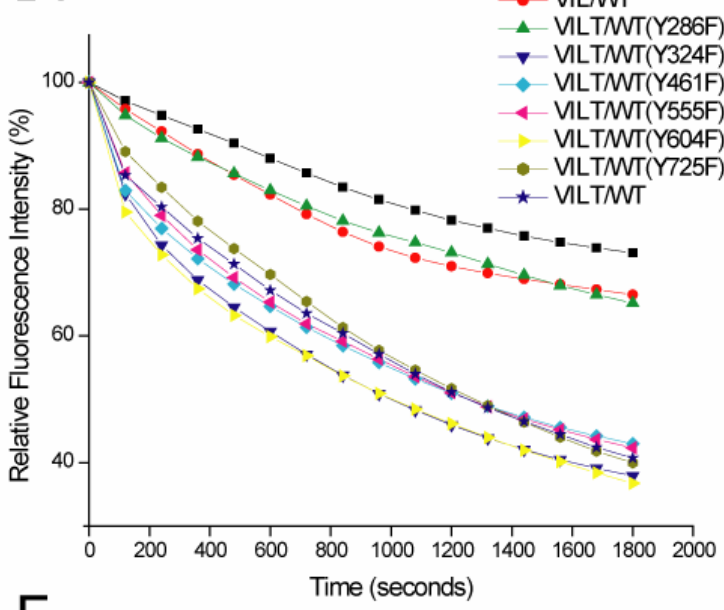

F.

\begin{tabular}{c|c|c|c}
\multicolumn{2}{l|}{$\begin{array}{l}\text { Phosphorylation } \\
\text { sites }\end{array}$} & \multicolumn{3}{|c}{ F-actin } \\
\cline { 2 - 4 } *Nucleation & Severing & Capping \\
\hline & & & \\
Y286 & + & + & - \\
Y324 & + & - & - \\
Y461 & + & - & - \\
Y555 & + & - & - \\
Y604 & + & - & - \\
Y725 & + & - & - \\
\hline
\end{tabular}


and mutant villin proteins expressed in the absence of IAA (non-phosphorylated proteins) as a function of the urea concentration were recorded by measuring the intrinsic tryptophan fluorescence emission spectrum essentially as described before (Kumar et al., 2004b). The results indicate that single point mutants express similar unfolding transitions as wild-type villin, thus maintaining a comparable overall conformation as the wild-type recombinant villin protein (figure 3.6B). Full-length non-phosphorylated villin (VIL/WT), phosphorylated villin (VILT/WT) and the phenylalanine substituted single point mutants were used in a pyrene based actin-polymerization and -depolymerization assay as described before (Zhai et al., 2002). As shown in figure 3.6C, the addition of non-phosphorylated full-length villin (VIL/WT) increased the initial rate of actin polymerization compared to the polymerization kinetics of actin alone (control). In contrast, tyrosine phosphorylated full-length villin (VILT/WT) resulted in a lag phase and decreased the rate of actin polymerization. This is consistent with our previously published observation that tyrosine phosphorylation inhibits the actinnucleating function of villin (Zhai et al., 2001; Zhai et al., 2002; Kumar and Khurana, 2004). Mutation of any of the six identified phosphorylation sites individually from $Y$ to $F$, did not rescue the actin-nucleating property of nonphosphorylated full-length villin protein. These data demonstrate that phosphorylation at any five of the six identified tyrosine residues inhibits actin nucleation by villin. Thus, like the amino-terminal phosphorylation sites, the carboxyl-terminal phosphorylation sites also inhibit the actin-nucleating activity of villin (Zhai et al., 2002). These studies suggest that the entire villin core and 
multiple tyrosine phosphorylation sites in the villin core could regulate actin nucleation by villin.

Next we examined the effect of tyrosine phosphorylation of villin on F-actin depolymerization. Non-phosphorylated full-length villin protein (VIL/WT) increased the depolymerization of F-actin compared with control samples (figure 3.6D). Furthermore, phosphorylation of villin enhanced its actin-depolymerizing property, similar to our previous reports (Zhai et al., 2001; Zhai et al., 2002; Kumar and Khurana, 2004). Mutation of $Y 286$ to $F$ resulted in actin severing by villin comparable to that of non-phosphorylated full-length villin protein, while all other mutants (Y324F, Y461F, Y555F, Y604F or Y725F) behaved like VILT/WT. These data suggest that Y286 is the major phosphorylation site that regulates the actin-depolymerizing function of villin. Taken together with our previous study, we have now determined three tyrosine residues, namely $Y 46, Y 60$ and $Y 286$, in the amino-terminal domain (S1-S3) of villin that regulate its actin-depolymerizing activity (Zhai et al., 2002).

The ability of VIL/WT, VILT/WT and the phosphorylation site mutants of villin to bind to the plus or barbed end of actin filaments was tested by following the polymerization kinetics of pyrene-labeled G-actin from barbed ends under polymerization conditions where there is little or no growth from the pointed end. The concentration of calcium $(2.5 \mu \mathrm{M})$ used in this assay has been shown to be saturating for capping but not severing of actin filaments by villin (Northrop et al., 1986b). As shown in figure 3.6E, addition of pyrene-labeled G-actin in the presence of F-actin seeds results in significant and rapid actin polymerization 
over time. VIL/WT shows decreased rates of polymerization in the presence of $2.5 \mu \mathrm{M}$ calcium, consistent with capped barbed ends. VILT/WT as well as the phosphorylation site mutants of villin likewise capped the barbed ends of actin. The inhibition of F-actin polymerization in the presence of $20 \mathrm{nM}$ Cytochalasin D confirms the fact that capping of the barbed end is indeed the cause of abolished actin polymerization. These data show that phosphorylated villin caps actin filaments very similar to non-phosphorylated villin protein. Further these results demonstrate that the actin-capping activity of villin is not regulated by tyrosine phosphorylation.

\subsubsection{Regulation of villin-induced cell migration by carboxyl-terminal} phosphorylation site mutants. Because we have previously shown that tyrosine phosphorylation of villin regulates cell migration, we examined the ability of the carboxyl-terminal phosphorylation site mutants of villin to regulate this function of villin (Tomar et al., 2004). For these studies, HeLa Tet-Off cells were stably transfected with wild-type or the individual phosphorylation site mutants of villin. Clones expressing comparable levels of wild-type and phosphorylation site mutant proteins were selected for these studies (figure 3.7). As shown in supplementary figure $3.7 \mathrm{~B}$ b1-b3 and as reported previously, overexpression of wild-type villin results in loss of stress fibers and redistribution of F-actin at or near the cell surface compared with untransfected cells which appear more flat and exhibit stress fibers (figure 3.7B ${ }_{\mathrm{a} 1-\mathrm{a} 3}$ ) (Tomar et al., 2004). Mutation of any of the six carboxyl-terminal phosphorylation sites individually did not result in any significant changes in either the distribution of villin or F-actin (figure 3.7B c-h). 
Figure 3.7 Phosphorylation of villin at Y286, Y324, Y461, Y555, Y604 or Y725 is not required for villin-induced changes in actin reorganization or cell shape change. (A) HeLa Tet-Off cells were stably transfected with wild-type (VIL/FL) and phosphorylation site point mutants of villin, namely VIL/Y286F; VIL/Y324F; VIL/Y461F; VIL/Y555; VIL/Y604F; VIL/Y725F; or amino-terminal phosphorylation site mutant (VIL/ANFM) or the carboxyl-terminal phosphorylation site mutant (VIL/ACFM). This figure shows representative clones of each villin construct transfected in HeLa Tet-Off cells. (B) Villin expression results in reorganization of the actin cytoskeleton. HeLa Tet-Off cells transfected with wild-type and mutant villin proteins were analyzed by confocal microscopy. Double staining of villin $\left(a_{1}-j_{1}\right)$ and $F$-actin $\left(a_{2}-j_{2}\right)$ was performed using villin monoclonal antibodies (1:100) and Cy3 conjugated anti-mouse $\lg$ (1:100) and Alexa-Phalloidin 488 (1 $\mu \mathrm{g} / \mathrm{ml}$ ), respectively. Composite images of villin (red) and F-actin staining (green) are shown. Merged images show co-localization of villin and F-actin $\left(a_{3}-j_{3}\right)$. Bars, $10 \mu \mathrm{m}$. (C) HeLa Tet-Off cells expressing equal amounts of wild-type or phosphorylation site point mutants were used in wound-healing experiments. All villin expressing cells (wild-type as well as mutants) migrated significantly faster than villin null cells $(p<0.01, n=24)$. VIL $(-)$ cells refer to each clone cultured in the presence of doxycycline, whereas VIL $(+)$ refers to the same clone cultured in the absence of doxycycline. The error bars are the measured SEM. 

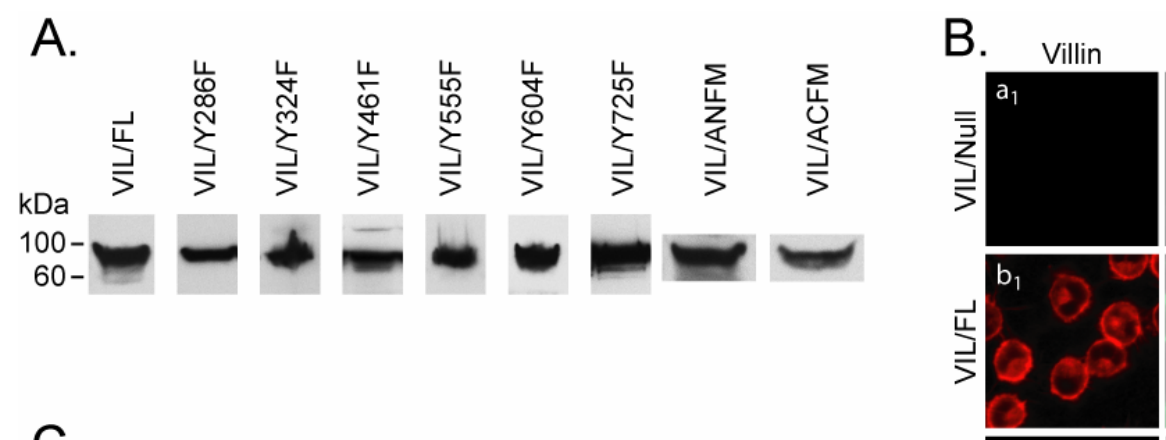

F-Actin

Merge
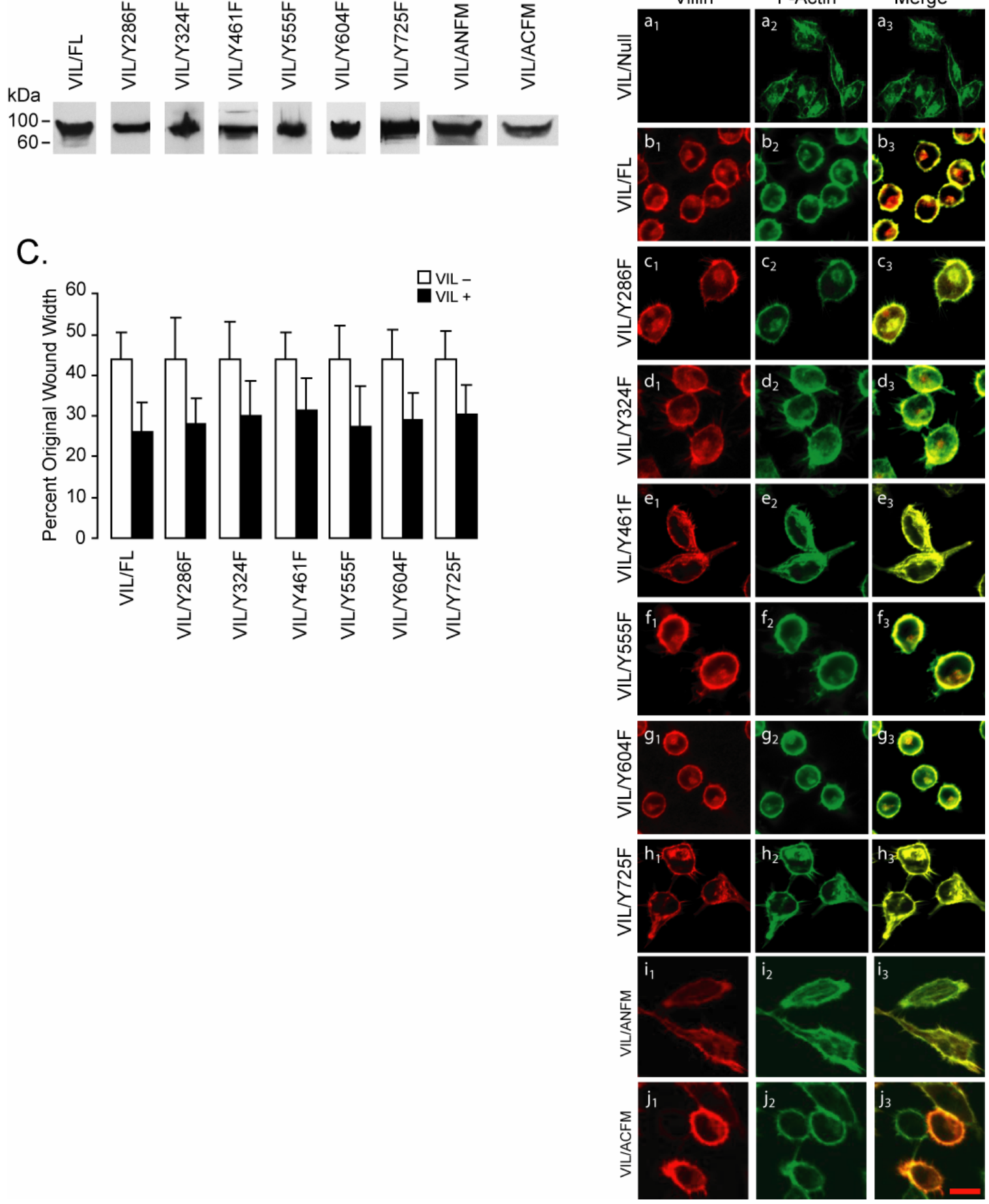
Intracellular distribution of F-actin and villin were also examined in HeLa cells expressing the villin mutants VIL/ACFM as well as VIL/ANFM. Clones expressing comparable levels of VIL/ACFM and VIL/ANFM were selected for these studies (figure 3.7A). There was no significant change in either in the cell morphology or the distribution of villin or F-actin in cells expressing VIL/ACFM compared with VIL/FL (compare figure 3.7B $\mathrm{j1}_{\mathrm{j} 3}$ and figure 3.7B $\mathrm{b} 1-\mathrm{b} 3$ ). In contrast, the villin mutant VIL/ANFM demonstrated a cell shape change consistent with villin-null cells (figure $3.7 \mathrm{~B}_{11-\mathrm{i} 3}$ and figure $3.7 \mathrm{~B}_{\mathrm{a} 1-\mathrm{a} 3}$ ). This is similar to the observation made with individual amino-terminal phosphorylation site mutants (Tomar et al., 2004). Together with our previous studies, these data suggest that changes in the microfilament structure and cell morphology are regulated by the amino-terminal phosphorylation sites (Tomar et al., 2004). Next, we examined the effects of these point mutants on villin-induced cell migration. Consistent with our previous report, HeLa Tet-Off cells overexpressing wild-type $(\mathrm{VIL}+)$ villin migrated faster than villin null cells (VIL -) (Tomar et al., 2004). The carboxylterminal phosphorylation site point mutants behaved like HeLa cells expressing wild-type villin and individually did not regulate villin induced cell migration (figure 3.7C). To test the possibility that these sites may collectively be important to villin's function in cell migration, we examined the villin mutant lacking all six carboxyl-terminal phosphorylation sites (VIL/ACFM). To our surprise, mutation of all six carboxyl-terminal tyrosine residues to phenylalanine significantly inhibited villin-induced increase in cell migration (figure 3.8A). The villin mutant VIL/ACFM migrated significantly slower than VIL/FL and more like the villin-null cells 
Figure 3.8 Collectively the carboxyl-terminal tyrosine residues regulate villininduced cell migration. (A) Villin mutants lacking all four amino-terminal phosphorylation sites (VIL/ANFM) or villin mutant lacking all six carboxyl-terminal phosphorylation sites (VIL/ACFM) were cloned in pTRE- $6 \times$ His and transfected in HeLa Tet-Off cells. HeLa Tet-Off cells expressing equal amounts of VIL/FL, VIL/ANFM, or VIL/ACFM, were used in a wound-healing assay. Cells expressing VIL/WT migrate significantly faster than villin-null (VIL -) cells. VIL/ANFM and VIL/ACFM, migrate like villin-null cells. The error bars are the measured SEM, and the asterisk $\left({ }^{*}\right)$ denotes statistically significant values $(p<0.01, n=24$, compared with VIL/FL). (B) The carboxyl-terminal phosphorylation sites in villin regulate villin induced lamellipodia formation. MDCK Tet-Off cells transfected with SEYFP-tagged full-length (SEYFP/VIL), villin mutant lacking the aminoterminal phosphorylation sties (SEYFP/ANFM) or villin truncation mutant lacking the carboxyl-terminal domain (SEYFP/S1-S3) were treated with EGF $(50 \mathrm{ng} / \mathrm{ml})$ and time-lapse images recorded 5 min after the addition of EGF for a total of 35 min. Bar $10 \mu \mathrm{m}$. (C) Lamellipodial protrusion rates were determined as described in Methods. Average velocity of lamellipod formation is denoted in $\mu \mathrm{m} / \mathrm{min}$. The error bars are the measured SEM, and the asterisk $\left(^{*}\right)$ denotes statistically significant values ( $p<0.001, n=10$, compared with VIL/FL). 


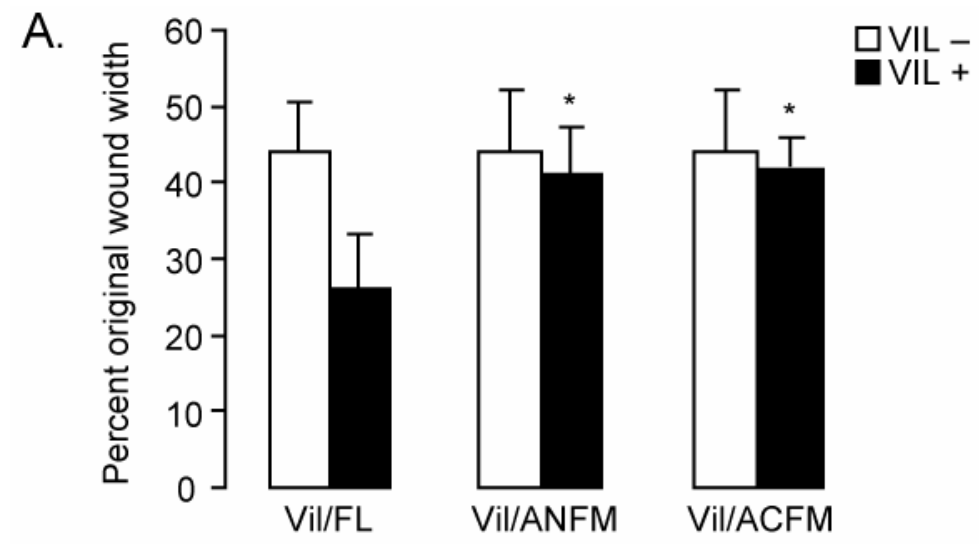

B. $5 \mathrm{~min}$
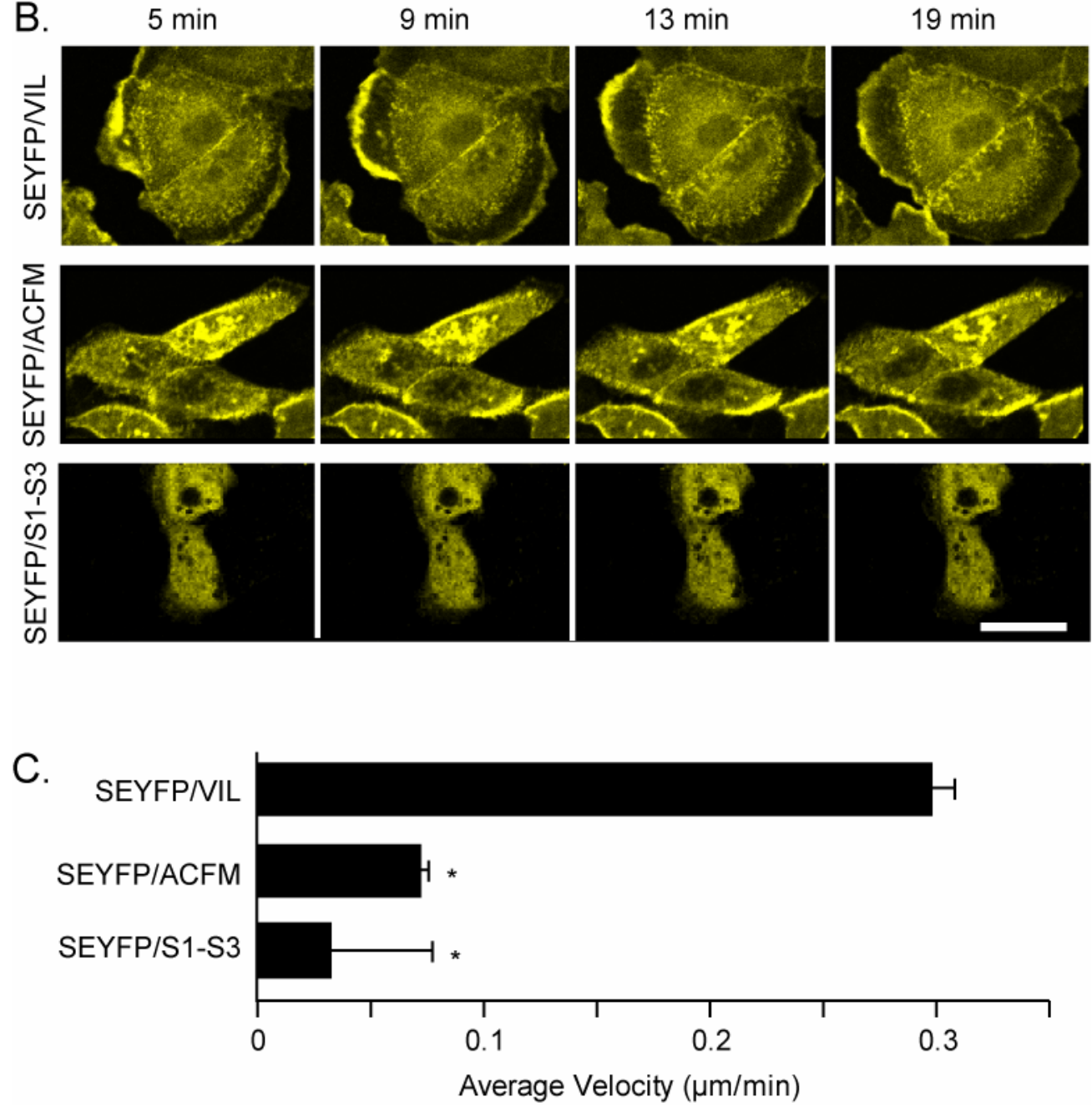
$(p<0.01, n=24)$. Likewise, mutation of all four amino-terminal phosphorylation sites (VIL/ANFM) significantly $(p<0.01, n=24)$ inhibited villin-induced cell migration, consistent with our previous report (Tomar et al., 2004). Together with our previous studies, these data demonstrate that multiple tyrosine phosphorylation sites contained within the core are required for villin's function in cell migration. To further explore the mechanisms underlying the observations described above, we generated a villin truncation mutant, S1-S3 (a.a. 1-338), that lacks the second half of the villin core (S4-S6) as well as the headpiece (S7), but retains the amino-terminal tyrosine phosphorylation sites. Full-length villin $(\mathrm{VIL})$, villin mutant lacking the carboxyl-terminal phosphorylation sites (ACFM), as well as S1-S3 truncation mutant were transiently expressed as SEYFP-tagged fusion proteins. All experiments were done $16 \mathrm{~h}$ post-transfection. MDCK Tet-Off cells transiently transfected with SEYFP/VIL, SEYFP/ACFM as well as SEYFP/S1-S3 were treated with LPA $(1 \mu \mathrm{M})$ or EGF $(50 \mathrm{ng} / \mathrm{ml})$ and time lapse images recorded every 2 min for 35 min. MDCK cells overexpressing full-length villin and treated with EGF $(50 \mathrm{ng} / \mathrm{ml})$ show association of villin with the lamellipodia (figure 3.8B). The association of villin with these cell surface structures was tightly coupled to the growth of the lamellipodia and the forward extension of the cell body. In contrast, MDCK Tet-Off cells overexpressing either SEYFP/ACFM or SEYFP/S1-S3 villin did not form lamellipodia and demonstrated little if any cell spreading during the 35 min of LPA/EGF treatment. In addition, unlike the full-length villin protein, the truncation mutant S1-S3 was localized diffusely to the cytosol (figure 3.8B). A similar observation was made in cells 
treated with LPA. Consistent with our previous report, time lapse images of MDCK Tet-Off cells transfected with a the villin mutant lacking all four aminoterminal phosphorylation sites, SEYFP/ANFM, show no lamellipod formation in response to EGF treatment (figure B.2A) (Tomar et al., 2004). We quantitated these morphological changes by measuring the rate of lamellipodial protrusion rate in MDCK Tet-Off cells expressing wild-type or mutant villin proteins essentially as described before (Rottner et al., 1999; Athman et al., 2003). MDCK cells expressing SEYFP/VIL demonstrated a significant increase in the rate of lamellipodia formation $(0.29 \pm 0.008 \mu \mathrm{m} / \mathrm{min})$ in MDCK cells expressing SEYFP/VIL compared to SEYFP/ACFM $(0.07 \pm 0.003 \mu \mathrm{m} / \mathrm{min})$ and SEYFP/S1S3 $(0.03 \pm 0.044 \mu \mathrm{m} / \mathrm{min}$ ) (figure $3.8 \mathrm{C})$. Similar observations were made in HeLa Tet-Off cells transiently transfected with these plasmids. These data suggest that optimal lamellipodia formation and maximum increase in cell migration following EGF/LPA treatment occurred in cells expressing full-length villin compared with cells expressing the amino-terminal phosphorylation site mutant, the carboxylterminal phosphorylation site mutant or the carboxyl-terminal truncation mutant of villin. Together these data confirm our observation that like the amino-terminal domain, the carboxyl-terminal domain of villin is also required for effective villininduced cell migration.

\subsubsection{Tyrosine phosphorylation of carboxyl-terminal domain of villin is} required for its association with PLC- $\gamma_{1}$. Since the carboxyl-terminal phosphorylation site mutants did not result in any significant changes in cell

shape or actin redistribution (figure 3.7, B.2), we speculated that the carboxyl- 
terminal phosphorylation sites in villin may determine its interaction with ligands that are crucial to cell migration. We have previously demonstrated that tyrosine phosphorylated villin associates with PLC- $\gamma_{1}$ and that PLC- $\gamma_{1}$ is a major binding partner of tyrosine phosphorylated villin in detergent-soluble fractions of cells (Khurana et al., 1997; Panebra et al., 2001). More recently using fluorescence resonance energy transfer (FRET) we have demonstrated the direct interaction of villin with the $\mathrm{SH} 2$ domains of PLC- $\gamma_{1}$ in living cells (Wang et al., Submitted). Thus, PLC- $\gamma_{1}$ is a physiologically significant ligand of tyrosine phosphorylated villin (Wang et al., Submitted). Further, we have demonstrated that this association of phospho-villin with PLC- $\gamma_{1}$ is required for its function in intestinal cell migration (Wang et al., Submitted). What was not reported in these studies was the specific structural determinants in villin that establish its association with PLC- $\gamma_{1}$. Based on these previous studies, we hypothesized that the $\mathrm{COOH}-$ terminal phosphorylation sites in villin may determine phospho-villin's association with PLC- $\gamma_{1}$. To test this hypothesis, we examined the co-immunoprecipitation of villin with PLC- $\gamma_{1}$ in MDCK Tet-Off cells ectopically expressing VIL/FL, VIL/ACFM, VIL/ANFM or VIL/AYFM. Using immunoprecipitated PLC- $\gamma_{1}$ we observed that tyrosine phosphorylated full-length villin associated with PLC- $\gamma_{1}$ in MDCK cells expressing wild-type villin protein (figure 3.9A). These data are consistent with our previous reports demonstrating the association of tyrosine phosphorylated villin with PLC- $\gamma_{1}$ (Khurana et al., 1997; Panebra et al., 2001; Wang et al., Submitted). In cells expressing VIL/ANFM PLC- $\gamma_{1}$, formed a complex with the mutant villin protein, indicating that amino-terminal phosphorylation sites 

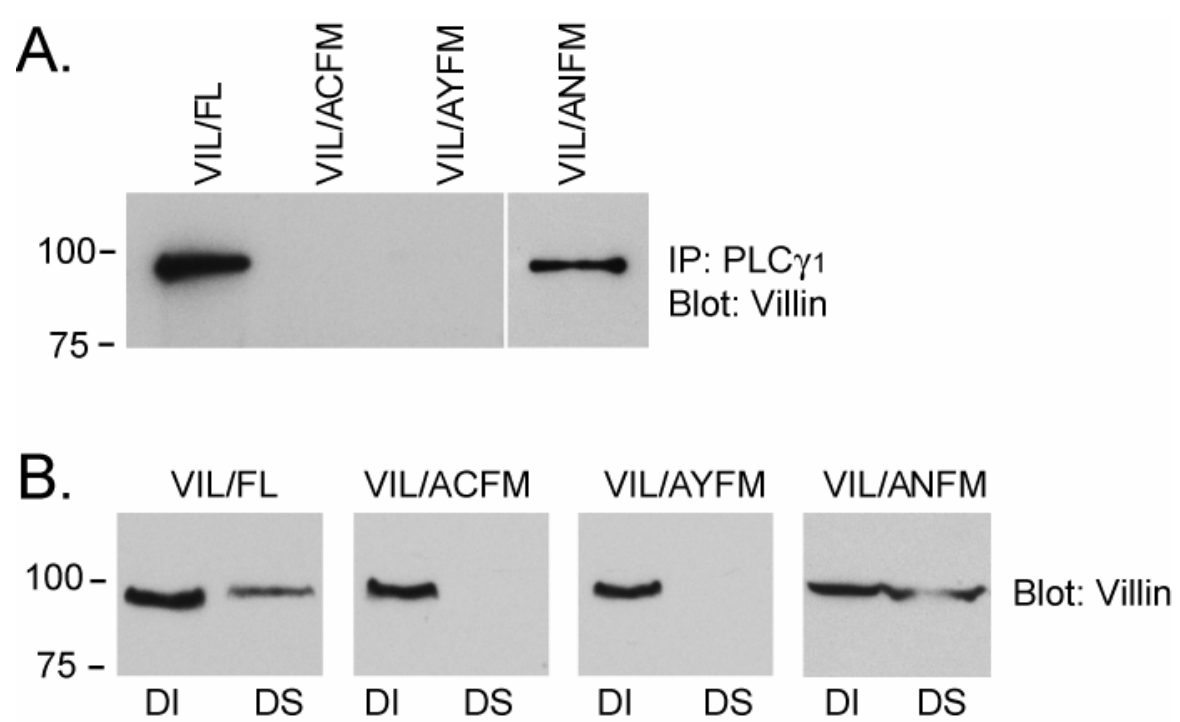

Figure 3.9 Collectively the carboxyl-terminal tyrosine phosphorylation sites are required for redistribution of villin to the detergent-soluble fraction and for its association with PLC- $\gamma_{1}$. (A) This figures shows co-immunoprecipitation of villin with PLC- $\gamma_{1}$ from the Triton X-100 soluble cell extracts. $\quad$ (B) Distribution of villin in detergent-soluble and insoluble cell fractions. Triton X-100-soluble fraction (DS) and Triton X-100-insoluble (DI) fractions from MDCK cells transfected with VIL/FL, VIL/ANFM VIL/ACFM or VIL/AYFM were immunoblotted with villin monoclonal antibodies. VIL/FL and VIL/ANFM distributes to the detergentsoluble and -insoluble fraction, while villin mutants, VIL/ACFM as well as VIL/AYFM distribute exclusively in the detergent-insoluble fraction. The western blots are representative of three other experiments with similar results. 
are not involved in PLC- $\gamma_{1}$ binding. In contrast, in cells expressing either VIL/ACFM or VIL/AYFM, PLC- $\gamma_{1}$ did not form a complex with villin. All individual point mutants of the carboxyl-terminal phosphorylation sites can associate with PLC- $\gamma_{1}$ (figure B.2B). It may be noted that for these co-immunoprecipitation studies PLC- $\gamma_{1}$ was immunoprecipitated from the Triton X-100-soluble fraction of cells. These data show that collectively the carboxyl-terminal tyrosine phosphorylation sites determine villin's association with PLC- $\gamma_{1}$. In concurrence with these immunoprecipitation studies, analysis of the detergent-soluble and -insoluble pools indicated that wild-type villin protein was distributed both in the Triton-soluble and Triton-insoluble fractions of the cell, as reported earlier (Khurana et al., 1997). Likewise, VIL/ANFM protein was distributed both in the detergent-soluble and -insoluble fractions of the cell. In contrast, in cells expressing either of the phosphorylation site mutants, VIL/ACFM or VIL/AYFM the villin mutant proteins were almost exclusively distributed to the Triton $\mathrm{X}-100$ insoluble fraction and none of the mutant proteins were associated with detergent-soluble cell fraction (figure 3.9B). This is noteworthy, since we have previously demonstrated that the majority of the PLC- $\gamma_{1}$ present in ileal villus brush border is associated with the Triton-soluble fraction and not the detergentinsoluble fraction (Khurana et al., 1997). Further, we have also shown that the tyrosine phosphorylated villin associates with PLC- $\gamma_{1}$ in the detergent-soluble cell fraction (Khurana et al., 1997). Taken together, these data suggest that the carboxyl-terminal tyrosine phosphorylation sites in villin determine villin's distribution to the detergent-soluble cell fraction, where phosphorylation of these 
sites allows villin to form a complex with PLC- $\gamma_{1}$ which is required for villininduced increase in cell migration (figure 3.10). While studying the SEYFP/S1S3 mutant that lacks the second half of villin core (S4-S6) as well as head piece (S7), we made an interesting observation. We determined that longer transfection times $(>20 \mathrm{~h})$ resulted in significant cell death in HeLa/MDCK Tet-Off cells expressing the truncation mutant $\mathrm{S} 1-\mathrm{S} 3$ as noted by cell rounding and loss of fluorescence, increase in TUNEL positive cells, chromatin condensation and staining with Hoechst 33258 (figure B.3). For the same reason, stable cell lines expressing S1-S3 could not be established for these studies. We speculate that like gelsolin, the S1-S3 domain of villin results in unregulated actin severing and apoptotic cell death (Kothakota et al., 1997). This may be important to villin's role as an anti-apoptotic protein and if villin is a substrate for caspases and/or other proteases, the cleavage of villin into this fragment may have a regulatory function (Wang, Y. and Khurana, S., unpublished observation). Hence, the carboxyl-terminal domain of villin may also be required to maintain a biologically relevant conformation of villin that may be important to its functions as an antiapoptotic protein. Most importantly, the data presented in this study demonstrate that the carboxyl terminal half of the villin core is not redundant as has been suggested by some, but essential to villin's function in cell migration and perhaps cell death. 


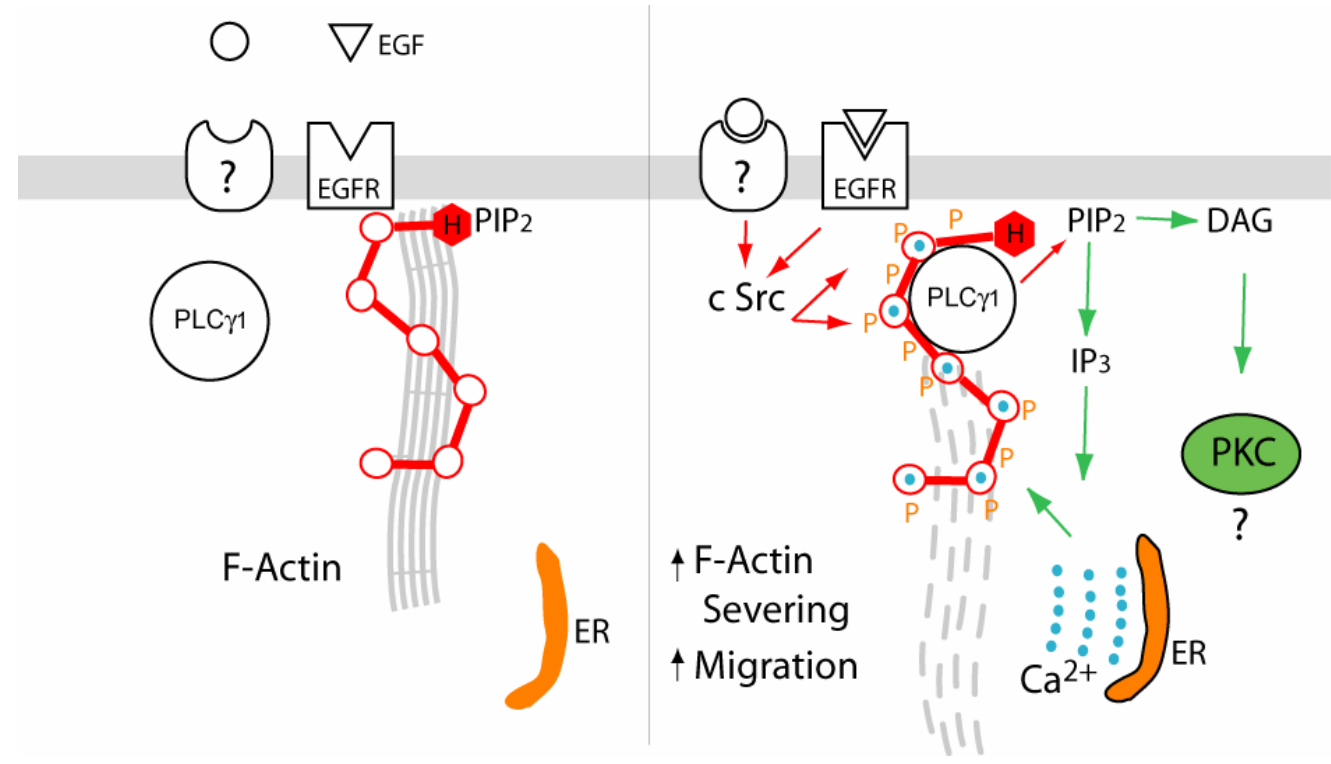

Figure 3.10 Schematic representation of the role of the carboxyl-terminal tyrosine phosphorylation sites in villin-induced cell migration. The panel on the left shows resting cells in which villin associates with $\mathrm{PIP}_{2}$ at the plasma membrane. In response to receptor activation including EGF treatment, villin is tyrosine phosphorylated (by c-src kinase) and dissociates from $\mathrm{PIP}_{2}$ and binds PLC- $\gamma_{1}$ through its carboxyl-terminal phosphorylation sites. The association of villin with PLC- $\gamma_{1}$ at the plasma membrane results in the hydrolysis of $\mathrm{PIP}_{2}$ into diacylglycerol (DAG) and inositol-1,4,5-trisphosphate $\left(\mathrm{IP}_{3}\right)$ (Wang et al., Submitted). $\quad \mathrm{IP}_{3}$ releases $\mathrm{Ca}^{2+}$ from intracellular stores such as the endoplasmic reticulum (ER). Both tyrosine phosphorylation as well as increase in intracellular calcium induce actin-severing by villin resulting in actin remodeling and change in cell morphology and increase in cell migration. 


\subsection{Discussion}

Metastases is the most lethal property of cancer cells and critical to this property is the increased migratory state of tumor cells. The current thinking is that cancer cells switch to a more motile state thus enhancing the metastatic potential of a tumor cell. The molecular mechanisms that propel invasive growth and metastases are also found during embryonic development and in adult tissue maintenance such as wound repair. Most human cancers originate from epithelial cells and include the major killers namely colon, breast and lung cancer. Epithelial cell migration differs from that of fibroblasts in that epithelia migrate as sheet such as during wound closure or as a tube such as during kidney development in vertebrates. More recent studies have established that examining epithelial cell migration in the context of proteins that are uniquely expressed in epithelial cells including the actin binding protein villin is important to our understanding of this rather complex process (Athman et al., 2003; Wang et al., Submitted). We have demonstrated that overexpression of villin enhances cell migration (Tomar et al., 2004). Likewise Athman et al have demonstrated that villin enhances hepatocyte growth factor induced actin remodeling in epithelial cells (Athman et al., 2003). Tyrosine phosphorylation of villin is essential to its function in cell migration (Athman et al., 2003; Tomar et al., 2004). We have previously reported that three tyrosine residues (Y60, Y81 and Y256) in the amino-terminal domain of villin are required for villin-induced increase in cell migration (Tomar et al., 2004). Phosphorylation at these three sites was shown to 
regulate the intracellular distribution of villin, the F-actin redistribution and remodeling as well as villin-induced increase in cell migration (Tomar et al., 2004). In this study we determined that in addition to these three amino-terminal residues, the carboxyl-terminal tyrosine phosphorylation sites of villin are equally important to its function in cell migration. While the tyrosine phosphorylation sites in the amino-terminal half regulate villin-induced cell migration by regulating the actin reorganization and cell morphology, the tyrosine phosphorylation sites in the carboxyl-terminal half regulate villin's ligand-binding properties, namely its association with PLC- $\gamma_{1}$. Mutation of all six carboxyl-terminal phosphorylation sites in villin prevents the association of villin with PLC- $\gamma_{1}$. The role of PLC- $\gamma_{1}$ in cell migration is undisputed. Overexpression of PLC- $\gamma_{1}$ has been shown to result in malignant transformation and has been implicated in the pathophysiology of tumorigenesis (Soderquist et al., 1992; Nomoto et al., 1995; Chang et al., 1997; Smith et al., 1998). Overexpression of PLC- $\gamma_{1}$ is associated with progression of colorectal tumors from normal mucosa to adenoma and then to carcinoma (Park et al., 1994). Growth factor mediated PLC- $\gamma_{1}$ signaling is also required for enhanced cell motility (Chen et al., 1994; Polk, 1998; Wells, 2000). For instance, activation of PLC- $\gamma_{1}$ downstream of HGF receptor activation is required for the morphogenetic effects of HGF during epithelial-mesenchymal transition (Ponzetto et al., 1994; Gual et al., 2000). Redistribution of PLC- $\gamma_{1}$ to the leading edge and the activation of the phosphoinositide signal cascade at the leading edge is likewise well documented (Chang et al., 1997; Chou et al., 2002; Piccolo et al., 2002). Our study suggests that villin keeps the signal equilibrium of actin 
cytoskeleton organization, cell morphology and cell migration by utilizing distinct domains and tyrosine phosphorylation sites within these domains in a differential and cooperative way.

In this study we determined that villin mutant lacking the carboxyl-terminal phosphorylation sites does not associate with the detergent-soluble fraction of the cell thus preventing the association of phospho-villin with PLC- $\gamma_{1}$. Likewise, time-lapse video demonstrate that mutation of the carboxyl-terminal phosphorylation sites prevents villin's localization to the lamellipodia. A very important step in cell locomotion is the development of a polarized phenotype with the formation of a leading edge in the direction of cell movement. It has been suggested that in addition to PI3-kinase, PLC- $\gamma_{1}$ also determines the polarized phenotype of a motile cell by its recruitment to the leading edge thus regulating the first step of cell migration, namely the formation of a protrusion (Chang et al., 1997; Chou et al., 2002; Piccolo et al., 2002). In fact, such an observation has been made with cofilin, where inhibition of PLC inhibited the effects of cofilin during the early stages of cell migration by delaying the initiation of lamellipodia formation and inhibiting the ability of the cells to sense EGF gradients (Mouneimne et al., 2004). In these studies, the authors determined that both cofilin and PLC activation were required for the initial but not late changes in the generation of free barbed ends, thus regulating cell migration. Our own studies with PLC- $\gamma_{1}{ }^{-/-}$and PLC- $\gamma_{1}{ }^{+/+}$cells have demonstrated that overexpression of villin and villin-induced increase in cell migration requires PLC$\gamma_{1}$ (Wang et al., Submitted). Likewise, we have demonstrated that down 
regulation of PLC- $\gamma_{1}$ using small, interfering, hairpin RNA (siRNA) prevents villininduced increase in cell migration (Wang et al., Submitted). Together, these studies suggest that villin-induced increase in cell migration is likely regulated by a rapid increase in actin polymerization at the leading edge which is enhanced by tyrosine phosphorylation of villin and by the catalytic activation of PLC- $\gamma_{1}$ by phospho-villin. Thus, the synergistic interaction between tyrosine phosphorylated villin and PLC- $\gamma_{1}$ may drive the EGF stimulated lamellipod formation seen in our studies. Together with our previous reports, data presented in this study show that tyrosine phosphorylation sites in the carboxyl-terminal domain of villin are important for villin's ligand binding properties, specifically for its association with PLC- $\gamma_{1}$, thus regulating villin-induced cell migration (figure 3.10) (Panebra et al., 2001).

Another interesting observation made in this study was the significance of the carboxyl terminal domains of villin in maintaining a functionally relevant protein conformation. Transient expression of S1-S3 fragment of villin in HeLa/MDCK Tet-Off cells resulted in cell death (figure B.2). These studies suggest that the S1-S3 fragment of villin might be functionally similar to the S1S3 fragment of gelsolin, which has been shown to be pro-apoptotic resulting in cell death (Kothakota et al., 1997; Azuma et al., 2000). Further, these studies suggest that the carboxyl-terminal half of the villin core may be functionally similar to gelsolin domains S4-S6, in that these domains may be required to maintain an autoinhibited conformation that prevents unregulated actin depolymerization. It is also likely that releasing this conformation may be 
biologically relevant to the functions of these proteins in vivo. While a caspase-3 cleavage site has been identified in gelsolin that generates the pro-apoptotic S1S3 fragment, a similar caspase cleavage site or caspase-mediated cleavage of villin is lacking, even though the villin fragment S1-S3 appears to be apoptotic (Wang Y., and Khurana, S., unpublished observation) (Burtnick et al., 2004). The pro-apoptotic functions of these two proteins could thus be regulated differently as well. For instance gelsolin could be a substrate for caspase-3 while villin could be a substrate for other proteases. Such proteolytic cleavage of villin has been reported in enteric cells infected with Entamoeba histolytica (Lauwaet et al., 2003). It may also be noted that in villin-gelsolin chimeras, where the S4S6 domains of villin were substituted with the second half of the gelsolin core, failed to function like full-length villin protein, suggesting that while these two proteins share significant structural and functional homology, they are not identical (Finidori et al., 1992). This lends support to our view that proteins of this family may have overlapping and yet distinct function(s) in tissues where more than one member of this family is expressed such as in the intestine and kidney.

Our in vitro studies allowed us to identify the carboxyl-terminal phosphorylation sites in villin. In addition, we determined that phosphorylation of multiple carboxyl-terminal domain phosphorylation sites could regulate actinnucleation by villin, while phosphorylation of Y286 enhanced actindepolymerization by villin. Further, we determined that tyrosine phosphorylation of villin does not regulate its actin capping activity. Together with our previous studies, these data confirm our observation that tyrosine phosphorylation 
regulates all but one actin regulatory activity of villin (Zhai et al., 2001; Zhai et al., 2002; Tomar et al., 2004). While the carboxyl-terminal phosphorylation sites regulated actin kinetics in vitro, mutation of these sites did not result in any significant change in actin redistribution in the cell. The significance of these changes is unclear at this point.

In addition to revealing new tyrosine phosphorylation sites as well as identifying all phosphoryable tyrosine residues in villin, this study established the domain functions of villin by investigating the ability of a series of mutants of villin to rescue actin organization, cell morphology, and cell migration. These studies determined that the amino terminal half of the core regulates actin reorganization and cell shape while the entire villin core is required for cell migration. In this report we demonstrate for the first time the functional relevance of the carboxylterminal tyrosine phosphorylation sites in villin, namely: in the distribution of villin to the detergent soluble fraction; in the association of villin with its ligand PLC- $\gamma_{1}$ in this fraction; in growth factor induced lamellipodia formation, hence in defining the directionality of a polarized motile cell; and in the synergistic interaction with PLC- $\gamma_{1}$ to initiate the early steps involved in actin polymerization and assembly of the lamellipodia in a moving cell. Elucidating the molecular mechanisms linking the actin regulatory activities modified by phosphorylation of these sites with villin's function(s) will be the subject of future studies. 


\section{Chapter 4}

\section{Discussion}




\subsection{Tyrosine phosphorylation of villin and actin kinetics}

The work done in our lab has demonstrated that villin gets phosphorylated both in vitro and in vivo (Khurana et al., 1997; Panebra et al., 2001). It was also demonstrated that tyrosine phosphorylation of villin plays a vital role in the biological properties of villin (Khurana et al., 1997; Panebra et al., 2001; Zhai et al., 2001; Zhai et al., 2002). The next challenge was to identify the sites of phosphorylation and to determine the role of the identified phosphorylation sites in villin's functions. The initial approach used to identify the sites of phosphorylation was by mass spectrometry. However, this approach yielded a lot of false positive results and the identified phosphorylation sites could not be validated by mutational analysis. Since, the results obtained using mass spectrometry would have required confirmation by mutational analysis, we elected to identify the phosphorylation sites using the same approach namely, mutational analysis. Tyrosine phosphorylated villin was generated in vitro using a unique strategy employing Epicurian coli TKX1 cells. TKX1 cells carry a plasmid with the elk tyrosine kinase (tk) gene controlled by the trp promoter. The elk tyrosine kinase has broad specificity and has been shown to tyrosine phosphorylate a number of eukaryotic proteins expressed in Epicurian coli TKX1 cells (Chin et al., 1996; Kozlowski et al., 1998). Human villin cDNA was cloned in the prokaryotic expression vector pGEX-2T and a two-step protocol involving the expression of human villin gene (by addition of IPTG) followed by the expression 
of the tk gene [by addition of 3- $\beta$-indoleacrylic acid (IAA)], in Epicurian coli TKX1 cells resulted in generation of GST-tagged tyrosine-phosphorylated villin (VILT/WT). We used site-directed mutagenesis to identify the tyrosine phosphorylation sites in villin. We generated 10 truncations and approximately 50 individual point mutants (tyrosine to phenylalanine) of villin. A loss of signal in quantitative western blots, probed with a phospho-tyrosine specific antibody (PY20), in individual $Y$ to $F$ point mutants allowed us to identify putative tyrosine phosphorylation sites in villin (Zhai et al., 2002). Using this approach four phosphorylation sites were identified in the amino-terminus of human villin, namely Y-46, $-60,-81$, and -256 . The identified sites were also substrates of csrc kinase in vitro (Zhai et al., 2002). Interestingly, we noted that mutation of the four amino terminus tyrosine phosphorylation sites (to phenylalanine) still resulted in villin phosphorylation, indicating the presence of additional phosphorylation sites in the carboxyl-terminus of villin (Tomar et al., 2006). I used the same approach (as described above) to identify six additional tyrosine phosphorylation sites in the carboxyl-terminus of human villin, namely, Y-286, $-324,-461,-555,-604$ and -725 . All ten identified tyrosine phosphorylation sites are well conserved across species. Y-46, $-256,-286,-324,-461,-555$, and -604 are also well conserved in other proteins of the villin family, including gelsolin, adseverin and cap $G$ (table 4.1). To confirm that all the phosphorylation sites in villin had been identified we generated a villin mutant with all ten identified tyrosine residues mutated to phenylalanine (VIL/AYFM). VIL/AYFM was not phosphorylated when expressed as a recombinant protein 


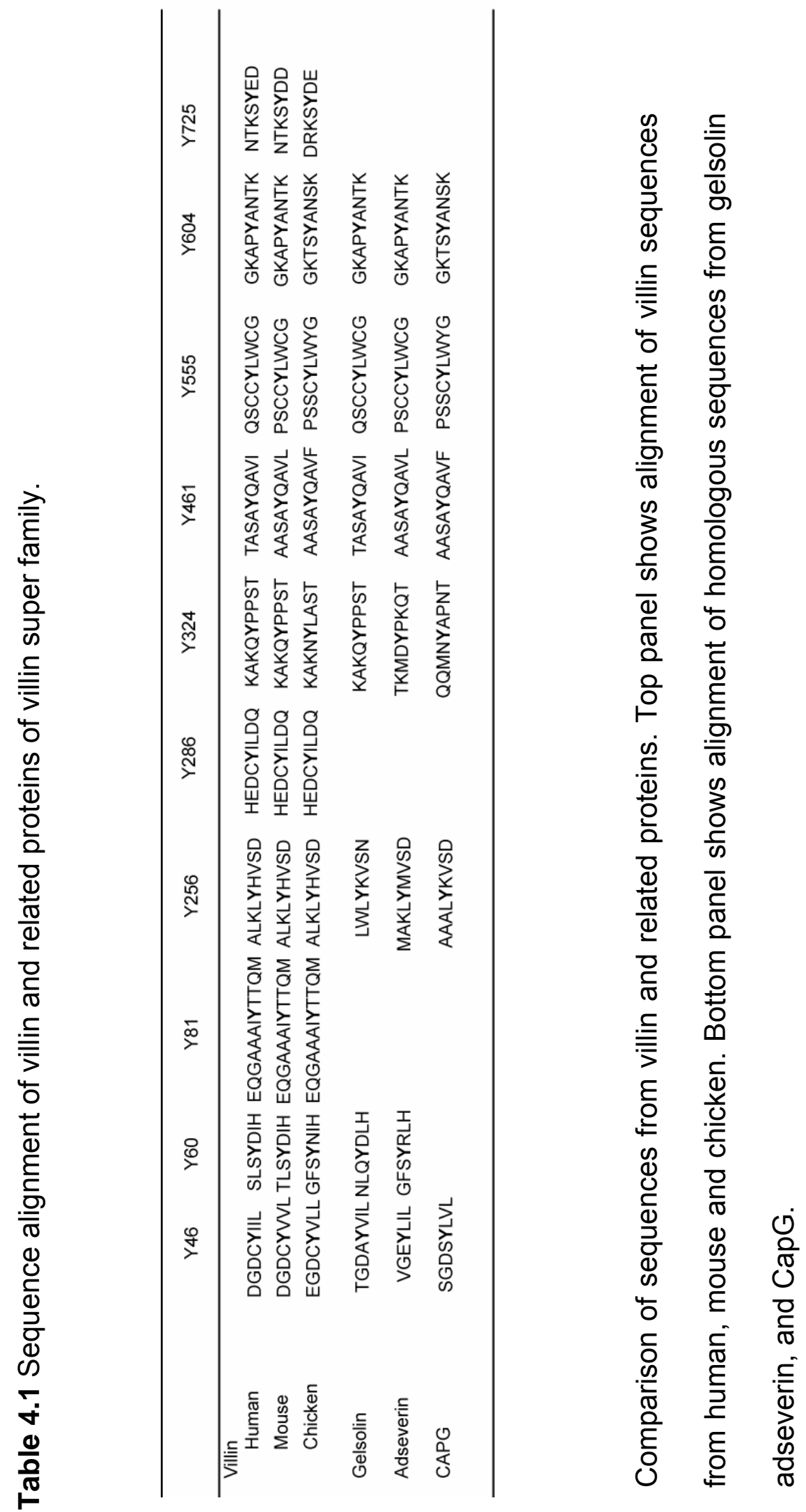


using Epicurian coli TKX1 cells. VIL/AYFM was also not phosphorylated when expressed in HeLa and MDCK Tet-off cells (chapter 3).

The next task was to map phosphorylation at each of these sites with villin's actin regulatory functions. For these studies we used muscle actin as a source of pyrene labeled G-actin or F-actin. Each of the ten identified phosphorylation sites were mutated individually (to phenylalanine) in full-length villin and the role of these sites on villin's actin modifying functions was determined in vitro. From these studies we determined that tyrosine phosphorylation of villin enhanced actin-severing, inhibited -nucleation and -bundling functions of villin but had no effect on the actin-capping activity of villin. Further, we established that phosphorylation at residues 46, 60 and 286 enhanced the actin-severing activity of villin. Mutation of any one of the ten identified phosphorylation sites had no effect on the actin nucleating function of phospho-villin. Thus, two or more phosphorylation sites regulate the actinnucleating function of villin (table 4.2).

\subsection{A summary of villin's role in cell migration}

Cell migration is regulated by correct spatial and temporal organization of actin filaments, which is determined largely by the actin binding proteins. Villin is one of the most versatile actin binding protein in epithelial cells, as it can regulate 
Table 4.2 Tyrosine phosphorylation of villin and its role in actin kinetics.

\begin{tabular}{ccccc}
\hline $\begin{array}{c}\text { Tyrosine } \\
\text { phosphorylation } \\
\text { sites }\end{array}$ & Nucleationa & Severing & Capping & Bundling \\
\hline 46 & + & + & - & N.D. \\
60 & + & + & - & N.D. \\
81 & + & - & - & N.D. \\
256 & + & - & - & N.D. \\
286 & + & + & - & N.D. \\
324 & + & - & - & N.D. \\
461 & + & - & - & N.D. \\
555 & + & - & - & N.D. \\
604 & + & - & - & N.D. \\
725 & + & - & - & N.D. \\
\hline
\end{tabular}

Actin-severing is enhanced by phosphorylation at residues, 46,60 and $286 .{ }^{a}$ Two or more of the identified tyrosine residues regulate actin-nucleation by villin. Tyrosine phosphorylation does not regulate actin-capping activity of villin. ${ }^{\mathrm{b}}$ Tyrosine phosphorylation of villin inhibits actin-bundling but the specific residues regulating this function have not been identified. 
actin-nucleation, -severing, -capping and -bundling. As discussed earlier, (chapters 2 and 3 ) overexpression of villin in intestinal and renal epithelial cells enhanced the rate of both basal as well as growth factor induced cell migration. Further, we demonstrated that tyrosine phosphorylation of villin was required for villin's function in cell migration. Tyrosine phosphorylation of villin as well as villininduced cell migration was also significantly inhibited by overexpression of a dominant negative mutant of c-src kinase, confirming the significance of tyrosine phosphorylated villin in the regulation of cell migration (chapter 2). As summarized in table 4.2, we have identified 10 tyrosine residues in villin that are phosphorylated. Interestingly all but one of the phosphorylation sites enhanced villin-induced cell migration ( $\mathrm{Y}-46$ to $-\mathrm{F}$ had no effect). The amino-terminus phosphorylation sites (Y-60, -81 and -256) regulated villin's intracellular distribution, actin reorganization and changes in cell morphology thus, regulating villin-induced cell migration (chapter 2). In contrast, phosphorylation of the carboxyl-terminus sites (Y-286, -324, -461, -555, -604, and -725) had no effect on villin-induced changes in actin reorganization or cell morphology, but were found to be critical for villin's interaction with its ligand PLC- $\gamma_{1}$. The role of PLC- $\gamma_{1}$ in cell migration is well documented (chapter 3). The role of all ten identified tyrosine phosphorylation sites in villin-induced cell migration has been summarized in table 4.3 .

As discussed in chapter 1 , the formation and growth of lamellipodia in a motile cell requires actin polymerization. Severing of existing filaments, uncapping of existing filaments or de novo nucleation can all enhance actin 
Table 4.3 Tyrosine phosphorylation of villin and its role in cell migration.

\begin{tabular}{ccccc}
\hline $\begin{array}{c}\text { Tyrosine } \\
\text { phosphorylation } \\
\text { sites }\end{array}$ & $\begin{array}{c}\mathrm{PLCy}_{1} \\
\text { association }\end{array}$ & $\begin{array}{c}\text { Cell } \\
\text { shape }\end{array}$ & $\begin{array}{c}\text { F-actin } \\
\text { reorganization }\end{array}$ & Cell migration \\
\hline${ }^{\mathrm{b}} 46$ & - & - & - & + \\
60 & - & + & + & + \\
81 & - & + & + & + \\
256 & - & + & + & + \\
286 & + & - & - & + \\
324 & + & - & - & + \\
461 & + & - & - & + \\
555 & + & - & - & + \\
604 & + & - & - & + \\
725 & + & - & - & + \\
\hline
\end{tabular}

The role of all ten identified tyrosine phosphorylation sites in cell migration is summarized. ${ }^{a}$ More than one tyrosine phosphorylation site is involved in interaction with PLC- $\gamma_{1}$. ${ }^{b}$ Mutation of $\mathrm{Y}-46$ to $-\mathrm{F}$ had no effect on cell migration, however mutation of $\mathrm{Y}-46$ to $-\mathrm{E}$ inhibited cell migration (Chapter 2). 
polymerization. Tyrosine phosphorylated villin can sever F-actin to provide new actin nuclei thus enhancing actin polymerization. Tyrosine phosphorylation of villin does not regulate the capping activity of villin and inhibits the nucleating activity of villin. Hence, we speculate that severing of actin filaments by villin can provide new nuclei for elongation at the leading edge. As discussed in chapter 1, the current thinking is that de novo nucleation at the leading edge, primarily by Arp2/3 complex, is crucial for generation of new filaments and cell migration. However, some recent studies have suggested that a synergy between actinsevering proteins like cofilin and Arp2/3 is required for optimal generation of barbed ends at the leading edge of moving cells (Chan et al., 2000; Bailly et al., 2001). The function-blocking antibodies directed against either cofilin or Arp2/3 significantly decreased barbed-end generation and cell protrusion (Chan et al., 2000; Bailly et al., 2001). Further, it has also been demonstrated that the severing activity of cofilin and nucleating activity of Arp2/3 alone, caused only a modest increase in actin polymerization. However, together they significantly enhanced actin polymerization, suggesting a role for both proteins in cell migration (Ichetovkin et al., 2000). A similar argument has been made for the interaction between the Arp2/3 complex and gelsolin in platelets and fibroblasts, in which new actin nuclei generated by the severing activity of gelsolin has been proposed to be necessary for the activity of the Arp2/3 complex (Falet et al., 2002). These studies indicate that the severing activity of actin-binding proteins like cofilin, gelsolin and by analogy villin may be necessary for optimal actin nucleation by the Arp $2 / 3$ complex. It is suggested that the actin nucleating activity 
of the Arp $2 / 3$ complex is enhanced because the severing activity of proteins such as cofilin and gelsolin generates new barbed ends, which are the preferred sites for binding of the Arp2/3 complex, compared with older, ADP-containing filaments (DesMarais et al., 2005). Hence, depending on the cell type, different actin severing proteins may act synergistically with $\operatorname{Arp} 2 / 3$ to regulate cell migration. This synergistic interaction between actin severing proteins and Arp2/3 is also important for providing directionality, as actin severing and generation of new nuclei at the leading edge would determine the sites of dendritic nucleation and therefore the site of membrane protrusion (DesMarais et al., 2005).

For future studies, it would be interesting to examine the role of Arp2/3 complex in villin-induced cell migration, to ask such questions as: can villin regulate cell migration in cells where Arp2/3 is inactivated? In the absence of Arp2/3 activity do villin expressing cells form primarily lamellipodia (cross-linked actin) or filopodia (bundled actin)? If not, then is there a synergy between Arp2/3 complex and villin during epithelial cell migration? Is villin targeted to the leading edge before or after Arp2/3? Does the role of villin in phosphoinositide signalling play any role in activation of Arp2/3 complex and its targeting to the leading edge? Similarly it would also be important to examine the role of Rho GTPases, specifically Rac and Cdc42 in villin induced cell migration. The answers to these and similar questions would help establish the precise role that villin plays in concert with other proteins at the leading edge during epithelial cell migration. 


\subsection{Tyrosine phosphorylation of villin: Leading the way in epithelial cell migration}

In chapter 1 the original steady-state "dendritic model" has been discussed. In this model actin severing proteins like cofilin, gelsolin or villin are proposed to function exclusively as actin-recycling factors, depolymerizing filaments to yield G-actin, but not as severing proteins that could generate new nuclei for filament assembly during cell migration. Moreover, the model does not take into account the synergistic interactions between actin-severing proteins and Arp2/3 complex, which are now reasonably well established. Hence, the steadystate "dendritic model" fails to justify the role of actin-severing proteins in cell migration. More recently a new model of cell migration has been described which takes into consideration the synergistic interaction of actin-severing proteins with Arp2/3 complex and is called the "stimulated protrusion model" (DesMarais et al., 2005). This model proposes that the severing activity of actin-binding proteins at the leading edge determines the site of barbed end formation hence, determining the site of dendritic nucleation and therefore the site of membrane protrusion (figure 4.1). It is therefore reasonable to speculate that since villin is the most abundant actin-severing protein of its family in intestinal and renal epithelial cells, and the only actin severing protein expressed in microvilli of epithelial cells, it could regulate cell migration by generating new actin nuclei at the leading edge. These severed actin filaments could then act as sites of dendritic nucleation, thus determining the site of membrane protrusion. 
1

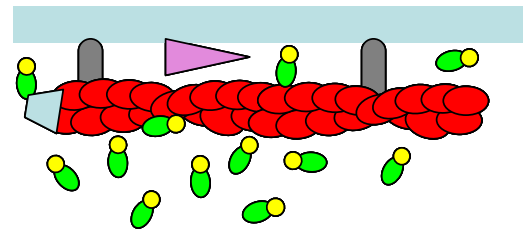

3
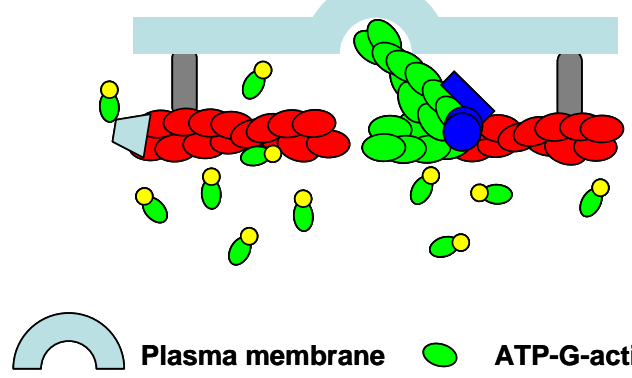

○

Plasma membrane

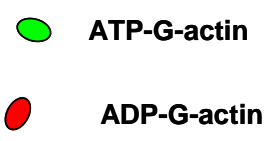

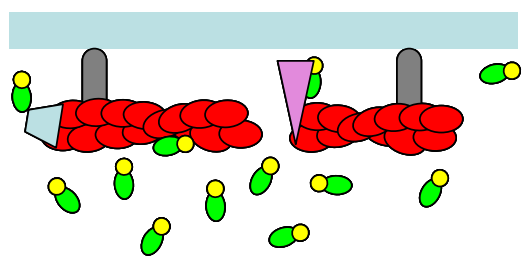

4

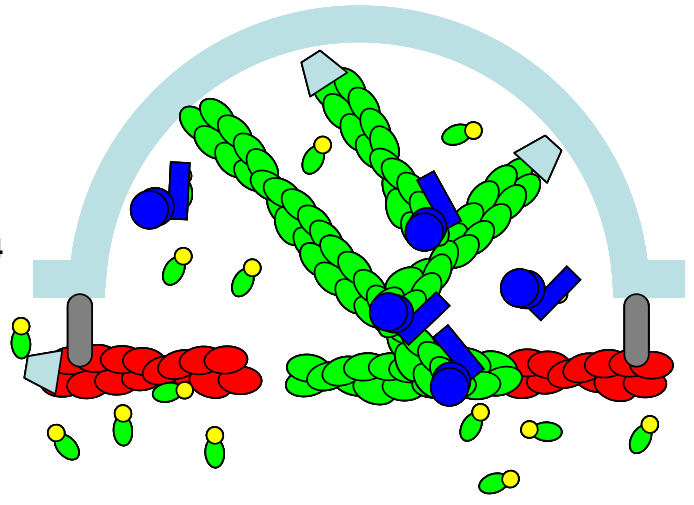

Arp2/3 complex

Capping protein

ABP

Figure 4.1 Stimulated protrusion model. (1) Under resting condition the actin severing protein is not activated. (2) The localized activation of actin severing protein generates free barbed end. (3) Profilin bound ATP-G-actin is added to the barbed end, generating ATP actin cap, where activated Arp2/3 complex binds and initiates branch formation. (4) Rapid growth occurs at the barbed end of actin filaments which pushes the membrane forward. 
Hence, like cofilin, villin could serve as a dynamic component of the steering wheel in a moving cell. 


\section{List of References}


Abercrombie, M., Heaysman, J.E., and Pegrum, S.M. (1972). Locomotion of fibroblasts in culture v. Surface marking with concanavalin A. Exp. Cell Res. 73, 536-539.

Aizawa, H., Sutoh, K., and Yahara, I. (1996). Overexpression of cofilin stimulates bundling of actin filaments, membrane ruffling, and cell movement in Dictyostelium. J. Cell Biol. 132, 335-344.

Albers, T.M., Lomakina, I., and Moore, R.P. (1996). Structural and functional roles of cytoskeletal proteins during repair of native guinea pig intestinal epithelium. Cell Biol. Int. 20, 821-830.

Anderson, R.D., Haskell, R.E., Xia, H., Roessler, B.J., and Davidson, B.L. (2000). A simple method for the rapid generation of recombinant adenovirus vectors. Gene Ther. 7, 1034-1038.

Arora, P.D., and McCulloch, C.A. (1996). Dependence of fibroblast migration on actin severing activity of gelsolin. J. Biol. Chem. 271, 20516-20523.

Arpin, M., Pringault, E., Finidori, J., Garcia, A., Jeltsch, J.M., Vandekerckhove, J., and Louvard, D. (1988). Sequence of human villin: a large duplicated domain homologous with other actin-severing proteins and a unique small carboxyterminal domain related to villin specificity. J. Cell. Biol. 107, 1759-1766.

Athman, R., Fernandez, M.I., Gounon, P., Sansonetti, P., Louvard, D., Philpott, D., and Robine, S. (2005). Shigella flexneri infection is dependent on villin in the mouse intestine and in primary cultures of intestinal epithelial cells. Cell Microbiol. 7, 1109-1116.

Athman, R., Louvard, D., and Robine, S. (2003). Villin enhances hepatocyte growth factor-induced actin cytoskeleton remodeling in epithelial cells. Mol. Biol. Cell 14, 4641-4653.

Azuma, T., Koths, K., Flanagan, L., and Kwiatkowski, D. (2000). Gelsolin in complex with phosphatidylinositol 4,5-bisphosphate inhibits caspase-3 and -9 to retard apoptotic progression. J. Biol. Chem. 275, 3761-3766.

Bacchi, C.E., and Gown, A.M. (1991). Distribution and pattern of expression of villin, a gastrointestinal-associated cytoskeletal protein, in human carcinomas: a study employing paraffin-embedded tissue. Lab. Invest. 64, 418-424.

Bailly, M., Ichetovkin, I., Grant, W., Zebda, N., Machesky, L.M., Segall, J.E., and Condeelis, J. (2001). The F-actin side binding activity of the Arp2/3 complex is essential for actin nucleation and lamellipod extension. Curr. Biol. 11, 620-625.

Barthod, F. (1994). Effects of ischemia and revascularization on the epithelium of the small intestine: study on swine. J. Chir. (Paris) 131, 221-235. 
Boyer, B., Valles, A.M., and Thiery, J.P. (1996). Model systems of epitheliummesenchyme transitions. Acta. Anat. (Basel) 156, 227-239.

Bretscher, A., and Weber, K. (1980). Villin is a major protein of the microvillus cytoskeleton which binds both $G$ and $F$ actin in a calcium-dependent manner. Cell 20, 839-847.

Brown, D., Lee, R., and Bonventre, J.V. (1997). Redistribution of villin to proximal tubule basolateral membranes after ischemia and reperfusion. Am. J. Physiol. 273, F1003-1012.

Bryant, P.J. (1999). Filopodia: fickle fingers of cell fate? Curr. Biol. 9, R655-657.

Burtnick, L.D., Koepf, E.K., Grimes, J., Jones, E.Y., Stuart, D.I., McLaughlin, P.J., and Robinson, R.C. (1997). The crystal structure of plasma gelsolin: implications for actin severing, capping, and nucleation. Cell 90, 661-670.

Burtnick, L.D., Urosev, D., Irobi, E., Narayan, K., and Robinson, R.C. (2004). Structure of the $\mathrm{N}$-terminal half of gelsolin bound to actin: roles in severing, apoptosis and FAF. Embo J. 23, 2713-2722.

Calalb, M.B., Polte, T.R., and Hanks, S.K. (1995). Tyrosine phosphorylation of focal adhesion kinase at sites in the catalytic domain regulates kinase activity: a role for Src family kinases. Mol. Cell. Biol. 15, 954-963.

Carlier, M.F., and Pantaloni, D. (1997). Control of actin dynamics in cell motility. J. Mol. Biol. 269, 459-467.

Carlier, M.F., Pantaloni, D., and Korn, E.D. (1984). Evidence for an ATP cap at the ends of actin filaments and its regulation of the F-actin steady state. J. Biol. Chem. 259, 9983-9986.

Cary, L.A., Chang, J.F., and Guan, J.L. (1996). Stimulation of cell migration by overexpression of focal adhesion kinase and its association with Src and Fyn. J. Cell. Sci. 109, 1787-1794.

Chan, A.Y., Bailly, M., Zebda, N., Segall, J.E., and Condeelis, J.S. (2000). Role of cofilin in epidermal growth factor-stimulated actin polymerization and lamellipod protrusion. J. Cell. Biol. 148, 531-542.

Chang, J.S., Noh, D.Y., Park, I.A., Kim, M.J., Song, H., Ryu, S.H., and Suh, P.G. (1997). Overexpression of phospholipase C-gamma1 in rat 3Y1 fibroblast cells leads to malignant transformation. Cancer Res. 57, 5465-5468.

Chellaiah, M., Kizer, N., Silva, M., Alvarez, U., Kwiatkowski, D., and Hruska, K.A. (2000). Gelsolin deficiency blocks podosome assembly and produces increased bone bass and strength. J. Cell Biol. 148, 665-678. 
Chen, P., Murphy-Ullrich, J.E., and Wells, A. (1996). A role for gelsolin in actuating epidermal growth factor receptor-mediated cell motility. J. Cell Biol. 134, 689-698.

Chen, P., Xie, H., Sekar, M.C., Gupta, K., and Wells, A. (1994). Epidermal growth factor receptor-mediated cell motility: phospholipase $C$ activity is required, but mitogen-activated protein kinase activity is not sufficient for induced cell movement. J. Cell Biol. 127, 847-857.

Chin, H., Nakamura, N., Kamiyama, R., Miyasaka, N., Ihle, J.N., and Miura, O. (1996). Physical and functional interactions between Stat5 and the tyrosinephosphorylated receptors for erythropoietin and interleukin-3. Blood 88, 44154425 .

Chou, J., Stolz, D.B., Burke, N.A., Watkins, S.C., and Wells, A. (2002). Distribution of gelsolin and phosphoinositol 4,5-bisphosphate in lamellipodia during EGF-induced motility. Int. J. Biochem. Cell Biol. 34, 776-790.

Condeelis, J. (1993). Life at the leading edge: the formation of cell protrusions. Annu. Rev. Cell Biol. 9, 411-444.

Cooper, J.A., and Schafer, D.A. (2000). Control of actin assembly and disassembly at filament ends. Curr. Opin. Cell Biol. 12, 97-103.

Cunningham, C.C. (1995). Actin polymerization and intracellular solvent flow in cell surface blebbing. J. Cell Biol. 129, 1589-1599.

De Arruda, M.V., Bazari, H., Wallek, M., and Matsudaira, P. (1992). An actin footprint on villin. Single site substitutions in a cluster of basic residues inhibit the actin severing but not capping activity of villin. J. Biol. Chem. 267, 13079-13085.

De Corte, V., Demol, H., Goethals, M., Van Damme, J., Gettemans, J., and Vandekerckhove, J. (1999). Identification of Tyr438 as the major in vitro c-Src phosphorylation site in human gelsolin: a mass spectrometric approach. Protein Sci. 8, 234-241.

De Corte, V., Gettemans, J., and Vandekerckhove, J. (1997). Phosphatidylinositol 4,5-bisphosphate specifically stimulates PP60(c-src) catalyzed phosphorylation of gelsolin and related actin-binding proteins. FEBS Lett. 401, 191-196.

De La Cruz, E.M., Mandinova, A., Steinmetz, M.O., Stoffler, D., Aebi, U., and Pollard, T.D. (2000). Polymerization and structure of nucleotide-free actin filaments. J. Mol. Biol. 295, 517-526.

De La Cruz, E.M., and Pollard, T.D. (1995). Nucleotide-free actin: stabilization by sucrose and nucleotide binding kinetics. Biochemistry 34, 5452-5461. 
DeMali, K.A., and Burridge, K. (2003). Coupling membrane protrusion and cell adhesion. J. Cell. Sci. 116, 2389-2397.

DesMarais, V., Ghosh, M., Eddy, R., and Condeelis, J. (2005). Cofilin takes the lead. J. Cell. Sci. 118, 19-26.

Falet, H., Hoffmeister, K.M., Neujahr, R., Italiano, J.E., Jr., Stossel, T.P., Southwick, F.S., and Hartwig, J.H. (2002). Importance of free actin filament barbed ends for Arp2/3 complex function in platelets and fibroblasts. Proc. Natl. Acad. Sci. U S A 99, 16782-16787.

Ferrary, E., Cohen-Tannoudji, M., Pehau-Arnaudet, G., Lapillonne, A., Athman, R., Ruiz, T., Boulouha, L., El Marjou, F., Doye, A., Fontaine, J.J., Antony, C., Babinet, C., Louvard, D., Jaisser, F., and Robine, S. (1999). In vivo, villin is required for $\mathrm{Ca}(2+)$-dependent $\mathrm{F}$-actin disruption in intestinal brush borders. J. Cell Biol. 146, 819-830.

Finidori, J., Friederich, E., Kwiatkowski, D.J., and Louvard, D. (1992). In vivo analysis of functional domains from villin and gelsolin. J. Cell Biol. 116, 11451155.

Finlay, B.B., and Cossart, P. (1997). Exploitation of mammalian host cell functions by bacterial pathogens. Science 276, 718-725.

Folger, P.A., Berg, W.J., DeJesus, Z., Fong, Y., and Pardee, J.D. (1999). A mammalian severin replaces gelsolin in transformed epithelial cells. Cancer Res. $59,5349-5355$.

Forney, J.R., DeWald, D.B., Yang, S., Speer, C.A., and Healey, M.C. (1999). A role for host phosphoinositide 3-kinase and cytoskeletal remodeling during Cryptosporidium parvum infection. Infect. Immun. 67, 844-852.

Franck, Z., Footer, M., and Bretscher, A. (1990). Microinjection of villin into cultured cells induces rapid and long- lasting changes in cell morphology but does not inhibit cytokinesis, cell motility, or membrane ruffling. J. Cell Biol. 111, 2475-2485.

Friederich, E., Huet, C., Arpin, M., and Louvard, D. (1989). Villin induces microvilli growth and actin redistribution in transfected fibroblasts. Cell 59, 461475.

Furnish, E.J., Zhou, W., Cunningham, C.C., Kas, J.A., and Schmidt, C.E. (2001). Gelsolin overexpression enhances neurite outgrowth in PC12 cells. FEBS Lett. 508, 282-286.

George, S., Tomar, A., Wang, Y., Mathew, S., and Khurana, S. (2006). Localization of villin dimers in living cells and their potenital role in actin bundling. Submitted. 
Glenney, J.R., Jr., Bretscher, A., and Weber, K. (1980). Calcium control of the intestinal microvillus cytoskeleton: its implications for the regulation of microfilament organizations. Proc. Natl. Acad. Sci. U S A 77, 6458-6462.

Glenney, J.R., Jr., and Weber, K. (1981). Calcium control of microfilaments: uncoupling of the F-actin-severing and -bundling activity of villin by limited proteolysis in vitro. Proc. Natl. Acad. Sci. U S A 78, 2810-2814.

Golenhofen, N., Doctor, R.B., Bacallao, R., and Mandel, L.J. (1995). Actin and villin compartmentation during ATP depletion and recovery in renal cultured cells. Kidney Int. 48, 1837-1845.

Gossen, M., and Bujard, H. (1992). Tight control of gene expression in mammalian cells by tetracycline-responsive promoters. PNAS 89, 5547-5551.

Gual, P., Giordano, S., Williams, T.A., Rocchi, S., Van Obberghen, E., and Comoglio, P.M. (2000). Sustained recruitment of phospholipase C-gamma to Gab1 is required for HGF-induced branching tubulogenesis. Oncogene 19, 15091518.

Hartwig, J.H., Chambers, K.A., and Stossel, T.P. (1989). Association of gelsolin with actin filaments and cell membranes of macrophages and platelets. J. Cell Biol. 108, 467-479.

Hegyi, G., Szilagyi, L., and Belagyi, J. (1988). Influence of the bound nucleotide on the molecular dynamics of actin. Eur. J. Biochem. 175, 271-274.

Hesterberg, L.K., and Weber, K. (1983a). Demonstration of three distinct calcium-binding sites in villin, a modulator of actin assembly. J. Biol. Chem. 258, 365-369.

Hesterberg, L.K., and Weber, K. (1983b). Ligand-induced conformational changes in villin, a calcium-controlled actin-modulating protein. J. Biol. Chem. 258, 359-364.

Holmes, K.C., Popp, D., Gebhard, W., and Kabsch, W. (1990). Atomic model of the actin filament. Nature 347, 44-49.

Ichetovkin, I., Han, J., Pang, K.M., Knecht, D.A., and Condeelis, J.S. (2000). Actin filaments are severed by both native and recombinant dictyostelium cofilin but to different extents. Cell Motil. Cytoskeleton 45, 293-306.

Janmey, P.A., and Matsudaira, P.T. (1988). Functional comparison of villin and gelsolin. Effects of $\mathrm{Ca} 2+, \mathrm{KCl}$, and polyphosphoinositides. J. Biol. Chem. 263, 16738-16743.

Khurana, S., Arpin, M., Patterson, R., and Donowitz, M. (1997). lleal microvillar protein villin is tyrosine-phosphorylated and associates with PLC-gamma1. Role 
of cytoskeletal rearrangement in the carbachol-induced inhibition of ileal $\mathrm{NaCl}$ absorption. J. Biol. Chem. 272, 30115-30121.

Kim, A.S., Kakalis, L.T., Abdul-Manan, N., Liu, G.A., and Rosen, M.K. (2000). Autoinhibition and activation mechanisms of the Wiskott-Aldrich syndrome protein. Nature 404, 151-158.

Kothakota, S., Azuma, T., Reinhard, C., Klippel, A., Tang, J., Chu, K., McGarry, T.J., Kirschner, M.W., Koths, K., Kwiatkowski, D.J., and Williams, L.T. (1997). Caspase-3-generated fragment of gelsolin: effector of morphological change in apoptosis. Science 278, 294-298.

Kozlowski, M., Larose, L., Lee, F., Le, D.M., Rottapel, R., and Siminovitch, K.A. (1998). SHP-1 binds and negatively modulates the c-Kit receptor by interaction with tyrosine 569 in the c-Kit juxtamembrane domain. Mol. Cell Biol. 18, 20892099.

Kumar, N., and Khurana, S. (2004). Identification of a functional switch for actin severing by cytoskeletal proteins. J. Biol. Chem. 279, 24915-24918.

Kumar, N., Tomar, A., Parrill, A.L., and Khurana, S. (2004a). Functional dissection and molecular characterization of calcium-sensitive actin-capping and actin-depolymerizing sites in villin. J. Biol. Chem. 279, 45036-45046.

Kumar, N., Zhao, P., Tomar, A., Galea, C.A., and Khurana, S. (2004b). Association of villin with phosphatidylinositol 4,5-bisphosphate regulates the actin cytoskeleton. J. Biol. Chem. 279, 3096-3110.

Lauwaet, T., Oliveira, M.J., Callewaert, B., De Bruyne, G., Saelens, X., Ankri, S., Vandenabeele, P., Mirelman, D., Mareel, M., and Leroy, A. (2003). Proteolysis of enteric cell villin by Entamoeba histolytica cysteine proteinases. J. Biol. Chem. $278,22650-22656$.

Loisel, T.P., Boujemaa, R., Pantaloni, D., and Carlier, M.F. (1999). Reconstitution of actin-based motility of Listeria and Shigella using pure proteins. Nature 401, 613-616.

Lu, J., and Pollard, T.D. (2001). Profilin binding to poly-L-proline and actin monomers along with ability to catalyze actin nucleotide exchange is required for viability of fission yeast. Mol. Biol. Cell 12, 1161-1175.

Lu, M., Witke, W., Kwiatkowski, D.J., and Kosik, K.S. (1997). Delayed Retraction of Filopodia in Gelsolin Null Mice. J. Cell Biol. 138, 1279-1287.

Lueck, A., Brown, D., and Kwiatkowski, D.J. (1998). The actin-binding proteins adseverin and gelsolin are both highly expressed but differentially localized in kidney and intestine. J. Cell. Sci. 111, 3633-3643. 
Machesky, L.M., Atkinson, S.J., Ampe, C., Vandekerckhove, J., and Pollard, T.D. (1994). Purification of a cortical complex containing two unconventional actins from Acanthamoeba by affinity chromatography on profilin-agarose. J. Cell Biol. 127, $107-115$.

Machesky, L.M., and Hall, A. (1997). Role of actin polymerization and adhesion to extracellular matrix in Rac- and Rho-induced cytoskeletal reorganization. J. Cell Biol. 138, 913-926.

Markus, M.A., Nakayama, T., Matsudaira, P., and Wagner, G. (1994). Solution structure of villin $14 \mathrm{~T}$, a domain conserved among actin-severing proteins. Protein Sci. 3, 70-81.

Martin, P. (1997). Wound healing--aiming for perfect skin regeneration. Science 276, 75-81.

Meng, J., Vardar, D., Wang, Y., Guo, H.C., Head, J.F., and McKnight, C.J. (2005). High-resolution crystal structures of villin headpiece and mutants with reduced F-actin binding activity. Biochemistry 44, 11963-11973.

Miao, L., Vanderlinde, O., Stewart, M., and Roberts, T.M. (2003). Retraction in amoeboid cell motility powered by cytoskeletal dynamics. Science 302, 14051407.

Mogilner, A., and Oster, G. (2003). Polymer motors: pushing out the front and pulling up the back. Curr. Biol. 13, R721-733.

Mouneimne, G., Soon, L., DesMarais, V., Sidani, M., Song, X., Yip, S.C., Ghosh, M., Eddy, R., Backer, J.M., and Condeelis, J. (2004). Phospholipase C and cofilin are required for carcinoma cell directionality in response to EGF stimulation. J. Cell Biol. 166, 697-708.

Mullins, R.D., Heuser, J.A., and Pollard, T.D. (1998). The interaction of Arp2/3 complex with actin: nucleation, high affinity pointed end capping, and formation of branching networks of filaments. Proc. Natl. Acad. Sci. U S A 95, 6181-6186.

Nodder, S., and Martin, P. (1997). Wound healing in embryos: a review. Anat. Embryol. (Berl) 195, 215-228.

Nomoto, K., Tomita, N., Miyake, M., Xhu, D.B., LoGerfo, P.R., and Weinstein, I.B. (1995). Expression of phospholipases gamma 1, beta 1, and delta 1 in primary human colon carcinomas and colon carcinoma cell lines. Mol. Carcinog. 12, 146-152.

Northrop, J., Weber, A., Mooseker, M.S., Franzini-Armstrong, C., Bishop, M.F., Dubyak, G.R., Tucker, M., and Walsh, T.P. (1986a). Different calcium dependence of the capping and cutting activities of villin. J. Biol. Chem. 261, 9274-9281. 
Northrop, J., Weber, A., Mooseker, M.S., Franzini-Armstrong, C., Bishop, M.F., Dubyak, G.R., Tucker, M., and Walsh, T.P. (1986b). Different calcium dependence of the capping and cutting activities of villin. J. Biol. Chem. 261, 9274-9281.

Nusrat, A., Delp, C., and Madara, J.L. (1992). Intestinal epithelial restitution. Characterization of a cell culture model and mapping of cytoskeletal elements in migrating cells. J. Clin. Invest. 89, 1501-1511.

Panebra, A., Ma, S.X., Zhai, L.W., Wang, X.T., Rhee, S.G., and Khurana, S. (2001). Regulation of phospholipase C-gamma(1) by the actin-regulatory protein villin. Am J. Physiol. Cell Physiol. 281, C1046-1058.

Papakonstanti, E.A., Emmanouel, D.S., Gravanis, A., and Stournaras, C. (2000). PLC-gamma1 signaling pathway and villin activation are involved in actin cytoskeleton reorganization induced by $\mathrm{Na}+/ \mathrm{Pi}$ cotransport up- regulation. Mol. Med. 6, 303-318.

Park, J.G., Lee, Y.H., Kim, S.S., Park, K.J., Noh, D.Y., Ryu, S.H., and Suh, P.G. (1994). Overexpression of phospholipase C-gamma 1 in familial adenomatous polyposis. Cancer Res. 54, 2240-2244.

Parsons, J.T., and Parsons, S.J. (1997). Src family protein tyrosine kinases: cooperating with growth factor and adhesion signaling pathways. Curr. Opin. Cell Biol. 9, 187-192.

Phillips, M.J., Azuma, T., Meredith, S.L., Squire, J.A., Ackerley, C.A., Pluthero, F.G., Roberts, E.A., Superina, R.A., Levy, G.A., and Marsden, P.A. (2003). Abnormalities in villin gene expression and canalicular microvillus structure in progressive cholestatic liver disease of childhood. Lancet 362, 1112-1119.

Piccolo, E., Innominato, P.F., Mariggio, M.A., Maffucci, T., lacobelli, S., and Falasca, M. (2002). The mechanism involved in the regulation of phospholipase Cgamma1 activity in cell migration. Oncogene 21, 6520-6529.

Pinson, K.I., Dunbar, L., Samuelson, L., and Gumucio, D.L. (1998). Targeted disruption of the mouse villin gene does not impair the morphogenesis of microvilli. Dev. Dyn. 211, 109-121.

Polk, D.B. (1998). Epidermal growth factor receptor-stimulated intestinal epithelial cell migration requires phospholipase C activity. Gastroenterology 114, 493-502.

Pollard, T.D., Blanchoin, L., and Mullins, R.D. (2000). Molecular mechanisms controlling actin filament dynamics in nonmuscle cells. Annu. Rev. Biophys. Biomol. Struct. 29, 545-576. 
Pollard, T.D., and Borisy, G.G. (2003). Cellular motility driven by assembly and disassembly of actin filaments. Cell 112, 453-465.

Ponti, A., Machacek, M., Gupton, S.L., Waterman-Storer, C.M., and Danuser, G. (2004). Two distinct actin networks drive the protrusion of migrating cells. Science 305, 1782-1786.

Ponzetto, C., Bardelli, A., Zhen, Z., Maina, F., Dalla Zonca, P., Giordano, S., Graziani, A., Panayotou, G., and Comoglio, P.M. (1994). A multifunctional docking site mediates signaling and transformation by the hepatocyte growth factor/scatter factor receptor family. Cell 77, 261-271.

Pories, S.E., Hess, D.T., Swenson, K., Lotz, M., Moussa, R., Steele, G., Jr., Shibata, D., Rieger-Christ, K.M., and Summerhayes, C. (1998). Overexpression of pp60c-src elicits invasive behavior in rat colon epithelial cells. Gastroenterology 114, 1287-1295.

Reiske, H.R., Kao, S.-C., Cary, L.A., Guan, J.-L., Lai, J.-F., and Chen, H.-C. (1999). Requirement of phosphatidylinositol 3-kinase in focal adhesion kinasepromoted cell migration. J. Biol. Chem. 274, 12361-12366.

Ridley, A.J., and Hall, A. (1992). The small GTP-binding protein rho regulates the assembly of focal adhesions and actin stress fibers in response to growth factors. Cell 70, 389-399.

Rieder, G., Tessier, A.J., Qiao, X.T., Madison, B., Gumucio, D.L., and Merchant, J.L. (2005). Helicobacter-induced intestinal metaplasia in the stomach correlates with Elk-1 and serum response factor induction of villin. J. Biol. Chem. 280, 49064912.

Rohatgi, R., Ma, L., Miki, H., Lopez, M., Kirchhausen, T., Takenawa, T., and Kirschner, M.W. (1999). The interaction between N-WASP and the Arp2/3 complex links Cdc42-dependent signals to actin assembly. Cell 97, 221-231.

Rottner, K., Behrendt, B., Small, J.V., and Wehland, J. (1999). VASP dynamics during lamellipodia protrusion. Nat. Cell Biol. 1, 321-322.

Schafer, D.A., Jennings, P.B., and Cooper, J.A. (1996). Dynamics of capping protein and actin assembly in vitro: uncapping barbed ends by polyphosphoinositides. J. Cell Biol. 135, 169-179.

Singh, A.B., Tsukada, T., Zent, R., and Harris, R.C. (2004). Membraneassociated HB-EGF modulates HGF-induced cellular responses in MDCK cells. J. Cell Sci. 117, 1365-1379.

Small, J.V., Herzog, M., and Anderson, K. (1995). Actin filament organization in the fish keratocyte lamellipodium. J. Cell Biol. 129, 1275-1286. 
Smith, M.R., Court, D.W., Kim, H.K., Park, J.B., Rhee, S.G., Rhim, J.S., and Kung, H.F. (1998). Overexpression of phosphoinositide-specific phospholipase Cgamma in $\mathrm{NIH}$ 3T3 cells promotes transformation and tumorigenicity. Carcinogenesis 19, 177-185.

Soderquist, A.M., Todderud, G., and Carpenter, G. (1992). Elevated membrane association of phospholipase C-gamma 1 in MDA-468 mammary tumor cells. Cancer Res. 52, 4526-4529.

Sonnenberg, E., Meyer, D., Weidner, K.M., and Birchmeier, C. (1993). Scatter factor/hepatocyte growth factor and its receptor, the c-met tyrosine kinase, can mediate a signal exchange between mesenchyme and epithelia during mouse development. J. Cell Biol. 123, 223-235.

Strzelecka-Golaszewska, H. (2001). Divalent cations, nucleotides, and actin structure. Results Probl. Cell Differ. 32, 23-41.

Sun, H.Q., Kwiatkowska, K., Wooten, D.C., and Yin, H.L. (1995). Effects of CapG overexpression on agonist-induced motility and second messenger generation. J. Cell Biol. 129, 147-156.

Svitkina, T.M., and Borisy, G.G. (1999). Arp2/3 complex and actin depolymerizing factor/cofilin in dendritic organization and treadmilling of actin filament array in lamellipodia. J. Cell Biol. 145, 1009-1026.

Tomar, A., George, S., Kansal, P., Wang, Y., and Khurana, S. (2006). Interaction of phospholipase C-\{gamma\}1 with villin regulates epithelial cell migration. J. Biol. Chem. 281, 31972-31986.

Tomar, A., Wang, Y., Kumar, N., George, S., Ceacareanu, B., Hassid, A., Chapman, K.E., Aryal, A.M., Waters, C.M., and Khurana, S. (2004). Regulation of cell motility by tyrosine phosphorylated villin. Mol. Biol. Cell 15, 4807-4817.

Trinkaus, J.P. (1988). Directional cell movement during early development of the teleost Blennius pholis: I. Formation of epithelial cell clusters and their pattern and mechanism of movement. J. Exp. Zool. 245, 157-186.

Tsukamoto, T., Inada, K., Tanaka, H., Mizoshita, T., Mihara, M., Ushijima, T., Yamamura, Y., Nakamura, S., and Tatematsu, M. (2004). Down-regulation of a gastric transcription factor, Sox2, and ectopic expression of intestinal homeobox genes, $\mathrm{Cdx} 1$ and $\mathrm{Cdx} 2$ : inverse correlation during progression from gastric/intestinal-mixed to complete intestinal metaplasia. J. Cancer. Res. Clin. Oncol. 130, 135-145.

Villereal, M.L., and Palfrey, H.C. (1989). Intracellular calcium and cell function. Annu. Rev. Nutr. 9, 347-376. 
Walsh, T.P., Weber, A., Davis, K., Bonder, E., and Mooseker, M. (1984). Calcium dependence of villin-induced actin depolymerization. Biochemistry 23, 60996102.

Wang, Y., George, S., Tomar, A., Chatman, L., and Khurana, S.. Obligatory role for phospholipase C-g1 in epithelial cell migration (Submitted).

Waters, C.M., and Savla, U. (1999). Keratinocyte growth factor accelerates wound closure in airway epithelium during cyclic mechanical strain. J. Cell Physiol. 181, 424-432.

Wegner, A. (1976). Head to tail polymerization of actin. J. Mol. Biol. 108, 139150.

Wegner, A., and Isenberg, G. (1983). 12-fold difference between the critical monomer concentrations of the two ends of actin filaments in physiological salt conditions. Proc. Natl. Acad. Sci. U S A 80, 4922-4925.

Welch, M.D., Rosenblatt, J., Skoble, J., Portnoy, D.A., and Mitchison, T.J. (1998). Interaction of human Arp2/3 complex and the Listeria monocytogenes ActA protein in actin filament nucleation. Science 281, 105-108.

Wells, A. (2000). Tumor invasion: role of growth factor-induced cell motility. Adv. Cancer Res. 78, 31-101.

White, E., Blose, S.H., and Stillman, B.W. (1984). Nuclear envelope localization of an adenovirus tumor antigen maintains the integrity of cellular DNA. Mol. Cell. Biol. 4, 2865-2875.

White, P., Doctor, R.B., Dahl, R.H., and Chen, J. (2000). Coincident microvillar actin bundle disruption and perinuclear actin sequestration in anoxic proximal tubule. Am J. Physiol. Renal Physiol. 278, F886-893.

Winter, D., Lechler, T., and Li, R. (1999). Activation of the yeast Arp2/3 complex by Bee1p, a WASP-family protein. Curr. Biol. 9, 501-504.

Witke, W., Sharpe, A.H., Hartwig, J.H., Azuma, T., Stossel, T.P., and Kwiatkowski, D.J. (1995). Hemostatic, inflammatory, and fibroblast responses are blunted in mice lacking gelsolin. Cell 81, 41-51.

Yuan, X., and Desiderio, D.M. (2003). Proteomics analysis of phosphotyrosylproteins in human lumbar cerebrospinal fluid. J. Proteome Res. 2, 476-487.

Zhai, L., Kumar, N., Panebra, A., Zhao, P., Parrill, A.L., and Khurana, S. (2002). Regulation of actin dynamics by tyrosine phosphorylation: identification of tyrosine phosphorylation sites within the actin-severing domain of villin. Biochemistry 41, 11750-11760. 
Zhai, L., Zhao, P., Panebra, A., Guerrerio, A.L., and Khurana, S. (2001). Tyrosine phosphorylation of villin regulates the organization of the actin cytoskeleton. J. Biol. Chem. 276, 36163-36167.

Zigmond, S.H. (1996). Signal transduction and actin filament organization. Curr. Opin. Cell Biol. 8, 66-73. 


\section{Appendix A}

\section{Supplemental material for chapter $2^{3}$}

3 Permission to reproduce by The American Society for Cell Biology. Tomar, A., Wang, Y., Kumar, N., George, S., Ceacareanu, B., Hassid, A., Chapman, K.E., Aryal, A.M., Waters, C.M., and Khurana, S. (2004). Regulation of cell motility by tyrosine phosphorylated villin. Mol. Biol. Cell 15, 4807-4817. 

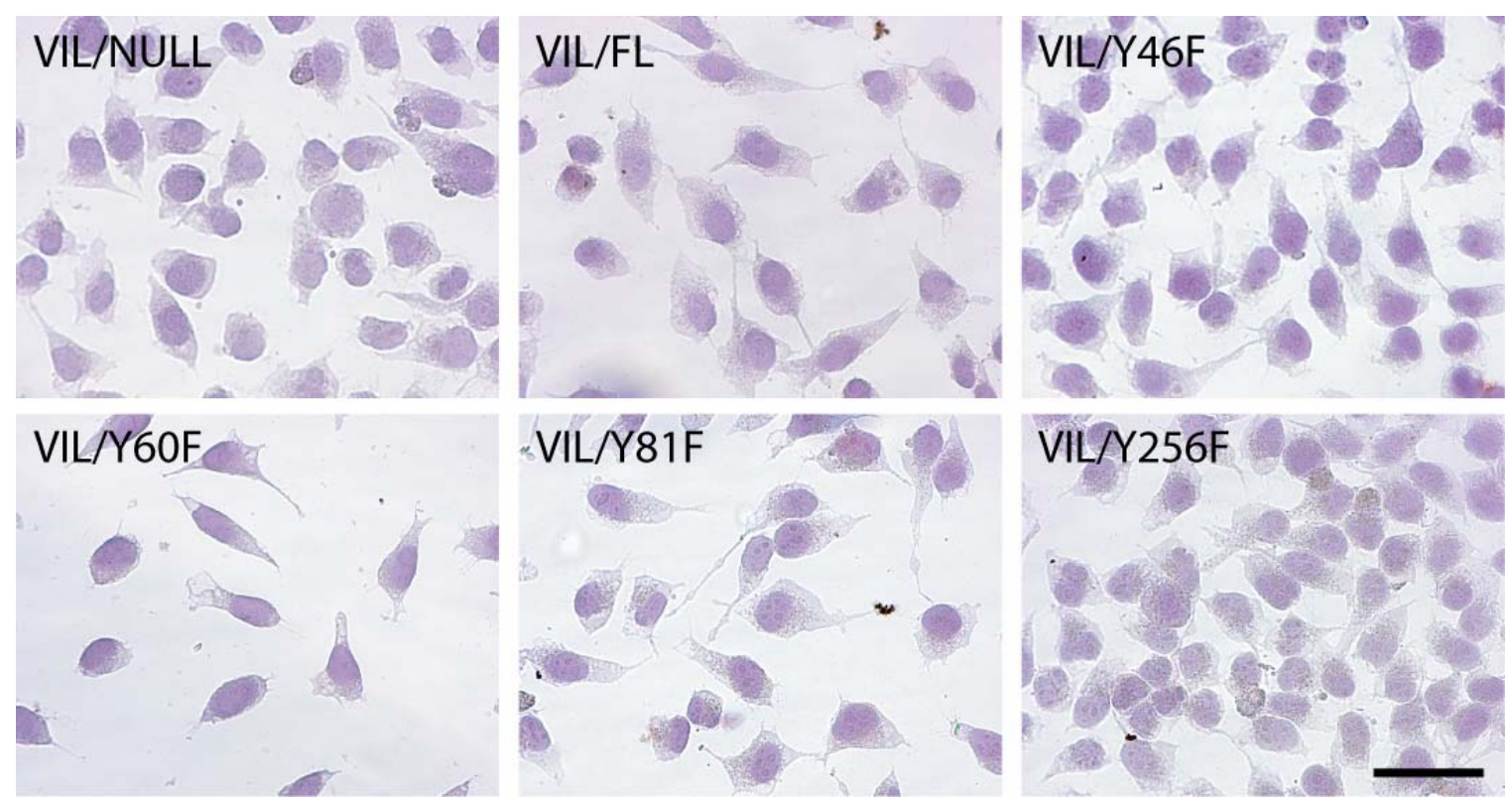

Figure A.1 There is no change in cell proliferation in HeLa cells expressing wildtype and mutant villin proteins. Cell proliferation was measured in HeLa cells stably transfected with wild-type and mutant villin proteins. Cells were cultured in low serum $(1.0 \%$ fetal bovine serum) similar to those used for cell migration studies. Cell proliferation was detected using BrdU immunostaining. Data are representative of three experiments with similar results. Bar, $50 \mu \mathrm{m}$ 


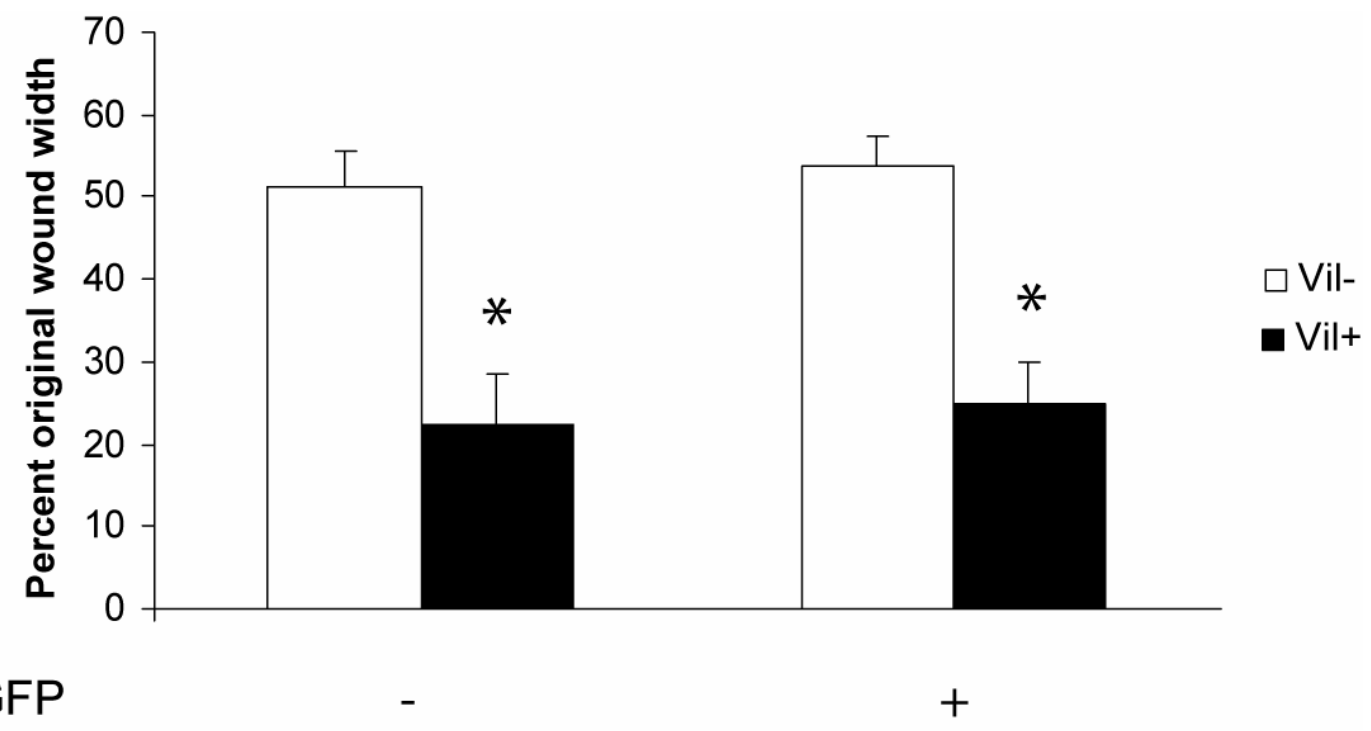

Figure A.2 There is no change in villin-induced cell migration in HeLa Tet-Off cells infected with recombinant adenovirus vector. VIL/FL and VIL/NULL cells were infected with recombinant adenovirus (Ad-EGFP (vector alone, control) for 4 hours at a multiplicity of infection (m.o.i.) of 100 . Virus-containing media was then removed and cells were cultured for an additional 18 hours to allow for expression of transgenic proteins. This was followed by measurement of cell migration. The error bars are the measured SEM and the asterisk $\left({ }^{*}\right)$ and denote statistically significant values ( $p<0.05, n=24$, compared with VIL (-) cells). 
Figure A.3 Tyrosine phosphorylation of villin is required for villin-induced increase in cell migration. HeLa cells were stably transfected with wild-type (VIL/FL) and phosphorylation site mutants of villin, namely Y46F, Y60F, Y81F and Y256F. HeLa cells transfected with wild-type (VIL/FL) and mutant villin proteins were analyzed by confocal microscopy. Double staining of villin (a2-f2) and F-actin (a1-f1) was performed using villin monoclonal antibodies (1:100) and FITC conjugated anti-mouse IgG (1:200) and Alexa Phalloidin $568(1 \mu \mathrm{g} / \mathrm{ml})$ respectively. Composite images of villin (green) and F-actin staining (red) are shown. Merged images (a3-f3) show co-localization of villin and F-actin. Wildtype villin and VIL/Y46F co-localize with F-actin at the cell periphery. In contrast phosphorylation mutants of villin VIL/Y60F, VIL/Y81F, and VIL/Y256F show intracellular distribution of villin and F-actin with minimal colocalization of villin and F-actin at the cell surface. Bars, $10 \mu \mathrm{m}$. 


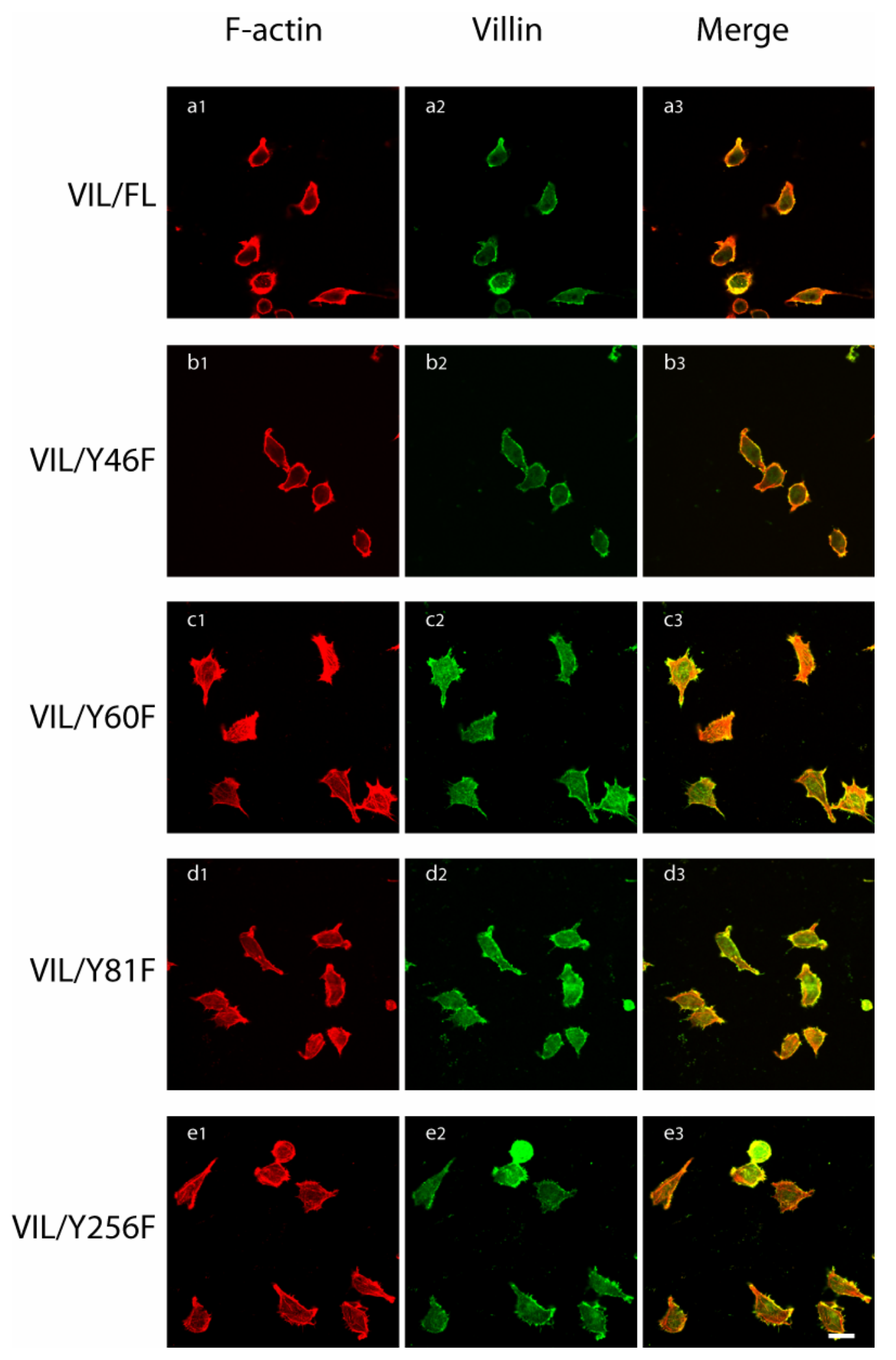




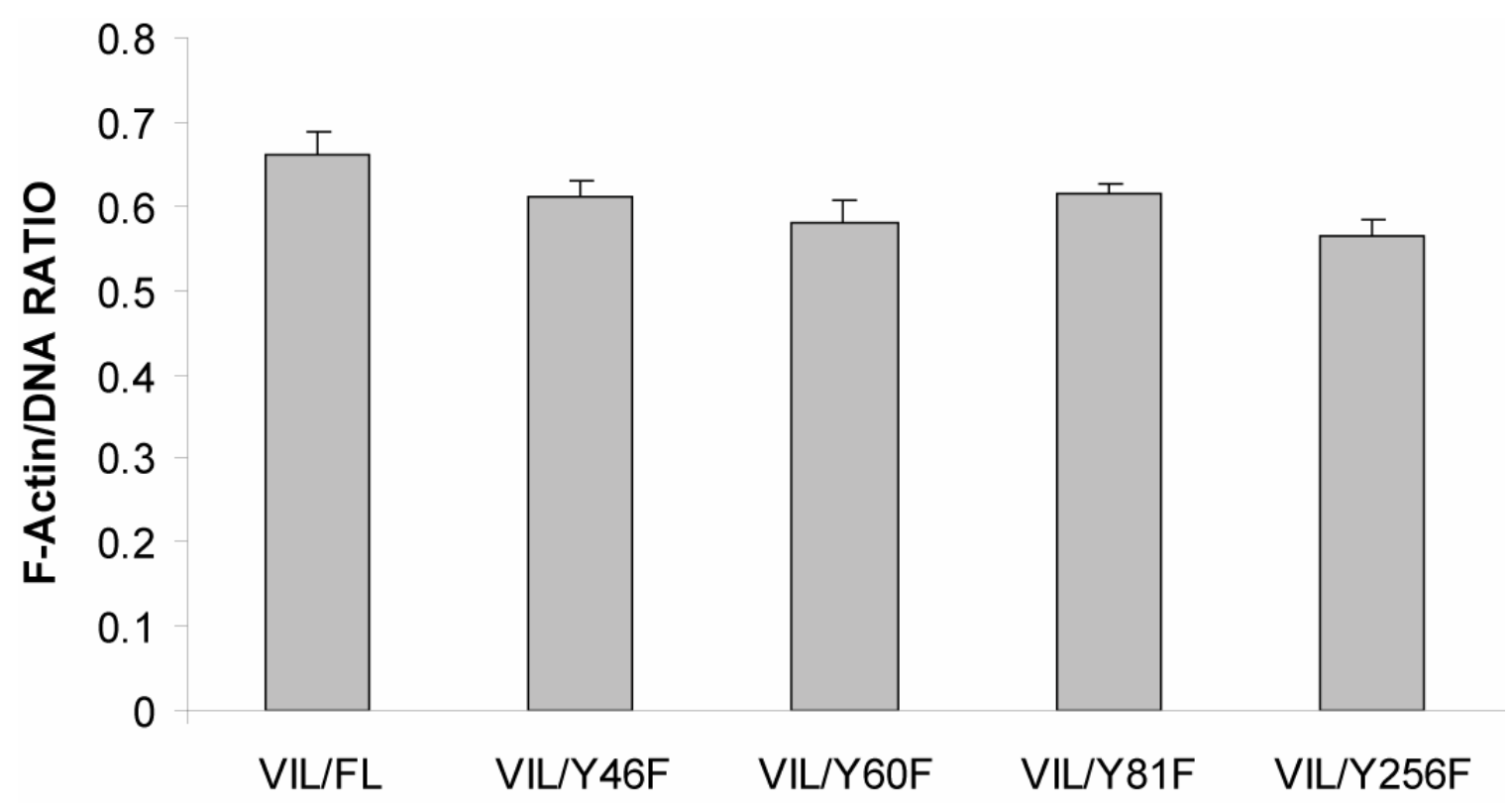

Figure A.4 Overexpression of villin does not change the F-actin content of the cells. HeLa cells expressing wild-type and mutant-villin proteins were cultured to confluence and then denuded using a pipette tip to generate 20 wounds per dish. The cells were fixed, permeabilized and stained with Alexa Phalloidin 568 and ethidium bromide. After scraping the cells in methanol, the Alexa Phalloidin and ethidium bromide were quantified using a spectrofluorometer at excitation of 525 $\mathrm{nm}$ and emission of 605 for DNA and excitation wavelength of $578 \mathrm{~nm}$ and emission of $600 \mathrm{~nm}$ for F-actin. The F-actin content was quantified as the ratio of Alexa Phalloidin fluorescence intensity to ethidium bromide fluorescence intensity. 
Figure A.5 Phosphorylation of villin at Y-60, Y-81 and Y-256 is required for villininduced cell migration. A. HeLa cells were stably transfected with wild-type (VIL/FL) and phosphorylation site mutants of villin, namely Y46E, Y60E, Y81E and Y256E. This figure shows representative clones of each villin construct transfected in HeLa cells. Data are representative of six experiments with similar results. B. Villin expression results in reorganization of the actin cytoskeleton. HeLa cells transfected with wild-type (VIL/FL) and mutant villin proteins were analyzed by confocal microscopy. Double staining of villin and F-actin was performed using villin monoclonal antibodies (1:100) and FITC conjugated antimouse IgG (1:200) and Alexa Phalloidin $568(1 \mu \mathrm{g} / \mathrm{ml})$ respectively. Composite images of villin (green) and F-actin staining (red) are shown. Merged images show co-localization of villin and F-actin (a3-f3). Wild-type villin and VIL/Y60E, VIL/Y81E, and VIL/Y256E co-localize with F-actin at the cell periphery. In contrast a phosphorylation mutant of villin VIL/Y46E shows intracellular distribution of villin and F-actin with minimal co-localization of villin and F-actin at the cell surface. Bars, $3 \mu \mathrm{m}$. C. HeLa cells expressing equal amounts of wild-type and phosphorylation site mutants ( $\mathrm{Y}$ to $\mathrm{E}$ ) of villin were used in wound-healing experiments. The error bars are the measured SEM, and the asterisk $\left({ }^{*}\right)$ and cross $(\dagger)$ denote statistically significant values $(p<0.05, n=24$, compared with VIL (-) cells) and ( $p<0.05, n=24$, compared with untreated cells) respectively. VIL (-) refers to each clone cultured in the presence of doxycycline while VIL (+) refers to the same clone cultured in the absence of doxycycline. 
F-Actin
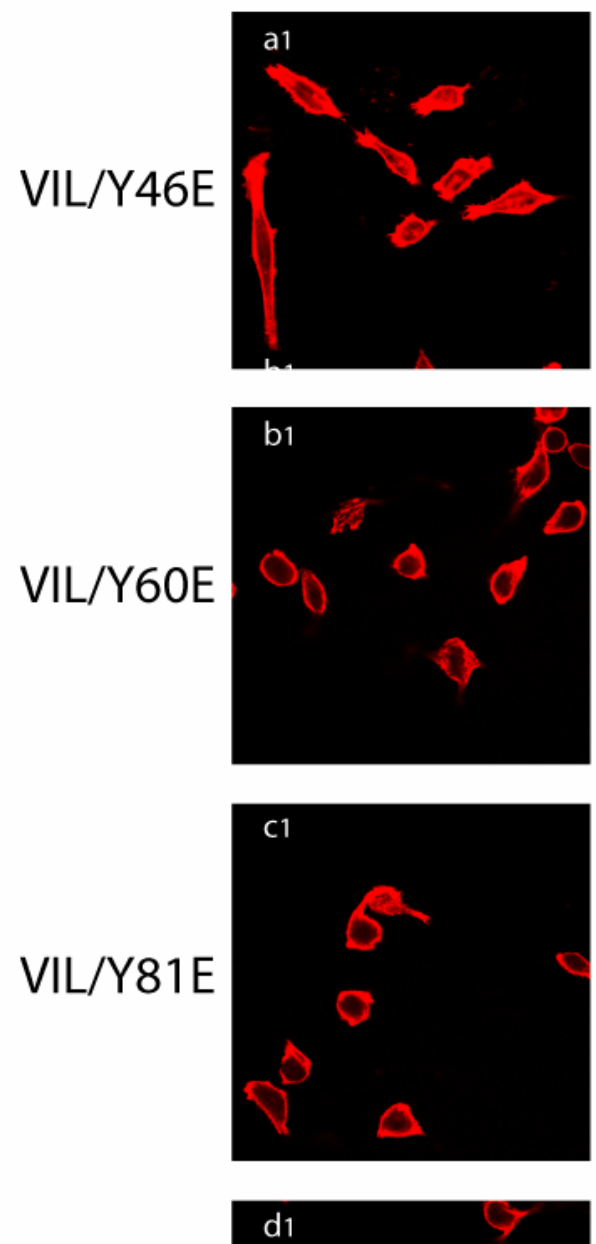

VIL/Y256E

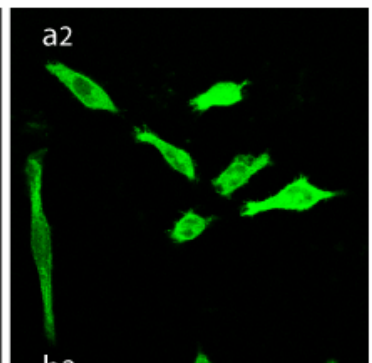

Villin
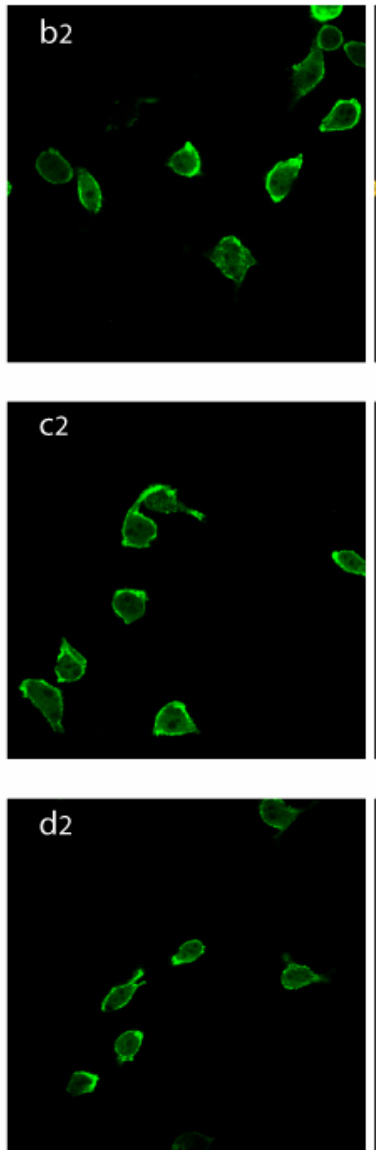

Merge
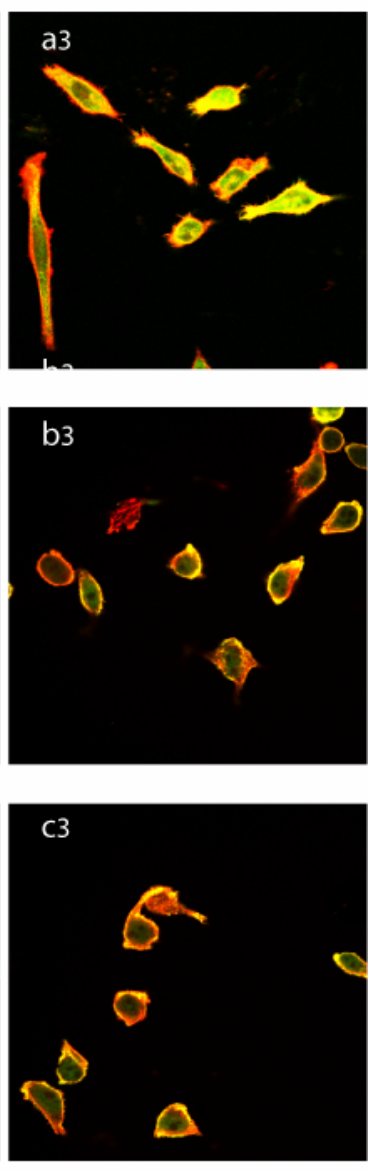

d3

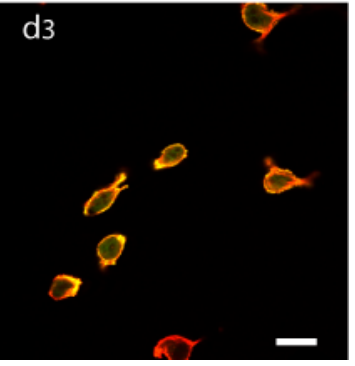




\section{Appendix B}

\section{Supplemental material for chapter $3^{4}$}

${ }^{4}$ Permission to reproduce by The American Society for Biochemistry and Molecular Biology. Tomar, A., George, S., Kansal, P., Wang, Y., and Khurana, S. (2006). Interaction of phospholipase $C_{-\gamma_{1}}$ with villin regulates epithelial cell migration. J. Biol. Chem. 281, 31972-31986. 
A.

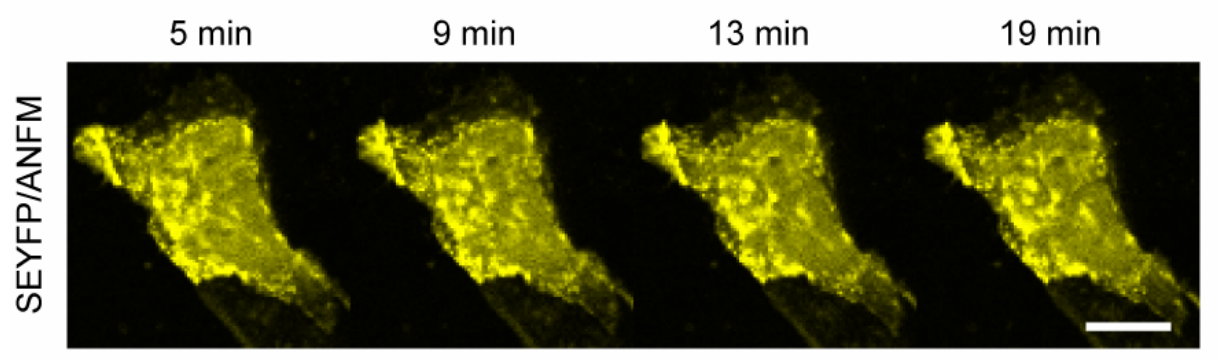

B.

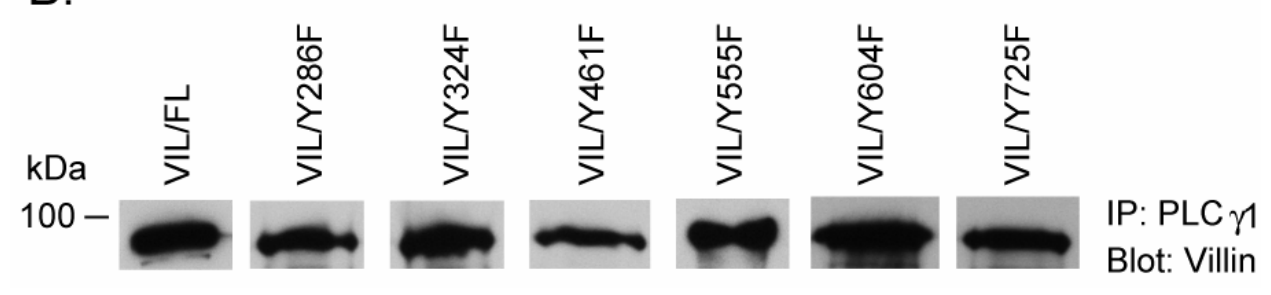

Figure B.1 Individually, the carboxyl-terminal tyrosine phosphorylation sites mutants are not associated with PLC- $\gamma_{1}$. (A) MDCK Tet-Off cells transfected with SEYFP-tagged amino-terminal phosphorylation site mutant (SEYFP/ANFM) were treated with EGF $(50 \mathrm{ng} / \mathrm{ml})$ and time-lapse images recorded 5 min after the addition of EGF for a total of $35 \mathrm{~min}$. These cells do not form lamellipodia or membrane ruffles in response to EGF treatment. (B) Triton-X-100 soluble cell extracts from HeLa Tet-Off cells stably transfected with wild-type (VIL/FL) and phosphorylation site point mutants of villin, namely VIL/Y286F; VIL/Y324F; VIL/Y461F; VIL/Y555; VIL/Y604F and VIL/Y725F were used for coimmunoprecipitation of villin with PLC- $\gamma_{1}$. These data show that VIL/FL as well as the phosphorylation site point mutants of villin associate with PLC- $\gamma_{1}$. 
Figure B.2 S1-S3 fragment of villin is pro-apoptotic. (A) HeLa Tet-Off cells transiently transfected with SEYFP/S1-S3. Images were collected 16, 20 and 24 hours post-transfection using a confocal microscope, LSM 5 PASCAL (Carl Zeiss, Thornwood, NY). At $16 \mathrm{~h}$ post-transfection cells demonstrate good expression of the S1-S3 fragment of human villin. Unlike full-length villin, this truncation mutant shows diffuse cytosolic localization. At $20 \mathrm{~h}$ post-transfection some of the cells are rounded-up (arrow head); and at $24 \mathrm{~h}$ post-transfection almost all the cells expressing S1-S3 are rounded, detached and there is a significant loss of fluorescence indicating increased cell death. (B) MDCK TetOff cells transiently transfected with SEYFP/S1-S3 were incubated with HOECHST 33258 as described in materials and methods. Apoptotic cells were distinguished from viable cells by nuclear condensation and DNA fragmentation seen as bright blue fluorescence in the nuclei indicated by arrow heads. The apoptotic cells showing bright blue fluorescence also express SEYFP/S1-S3 and the bright field image indicates that the SEYFP/S1-S3 expressing cells also show more rounded morphology. (C) MDCK Tet-Off cells transiently transfected with VIL/S1-S3 cDNA cloned in pTRE HA or vector pTRE HA alone (Control) were analyzed for apoptotic-induced DNA fragmentation using a colorimetric ELISA kit as described in methods, SEM $(p<0.01, n=6)$. 
A.

SEYFP/S1-S3

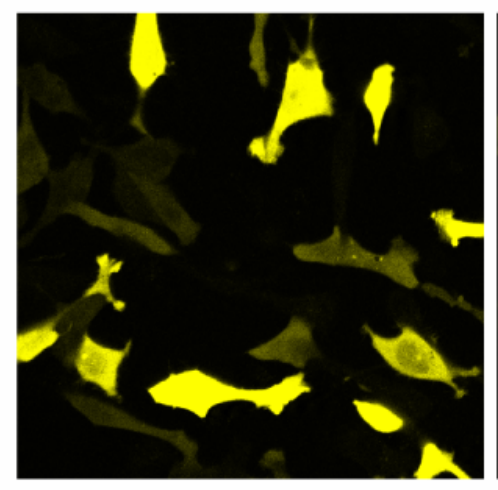

$16 \mathrm{~h}$

B.
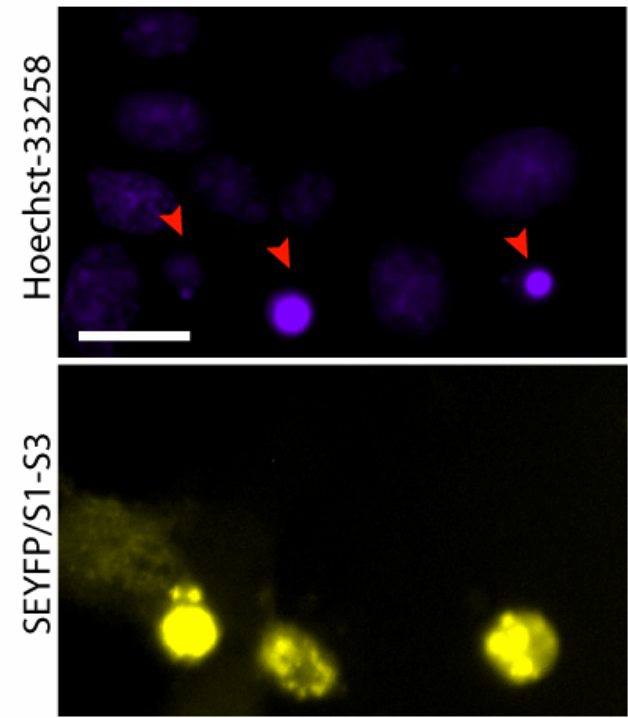

$\frac{0}{0}$
$\frac{0}{1}$
$\frac{1}{5}$
$\frac{5}{5}$
$\frac{0}{0}$
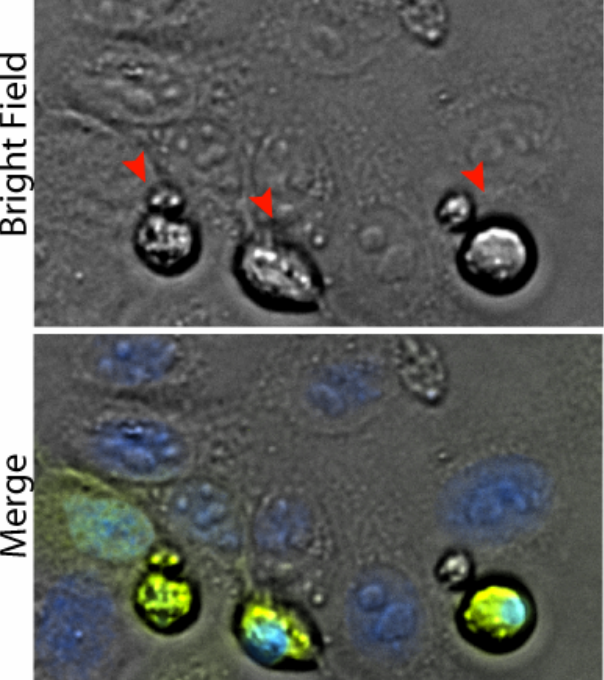

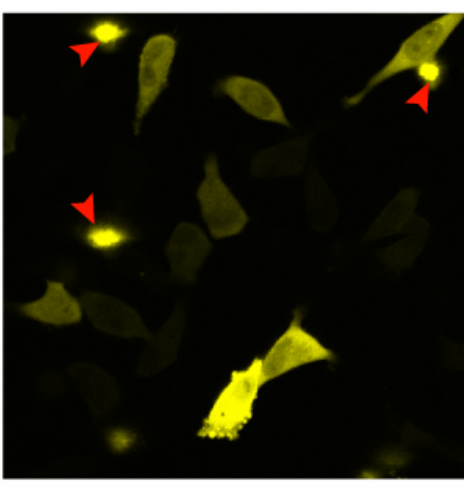

$20 \mathrm{~h}$

C.

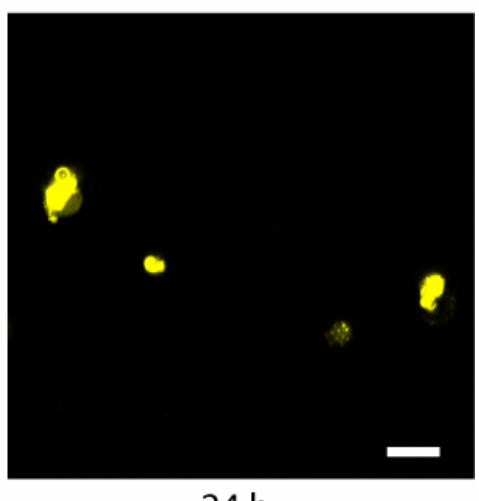

$24 \mathrm{~h}$

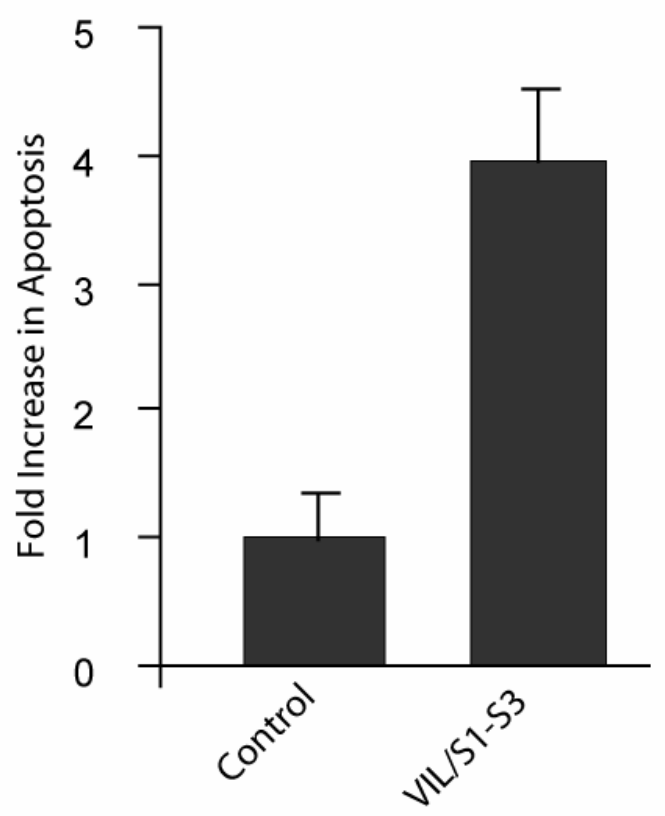




\section{Appendix C}

Glossary 


$\begin{array}{ll}\text { A } & \text { Alanine } \\ \text { a.a. } & \text { amino acid (human unless otherwise stated) } \\ \text { Ad } & \text { Adenovirus } \\ \text { Blot } & \text { Immunoblotting } \\ \text { BrdU } & \text { Bromodeoxyuridine } \\ \text { Ca }^{2+} & \text { Calcium } \\ \text { Cyt D } & \text { Cytochalasin D } \\ \text { DAG } & \text { Diacyl glycerol } \\ \text { DN } & \text { Dominant negative } \\ \text { E } & \text { Glutamic acid } \\ \text { EGF } & \text { Epidermal growth factor } \\ \text { ER } & \text { Endoplasmic reticulum } \\ \text { F } & \text { Phenylalanine } \\ \text { HGF } & \text { Hepatocyte growth factor } \\ \text { IAA } & \text { 3- } \beta \text {-indoleacrylic acid } \\ \text { IP } & \text { Immunoprecipitation } \\ \text { IP3 } & \text { Inositide tri phosphate } \\ \text { IPTG } & \text { Isopropyl } \beta \text {-D-thiogalactopyranoside } \\ \text { K } & \text { Lysine } \\ \text { Kd } & \text { Dissociation constant } \\ \text { kDa } & \text { Kilo Dalton } \\ \text { LPA } & \text { Lysophosphatidic acid } \\ \text { Mg }{ }^{2+} & \text { Magnesium } \\ \text { PI } & \text { Phosphatidylinositol } \\ \text { PIP } & \text { Phosphatidylinositol 4-monophosphate } \\ \text { PIP } 2 & \text { Phosphatidylinositol 4,5-bisphosphate } \\ \text { PKC } & \text { Protein kinase C } \\ \text { PLC- }-\gamma_{1} & \text { Phospholipase C-gamma-1 } \\ \text { PP2 } & \text { 4-amino-5-(4-chlorophenyl)-7-(t-butyl)pyrazolo(3,4-d) pyrimidine } \\ \text { PP3 } & \text { 4-amino-7-phenylpyrazolo(3,4-d)pyrimidine } \\ \text { R } & \text { Arginine } \\ \text { SEYFP } & \text { Super enhanced yellow fluorescent protein } \\ \text { VIL/ACFM } & \text { Villin mutant lacking all six carboxyl-terminal phosphorylation sites } \\ \text { VIL/ANFM } & \text { Villin mutant lacking all four amino-terminal phosphorylation sites } \\ \text { VIL/AYFM } & \text { Villin mutant lacking all ten tyrosine phosphorylation sites } \\ \text { VIL/FL } & \text { Full-length human villin } \\ \text { VIL/WT } & \text { Full-length recombinant human villin; } \\ \text { VILT/WT } & \text { Full-length recombinant human tyrosine-phosphorylated villin } \\ \text { X } & \text { Any amino acid } \\ \text { Y } & \text { Tyrosine } \\ & \\ & \end{array}$




\section{Vita}

Alok Tomar was born in Bombay, India, on April 10, 1973. His family then moved to Baroda, Gujarat where he completed his elementary school. Alok attended Maharaja Sayajirao University of Baroda, where he obtained his Bachelors of Science degree in Zoology and Master of Science degree in Biochemistry. After getting his Master of Science degree in 1997 he worked as a Diagnostic Specialist for E.Merck India Ltd., for three years. He came to University of Memphis in August 2000 as a graduate student. He later joined the lab of Dr. Seema Khurana in Department of Physiology at University of Tennessee in August 2002 to pursue his Ph.D degree. After graduating in December 2006, Alok plans to continue pursuing his research interest in cell migration and avail himself to any opportunity that allows him to contribute to this rapidly advancing field. 